\title{
Cellular, Wide-Area, and Non-Terrestrial IoT: A Survey on 5G Advances and the Road Towards 6G
}

\author{
Mojtaba Vaezi, Senior Member, IEEE, Amin Azari, Member, IEEE, Saeed R. Khosravirad, Member, IEEE, \\ Mahyar Shirvanimoghaddam, Senior Member, IEEE, M. Mahdi Azari, Member, IEEE, \\ Danai Chasaki, Senior Member, IEEE, and Petar Popovski, Fellow, IEEE
}

\begin{abstract}
The next wave of wireless technologies is proliferating in connecting things among themselves as well as to humans. In the era of the Internet of things (IoT), billions of sensors, machines, vehicles, drones, and robots will be connected, making the world around us smarter. The IoT will encompass devices that must wirelessly communicate a diverse set of data gathered from the environment for myriad new applications. The ultimate goal is to extract insights from this data and develop solutions that improve quality of life and generate new revenue. Providing large-scale, long-lasting, reliable, and near real-time connectivity is the major challenge in enabling a smart connected world. This paper provides a comprehensive survey on existing and emerging communication solutions for serving IoT applications in the context of cellular, wide-area, as well as non-terrestrial networks. Specifically, wireless technology enhancements for providing IoT access in fifth-generation (5G) and beyond cellular networks, and communication networks over the unlicensed spectrum are presented. Aligned with the main key performance indicators of $5 \mathrm{G}$ and beyond $5 \mathrm{G}$ networks, we investigate solutions and standards that enable energy efficiency, reliability, low latency, and scalability (connection density) of current and future IoT networks. The solutions include grant-free access and channel coding for short-packet communications, nonorthogonal multiple access, and on-device intelligence. Further, a vision of new paradigm shifts in communication networks in the 2030s is provided, and the integration of the associated new technologies like artificial intelligence, non-terrestrial networks, and new spectra is elaborated. In particular, the potential of using emerging deep learning and federated learning techniques for enhancing the efficiency and security of IoT communication are discussed, and their promises and challenges are introduced. Finally, future research directions toward beyond 5G IoT networks are pointed out.
\end{abstract}

Index Terms-IoT, IIoT, 5G, 6G, SigFox, LoRa, LTE-M, NBIoT, security, reliability, survival time, service availability, energyefficiency, blockchain, SDN, non-terrestrial, satellite, UAV, 3D, NOMA, random access, grant-free access, turbo code, LDPC, polar, deep leaning, federated learning.

\section{INTRODUCTION}

M. Vaezi is with the Department of Electrical and Computer Engineering, Villanova University, Villanova, PA, USA (e-mail: mvaezi@ villanova.edu).

A. Azari is with the Ericsson Research, Ericsson AB, Sweden (e-mail: amin.azari@ericsson.com).

S. R. Khosravirad is with the Nokia Bell Labs, Murray Hill, NJ, USA (e-mail: saeed.khosravirad@nokia-bell-labs.com).

M. Shirvanimoghaddam is with the School of Electrical and Information Engineering, The University of Sydney, Australia (e-mail: mahyar.shm@sydney.edu.au).

M. M. Azari is with Interdisciplinary Centre for Security, Reliability and Trust (SnT), University of Luxembourg, Luxembourg (e-mail: mohammadmahdi.azari@uni.lu)
With over 20 billion connected devices in 2020, the Internet of things (IoT) is transforming the business and consumer world in an unforeseen manner and is driving a new industrial revolution. The term IoT was coined by Kevin Ashton at MIT's Auto-ID Center in 1999 [1] to define a network that not only connects people but also 'things or objects' around them. By 2009 there were more things or objects connected to the Internet than people [1]. Today, the IoT is a huge network of things-from simple sensors to smartphones, wearables, autonomous cars, and drones-which are connected together and are used to collect and analyze data to perform actions. Although the forecasts by different analysts and consulting agencies for IoT market volume vary a lot, these all agree that IoT market volume is immense and is growing at a significant rate. According to IoT Analytics [2], by 2025 there will be more than 30 billion active IoT devices-smartphones are not included in this statistic. Business Insider forecasts that there will be more than 64 billion IoT devices by 2025 [3]. The IoT market is expected to reach $1,854.76$ billion US dollars in 2028 compared to 381.3 billion US dollars in 2021 at a compound annual growth rate compound annual growth rate (CAGR) of 25.4\% [4].

The IoT is about networks, devices, and data. It embodies the communication and interaction between objects and embedded devices from diverse environments to collect data, process it, and leverage resulting information and knowledge to make the world around us better via smarter and/or automated decision-making. To progress toward this goal, IoT devices, data, and networks are intertwined.

IoT devices and their associated hardware are very diverse and include a wide range: low-cost sensing modules (e.g., temperature, light, pressure, humidity, proximity sensors) used in smart homes, industry, agriculture, logistics, retails, etc.; wearable electronic devices worn on different parts of the body (e.g., glasses, helmets, collars, watches, wristbands, socks, shoes, etc.); more powerful devices such as automation actuators, robot arms, drones, driver-less cars, and so on; as well as desktops, tablets, and cellphones which are integral parts of IoT today. The diversity of devices and associated applications results in heterogeneous data in various senses.

The data generated by IoT devices is large-scale streaming

D. Chasaki is with the Department of ECE, Villanova University, Villanova, PA, USA (e-mail: danai.chasaki@villanova.edu).

Petar Popovski is with the Department of Electronic Systems, Aalborg University, Denmark (e-mail: petarp@es.aau.dk). 


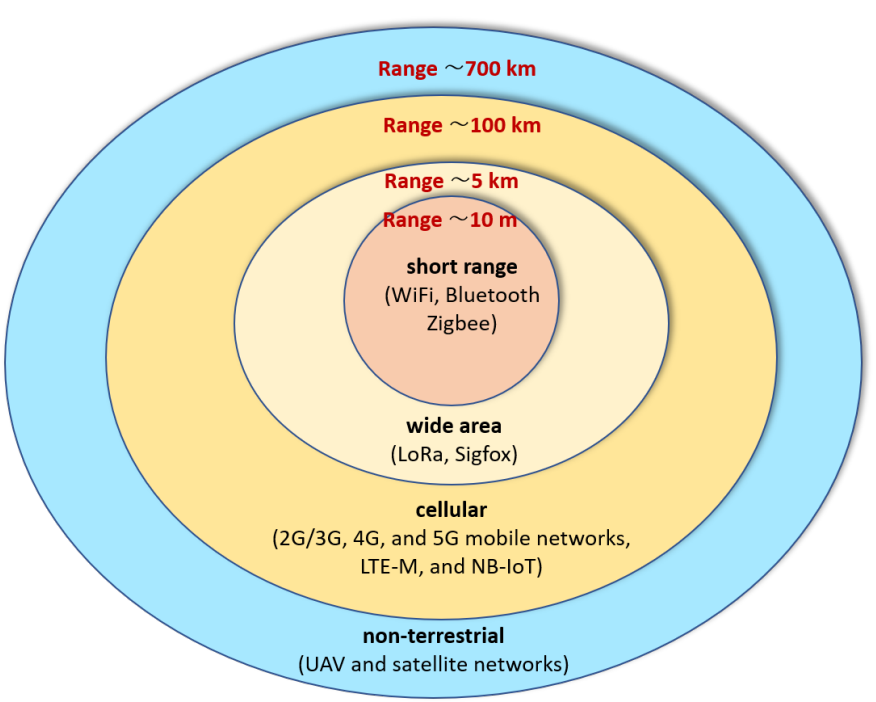

Fig. 1: Different types of IoT networks in terms of range (nonterrestrial, cellular, wide-area, and short-range), their approximate coverage range, and main representative protocols.

data that is heterogeneous, highly noisy, and highly correlated in space and time. IoT data is classified according to $6 \mathrm{Vs}$ : volume (large quantity), velocity (high rate of data production), variety (different types of data, e.g., text, audio, video, sensory data), variability (constantly changing), veracity (level of confidence or trust in the data), and value (valuable information and business insights out of it) [5]. To extract valuable information and insights, such diverse data is typically transmitted to the cloud or fusion centers via wireless networks.

Wireless access networks are crucial for the widespread adoption of IoT devices. IoT technologies have been extensively studied and classified in recent years. A widely accepted classification is in terms of coverage range which divides IoT networks into cellular, wide-area, and short-range IoT [6]. Non-terrestrial networks (NTNs) represent a new category that provides extremely wide-area and three-dimensional (3D) coverage. ${ }^{1}$ NTNs can be seen as a generalization of cellular and wide-area networks. The approximate range of networks, along with some well-known examples of them, are illustrated in Fig. 1. Cellular and wide-area IoT are the fastest-growing types. Ericsson mobility report projects CAGRs of $23 \%$ and $22 \%$ for these IoT types, between 2020 and 2026 [6]. This will challenge the incumbent and emerging cellular networks in various senses.

This survey is mainly about cellular, wide-area, and nonterrestrial IoT, their applications and challenges. To better understand the challenges and requirements, we first elaborate on existing access networks and main IoT applications in the following.

\section{A. Application Types}

In terms of applications (required data rate, latency, etc.), we can have two very different types of IoT: massive IoT and

\footnotetext{
${ }^{1}$ The terms NTNs and 3D networks are used interchangeably in this paper and refer to both unmanned aerial vehicle (UAV)-based and satellite-based systems.
}

critical IoT. There are also two other important forms of IoT, namely, broadband and industrial IoT, as shown in Fig. 2 [7]. These four IoT segments are briefly described in the following.

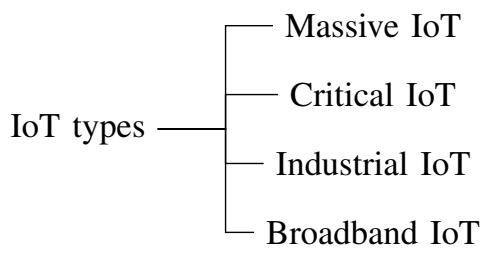

Massive IoT, a.k.a. massive machine type of communication (mMTC), describes applications with data collection purpose where a massive number of low-power endpoints (e.g., lowcost sensors) continuously and infrequently (mostly sporadically) transmit small volume of data to the cloud or fusion centers. Examples of massive IoT are the sensors used to read the temperature, pressure, light, etc., in smart homes/buildings, smart meters, and smart agriculture. In this case, transmitted data has a low volume but the number of IoT devices is very large. Massive IoT devices are usually expected to work for up to 10 years without a change of battery. Long Term Evolution (LTE) networks already support massive IoT via narrowband IoT (NB-IoT)/LTE-MTC (LTE-M).

Critical IoT, in contrast, involves fewer endpoints handling large volumes of data. IoT devices in this category go beyond just data collection and represent high-bandwidth and lowlatency applications, technically known as ultra-reliable lowlatency communications (URLLC). Industrial control, robotic machines and autonomous vehicles are examples of critical IoT, all of which require real-time data transmission. These two types of IoT applications have very different requirements, as shown in Fig. 2.

Industrial IoT (IIoT) connects people, data, and machines in the world of manufacturing and its virtual digital twin. The IIoT is a key enabler of Industry 4.0 [8] which refers to the fourth phase in the industrial revolution-the previous three being mechanical production (1784), mass production (1870), and then automation via electronics/computers (1970) [9]. Industry 4.0 is a digital transformation that focuses heavily on exploiting inter-connectivity, automation, and real-time data. IIoT, artificial intelligence (AI), big data, cloud infrastructure, and cybersecurity are the key technologies working together to realize this transformation [10]. The data created by the IIoT can be leveraged into optimizing efficiencies across the manufacturing operation from supply to distribution and aftersale services. The most noteworthy benefits manufacturers can expect from Industry 4.0 are optimized processes, higher asset utilization, greater labor productivity, better insight into the entire supply chain and production process, and after-sale services.

Broadband IoT brings mobile broadband connectivity to IoT devices, providing lower latency and higher throughput than the mMTC. Typical applications are advanced wearables, drones, augmented reality, and virtual reality devices and sensors that require greater capabilities than LTE-M or NB-IoT can provide [7]. LTE-capable smartwatches and LTE networks already supporting broadband IoT. LTE-connected drones two examples of such device categories. Fifth-generation (5G) 

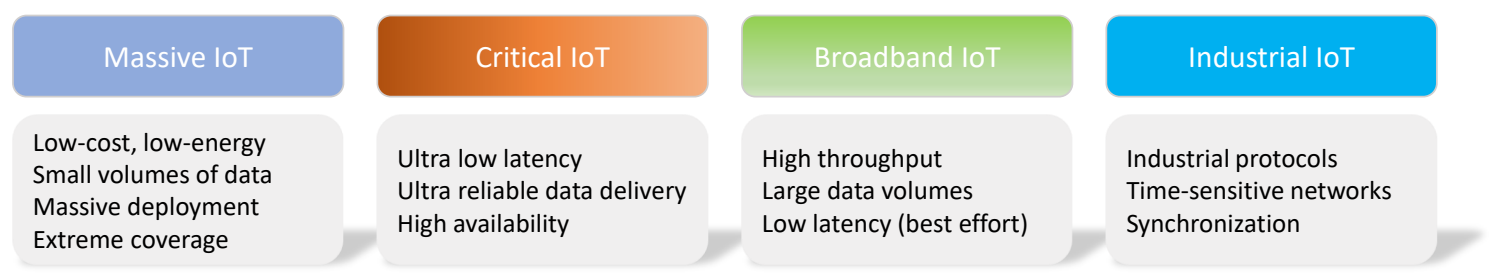

Fig. 2: Different IoT segments and their characteristics [7].

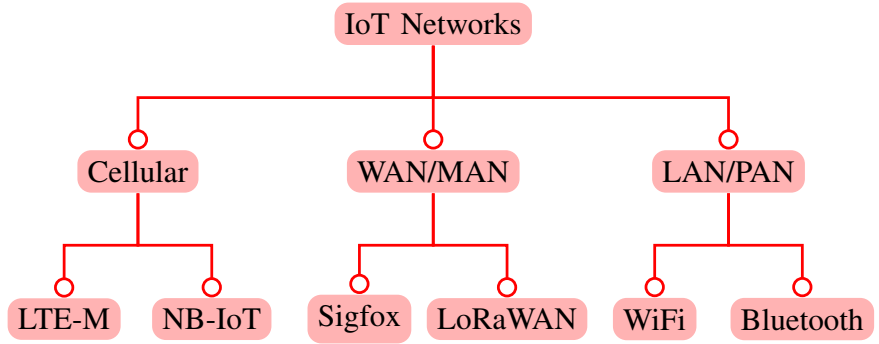

Fig. 3: IoT access network types some representative wireless protocols within each category.

cellular networks will boost broadband IoT performance.

\section{B. Access Networks}

A reliable connection between devices, sensors, and IoT platform is a key for any successful IoT project. IoT wireless networks must support a wide variety of connected devices and applications, as described earlier. Such a broad diversity makes it difficult, if not impossible, to have one technology that fits every situation. Choosing the right network requires consideration of various factors from coverage, power consumption, battery size, and deployment cost. Hence, we have to make a trade-off somewhere by choosing any of these wireless technologies.

IoT wireless connectivity and technologies can be classified in several ways, e.g., in terms of coverage range (short, medium, long, or 3D), spectrum (licensed or unlicensed), data rates (low or high), technologies (cellular or non-cellular), power requirements (low or high), standard (3rd generation partnership project (3GPP) or non-3GPP), cost (low or high), etc. A common classification is to have cellular IoT, widearea IoT, and short-range IoT [6], which respectively have long, medium, and short ranges. Within each category, there may be several wireless protocols as depicted in Fig. 3. In particular, the cellular IoT standards can be divided as

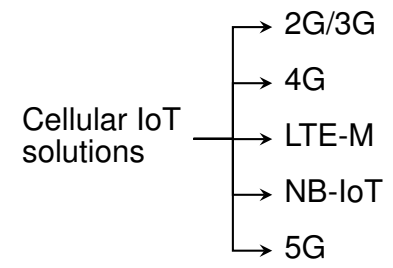

In general, IoT devices may access any of the above cellular networks including the legacy second-generation (2G) and third-generation $(3 \mathrm{G})$ cellular networks. These networks still play an important role and accommodate many IoT devices. Specifically, nearly one billion IoT devices are still connected to the legacy $2 \mathrm{G} / 3 \mathrm{G}$ networks (see [6, Fig. 14]) and, according to [11], this number will increase. Also, an exponentially increasing number of broadband IoT devices are being connected to the incumbent fourth-generation (4G) networks. However, the above solutions are not designed for massive IoT devices, which are low power modules and need extended coverage.

To handle such use cases, new solutions like NB-IoT and LTE-M have been introduced in LTE networks. NB-IoT was standardized in the 3GPP LTE Release 13 in 2016 to provide wide-area connectivity for mMTC [12]. In LTE Release 14, NB-IoT was further developed to deliver an enhanced user experience [13]. ${ }^{2}$ NB-IoT supports major mMTC requirements, such as a long battery lifetime and a massive number of devices in a cell, and extends coverage beyond existing cellular technologies.

Unlike 4G networks which were primarily designed with MMB applications in mind, and IoT solutions like NB-IoT came in later, $5 \mathrm{G}$ wireless access is intended not just to evolve mobile broadband but to be a key IoT enabler. Specifically, 5G wireless access has been developed with three broad use cases in mind [15]:

- enhanced mobile broadband (eMBB)

- massive machine-type communications (mMTC)

- ultra-reliable low-latency communications (URLLC)

The IoT segments in Fig. 2 can be related to these uses cases as follows: mMTC represents massive, eMBB represents broadband IoT, and URLLC may represent both critical and industrial IoT. Besides, there are various key performance indicator (KPI) requirements for 5G cellular networks as listed in Table I [16]. Each KPI is related to one or more use cases. The KPIs on mMTC and URLLC are specifically related to IoT. It is worth mentioning that, despite challenging requirements, only a subset of Industry 4.0 use cases are addressable by current 5G KPIs, and sixth-generation (6G) still has much work to do.

\section{C. $6 G$ Vision and Requirements}

Although industry and standardization bodies are still working around 5G mobile technology, and 5G commercialization is still in its initial stages, many researchers in academia and industry have already started asking what is next. international

\footnotetext{
${ }^{2}$ The main difference between NB-IoT and legacy LTE is in the uplink design. In NB-IoT the subcarrier spacing can be $3.75 \mathrm{kHz}$ as well as the 15 $\mathrm{kHz}$ used in legacy LTE systems [14].
} 
TABLE I: 5G key performance indicators

\begin{tabular}{lcc} 
KPI & Requirement & Category \\
\hline Peak data rate & $20 \mathrm{Gbps}$ (downlink), 10 Gbps (uplink) & eMMB \\
User experienced data rate & $100 \mathrm{Mbps}$ (downlink), 50 Mbps (uplink) & eMMB \\
Spectral efficiency & $30 \mathrm{bit} / \mathrm{s} / \mathrm{Hz}$ (downlink), $15 \mathrm{bit} / \mathrm{s} / \mathrm{Hz}$ (uplink) & eMMB \\
Latency (user plane) & $4 \mathrm{~ms}$ for eMBB, $1 \mathrm{~ms}$ for URLLC & eMMB, URLLC \\
Reliability & $1-10^{-5}$ & URLLC \\
Energy efficiency & Qualitative & eMMB \\
Connection density & $10^{6}$ devices $/ \mathrm{Km}^{2}$ & mMTC \\
Mobility & up to $500 \mathrm{Km} / \mathrm{h}$ & eMMB \\
\hline
\end{tabular}

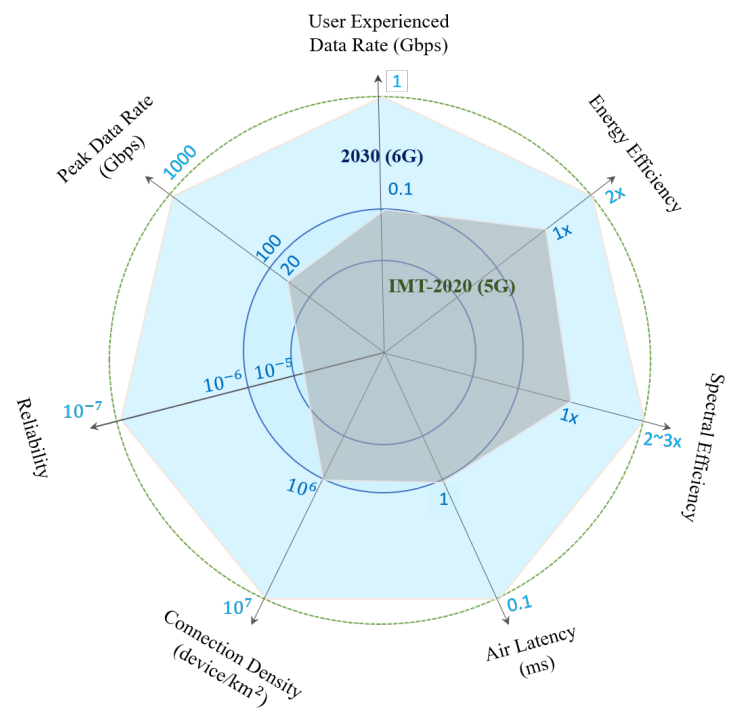

Fig. 4: Expected key performance specifications and requirements in the 2030s versus 5G (IMT-2020)

mobile telecommunications (IMT) ${ }^{3}$ plans to complete its $6 \mathrm{G}$ vision by 2023 and to develop technical requirements for $6 \mathrm{G}$ via industry standards organizations, such as the $3 \mathrm{GPP}$.

1) Technical requirements: Broadly speaking, the goal of $6 \mathrm{G}$ wireless networks is to move 5G forward another order of magnitude on various technical parameters like throughput, number of connections, latency, and reliability. 6G is also expected to make spectral and energy efficiency even greater than $5 \mathrm{G}$, to allow new connected services such as networkconnected drones, tactile Internet, real-time augmented reality glasses, etc., and to support mobility exceeding $1000 \mathrm{~km} / \mathrm{h}$ for flying objects (e.g., airplanes) [17, 18].

Figure 4 illustrates the aspired requirement of wireless networks in the 2030s, or loosely speaking $6 \mathrm{G}$ networks, based on the ITU. The requirements are also compared with those of 5G networks, i.e., IMT-2020. Other aspects of networks in the 2030s, such as architecture, new use cases, evaluation methodology, are under study [19].

${ }^{3}$ IMT is the generic term used by the International Telecommunications Union (ITU) community to designate mobile broadband systems. IMT standards are specifications and requirements for mobile broadband service that fulfill established technical parameters such as peak data rate, latency, reliability, spectrum efficiency, and so forth.
2) Vision: 6G is envisioned to bring new paradigm shifts to wireless access networks in terms of coverage, spectrum, applications, and security [17].

- 3D coverage: In the 2030s, NTNs such as UAVs [20,21] and satellite communication networks will complement the current terrestrial. This will result in a global 3D communication network overage in the ground, sea, and space.

- AI-integrated: AI and machine learning have the potential to disrupt the design of future wireless networks. It has already penetrated network technologies and will be ubiquitous across all domains (core, access, edge, device), will impact the design of 6G networks, and will proliferate new smart applications. Particularly, big data technologies combined with deep learning will improve network management, automation, and efficiency.

- All frequencies: While $5 \mathrm{G}$ is based on the radio frequency (RF) bands, i.e., sub-6 GHz and millimeter-wave (mmWave) bands (spectrum between $30 \mathrm{GHz}$ and 300 $\mathrm{GHz}$ ), new frequency bands like terahertz band $(0.1-10$ terahertz (THz)) [22] and optical frequency band [23, 24] will be exploited in 6G. Moving into higher frequency bands is a key trend for achieving 1000 Gbps peak data rates and for driving more values from the spectrum.

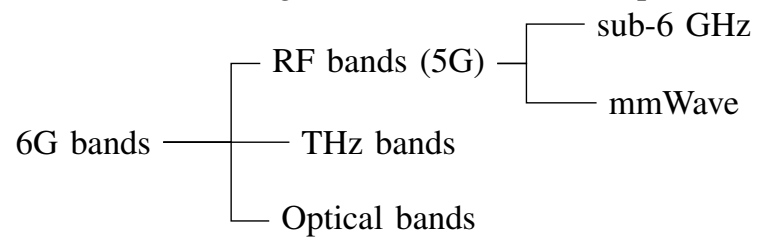

Channel propagation characteristics, use cases, and required hardware for these bands are very different.

- IoT dominant: By 2030, cellular networks will be IoT dominant. Ericsson projects that there will be 5.9 billion cellular IoT connections in 2026, up from 1.7 billion in 2020 , and expressing a $23 \%$ compound annual growth rate [6]. All four types of IoT (massive, critical, industrial, and broadband) are growing exponentially. These devices are more and more going to connect to non-terrestrial, cellular, and wide area networks.

\section{Survey Scope and Related Works}

Beginning the digital transformation initiated by IoT, there are a few trends focus across academia and industry:

1) IoT creates true global connectivity. 


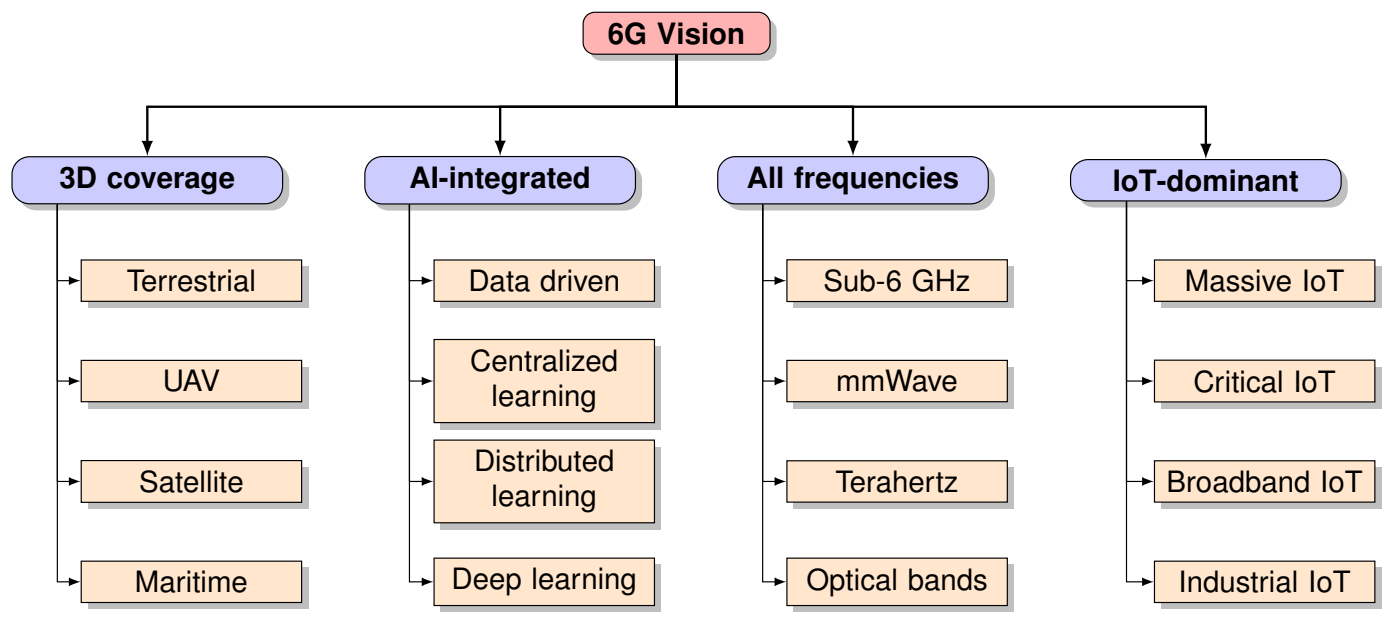

Fig. 5: A vision of new paradigm shifts in $6 \mathrm{G}$ communication networks.

2) $\mathrm{AI}$ is managing IoT devices and decision-making.

3) The 'edge' is becoming closer than ever before.

4) IoT security issues are rising continuously.

The high data rates, high reliability, global coverage, and low latency of $5 \mathrm{G}$ and beyond connections [25], coupled with cutting-edge AI technologies, such as deep learning and federated learning, will be indispensable toward making the reality of the above trend. On the other hand, there are several serious concerns in the areas of privacy and security. Further, the continual rise of IoT security issues besides global connectivity makes IoT security threats and privacy issues more alarming and necessitates novel solutions in this area.

1) Survey scope: This survey covers existing and emerging solutions related to all three big players, i.e., 5G and beyond access, integration of AI to IoT, and security issues. We review a wide range of technologies that enable IoT applications in cellular, wide-area, and non-terrestrial networks. Particularly we discuss the access networks over licensed and unlicensed spectrum. For cellular IoT, we cover the advances made to satisfy most of the 5G KPIs, including reliability, energy efficiency, massive connectivity (grant-free and nonorthogonal access), and latency. We also investigate the main IoT security/privacy threats and solutions. In addition, we elaborate on several emerging technologies and solutions that are expected to be a part of IoT in the era of 6G. Specifically, we overview various deep learning architectures and federated learning and explore the IoT applications that can benefit from these algorithms. Further, we discuss the remaining challenges and open issues that need to be addressed in the future. This survey does not cover traditional machine learning algorithms for IoT. It also does not go into the details of the IoT standards and infrastructure.

2) Related works: To the best of our knowledge, there is not a paper surveying IoT advances related to all 5G KPIs in the literature. There is also no paper about IoT solutions and architectures beyond $5 \mathrm{G}$ and their relation to those in $5 \mathrm{G}$. There are, however, a number of IoT surveys and tutorials related to certain topics covered in this survey. These surveys and their primary focus are listed in Table II. Our work is much more comprehensive and covers almost all 5G KPIs, as well as emerging AI and security solutions.

\section{E. Contributions}

Figure 6 depicts the structure of the paper. The contributions of this paper can be summarized as follows:

In Section II, a comprehensive overview of the KPIs and service requirements for IoT is presented. The various envisioned IoT use cases have a diverse set of service requirements, often in deep contrast with one another. Namely, we address the importance of latency and reliability for mission-critical IoT, survival time and service availability for cyber-physical and industrial systems, age of information (AoI) for sensor networks, battery lifetime and energy efficiency, connection density of IoT devices in the envisioned deployments, and finally, the required data rates and spectral efficiency. We address the outlined requirements for $5 \mathrm{G}$ as well as the visions for cyberphysical and IIoT applications while keeping an open eye on the visions for future generations of cellular and wide-area IoT technologies, particularly with respect to $6 \mathrm{G}$ developments.

In Section III, major IoT connectivity providers have been introduced and compared. Namely, we have categorized the existing technologies into solutions over the licensed and unlicensed spectrum. Then, for each category, major technologies in the market have been introduced. Finally, a comparative study of IoT technologies, including (i) communications characteristics, e.g. type of radio access and frequency; (ii) performance indicators, e.g. link budget and sensitivity; and (iii) major limitations, e.g. data rate and number of packets per day, has been presented. We further address the significant but challenging potentials of mmWave access technologies for future high-rate IoT access. Moreover, we discuss relaying, especially in form of device-to-device (D2D) relaying, as an enabling scheme for extending coverage, improving spectral efficiency, and enhancing reliability for IoT systems. The role of cooperation between networks and devices is discussed as a necessary leap to enable efficient, low-energy, and yet 
TABLE II: Existing IoT surveys and tutorials and their primary focus

\begin{tabular}{|c|c|c|c|c|c|c|c|c|c|c|c|c|c|c|}
\hline Year & Reference & Main Topic & 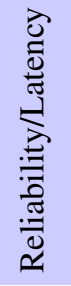 & 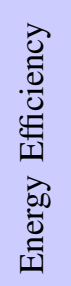 & 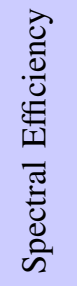 & 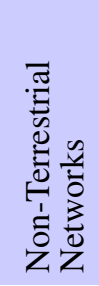 & 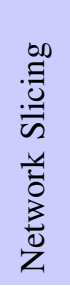 & 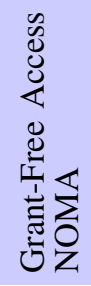 & 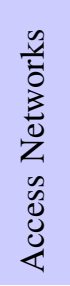 & 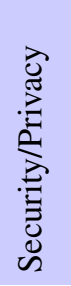 & 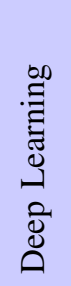 & 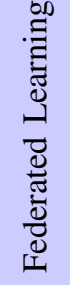 & 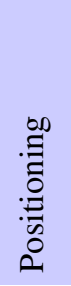 & 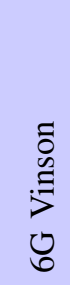 \\
\hline 2021 & This work & Cellular, wide-area, and 3D IoT & $\checkmark$ & $\checkmark$ & & $\checkmark$ & & $\checkmark$ & $\checkmark$ & $\checkmark$ & $\checkmark$ & $\checkmark$ & & $\checkmark$ \\
\hline 2021 & [26] & Network slicing for IoT & & & & & $\checkmark$ & & & & & & & \\
\hline 2021 & [27] & Positioning for IoT & & & & & & & & & & & $\checkmark$ & \\
\hline 2021 & [28] & Deep learning in IIoT & & & & & & & & & $\checkmark$ & & & \\
\hline 2021 & [29] & $6 \mathrm{G}$ vision for IoT & & & & & & & & & & $\checkmark$ & & $\checkmark$ \\
\hline 2020 & [30] & IoT forensics & & & & & & & & $\checkmark$ & & & & \\
\hline 2020 & [31] & NOMA-assisted MTC & & & & & & $\checkmark$ & & & & & & \\
\hline 2020 & [32] & Grant-free NOMA for IoT & & & & & & $\checkmark$ & & & & & & \\
\hline 2020 & [33] & DRL for autonomous IoT & & & & & & & & & $\checkmark$ & & & \\
\hline 2020 & [34] & Power-domain NOMA & & & & & & $\checkmark$ & & & & & & \\
\hline 2020 & [35] & ML in IoT security & & & & & & & & $\checkmark$ & $\checkmark$ & & & \\
\hline 2020 & [12] & Narrowband IoT PHY/MAC & & & & & & & $\checkmark$ & & & & & \\
\hline 2020 & [36] & Access control in IoT & & & & & & & & $\checkmark$ & & & & \\
\hline 2020 & [37] & Enabling technologies for IoT & & $\checkmark$ & & & & & $\checkmark$ & & & & & \\
\hline 2019 & [38] & $5 \mathrm{G}$ waveform for IoT & & & & & & & $\checkmark$ & & & & & \\
\hline 2019 & [39] & Enabling technologies IoT-5G & & & & & & & $\checkmark$ & & & & & \\
\hline 2019 & [40] & Energy-efficient IoT & & $\checkmark$ & & & & & & & & & & \\
\hline 2018 & [41] & IoT-enabled cyberattacks & & & & & & & & $\checkmark$ & & & & \\
\hline 2018 & {$[5]$} & Deep learning for IoT & & & & & & & & & $\checkmark$ & & & \\
\hline 2017 & [42] & Communication technologies & & & & & & & $\checkmark$ & & & & & \\
\hline 2017 & [43] & Narrowband IoT & & & & & & & $\checkmark$ & & & & & \\
\hline 2017 & [44] & IoT security & & & & & & & & $\checkmark$ & & & & \\
\hline 2016 & [45] & Aerial IoT & & & & $\checkmark$ & & & & & & & & \\
\hline 2015 & [46] & Enabling technologies & $\checkmark$ & & & & & & $\checkmark$ & $\checkmark$ & & & & \\
\hline
\end{tabular}

dependable communication service. The vision is to move away from rigid network architectures where all devices communicate directly with a network access point and enable a fluid architecture where devices and the network can form mesh-type sub-networks in an on-demand manner.

Section IV provides an overview of channel coding techniques for $5 \mathrm{G}$ and future wireless communication technologies for IoT. We first review the well-known channel coding techniques that have been considered for cellular systems and highlight their pros and cons for IoT applications. We then explain new requirements for channel coding and decoding mainly imposed by new IoT scenarios. This includes lowcapacity communications and channel state information (CSI)free communications. We then review some rate-adaptive coding techniques which can be effectively applied in these scenarios. We finally highlight the main requirements of the decoding techniques for resource-constraint devices.

Section $\mathrm{V}$ discusses massive connectivity solutions in $5 \mathrm{G}$ and beyond where solutions based on multiple access, random access, and new frequencies are considered. In multiple access, we discuss how new, non-orthogonal multiple access (NOMA) schemes in the uplink and downlink can increase the number of connected devices. We describe the state-of-the-art in power-domain NOMA as well as various code-domain NOMA schemes. Random access could be grant-based, grant-free, or unsourced. We argue that the last two are more suitable for massive IoT whereas the first one could be used for broadband IoT applications. Further, we describe how new frequencies (e.g., mmWave and $\mathrm{THz}$ ) are useful for broadband and critical IoT and can be used to accommodate such applications.

Section VI provides an overview of the most common security threats in cellular, wide-area, and non-terrestrial IoT deployments. We categorize possible attacks in terms of their attack medium: physical, software, or network attacks, and then discuss how emerging networking and database technologies can help address some of the security threats in IoT. Finally, going forward, we stress the need for better device authentication since traditional certificates/key exchange algorithms do no scale well. Better hardware-based authentication and key generation schemes, that can not be tampered with, are available in the FPGA/hardware trust research community and can be directly applied to future cellular, wide-area, and non-terrestrial networking devices.

Section VII covers deep learning, distributed learning, and federated learning for IoT. We being with a tutorial on various supervised and unsupervised deep learning models, their 


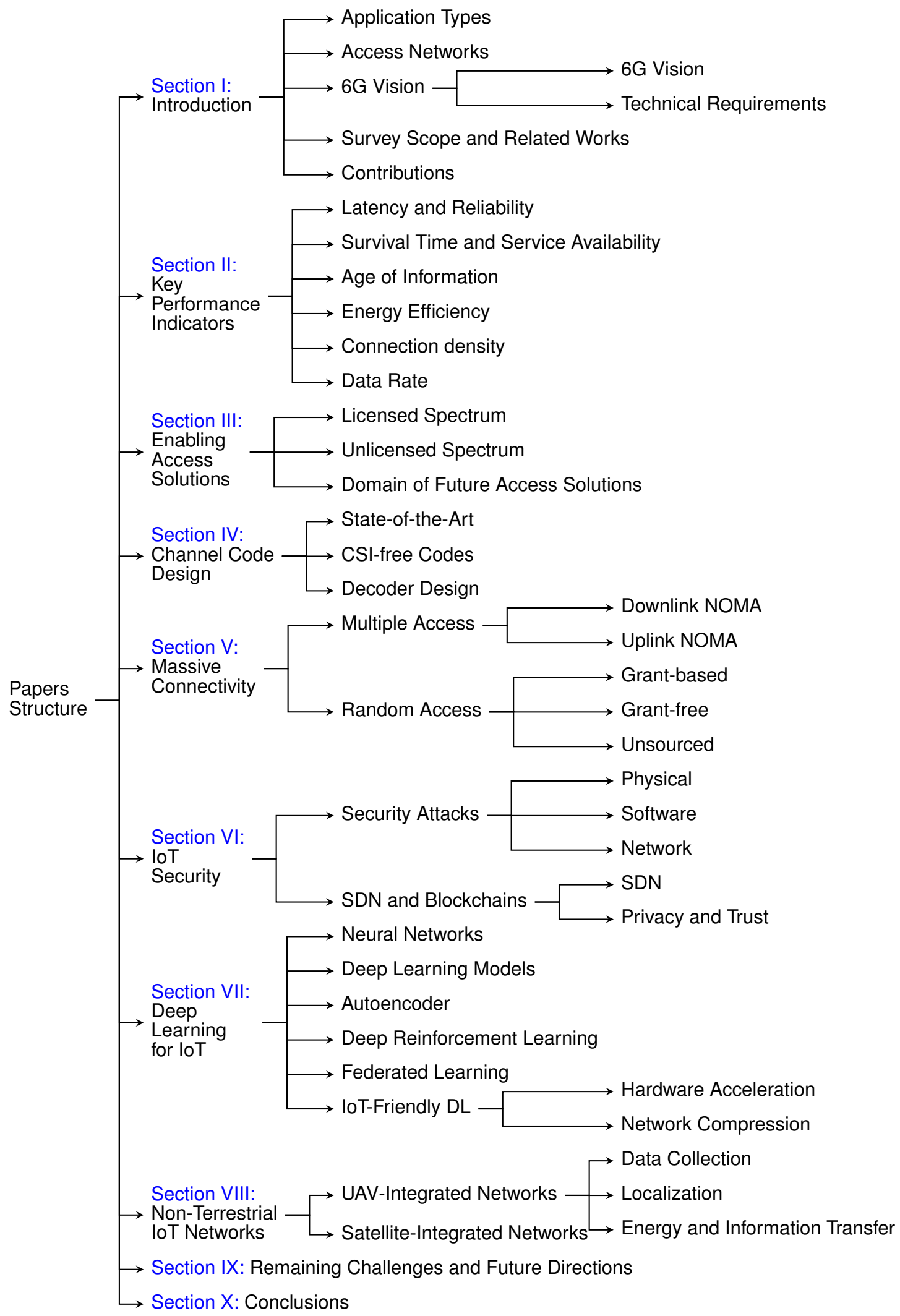

Fig. 6: Structure of the paper. 
advantages, and limitations for IoT uses. We then overview recent advances made using the above learning methods in various aspects of the IoT, including physical layer, network layer, and application layer. We also discuss characteristics and needs of the IoT-friendly deep learning. Particularly, we describe recent literature on network compression and hardware acceleration.

Section VIII provides an overview of the role of NTNs in IoT, the corresponding challenges, and opportunities. Section IX sheds light on the major remaining challenges of IoT communications, including the battery lifetime of IoT devices. Furthermore, several promising solutions for enabling further energy efficiency in communications have been explored. Among these solutions, great emphasis has been put on grantfree access for uplink-oriented communication and wake-up receiver for downlink-oriented communication, as two unexplored areas in cellular-based IoT communications, with great potential in saving energy for IoT devices.

A complete list of abbreviations is given in the Appendix.

\section{KEY PERFORMANCE INDICATORS}

The envisioned requirements for IoT are as diverse as the plethora of use cases that are associated with it. In this section, we provide a comprehensive overview of the requirements and KPIs that are outlined by technical expertise and industry visions. To this end, we build upon the outlines proposed in [47] as part of the $5 \mathrm{G}$ requirements, in [48] and [49] as the visions for cyber-physical control applications, and, in [18] as the visions for future generations of cellular telecommunications technologies.

\section{A. Latency and Reliability}

The initial momentum for low-latency wireless communication in the $5 \mathrm{G}$ era targeted mission-critical communication. The $5 \mathrm{G}$ vision aimed to achieve $1 \mathrm{~ms}$ latency for small size packets (see 3GPP definition illustrated in Fig. 7), used in applications such as remote surgery and machine control. Such applications also required high reliability of packet delivery. Therefore, in 3GPP Release 15, the main target of air interface, physical layer (PHY) and media access control (MAC) layer advancements was to enable $99.999 \%$ reliability within a $1 \mathrm{~ms}$ latency for 32 bytes packet size [50]. This initiated a category of use cases, commonly addressed under the umbrella term URLLC. New access techniques including flexible transmission time interval (TTI) size and mini-slots [51], grant-free channel access and preemptive scheduling (also know as punctured scheduling) [52] were deployed for low latency, while multi-connectivity with multiple access points [53], enhanced hybrid automatic repeat request (HARQ) techniques [54], and carriers and packet duplication [55] were introduced for reliability. The applications for critical communications have since expanded significantly, currently including use cases from energy management and smart grid control, health care and telesurgery, entertainment and gaming, and mobility and traffic control. Some of the new applications require lower latency, higher reliability and in some cases, significantly high data rates. In Table III, a few of those use cases are depicted according to [56] based on performance requirements of professional low-latency audio and video transport services. The terminology in Table III are defined as follows:

a) Transfer interval: the time difference between two consecutive transfers of packets from the application to the wireless communications system.

b) End-to-end latency: the time that takes to transfer a given piece of information from a source to a destination, measured at the communication interface, from the moment it is transmitted by the source to the moment it is successfully received at the destination.

As illustrated in Fig. 7, end-to-end (E2E) latency can generally consist of several delay components, including but not limited to, the propagation delay over the air and over $\mathrm{x}$-haul links, as well as the processing and queuing delay in switches and processing units. Among those, processing delays and $\mathrm{x}$-haul propagation delay are critical in the experienced round-trip delay [57]. For applications that sit far away from the user, the physical distance between the two ends of the communication link is a strict limiting factor on how small the E2E latency can get. For instance, the telesurgery entry in Table IV, denoted by motion control and haptic feedback, an upper bound of $20 \mathrm{~ms}$ is envisioned for E2E latency due to the fact that the communicating points may be sitting kilometers away from each other. On the other hand, some applications require sub-milliseconds E2E latency as listed in Table IV and Table III. For those applications, it is important to bypass as many delay components as possible of the ones depicted in Fig. 7. For instance, for motion control applications the controller and the actuators are usually located in close proximity of each other, thus allowing the communication link to be established by the central unit of the gNodeB (gNB), without the need to go through the core network. In such situation, the processing and routing of the traffic are controlled by the gNB in the edge cloud, as opposed to the core cloud. With proper design of the air interface, and ensuring a smooth queuing process at the $\mathrm{gNB}$, this will enable $\mathrm{E} 2 \mathrm{E}$ latency of sub-milliseconds in future cellular technologies.

Incidentally, the use case requirements are widely different in terms of reliability and latency, ranging from $10^{-4}$ to $10^{-10}$ packet error rate (PER), only in the presented examples. Furthermore, the differences in traffic types call for various types of radio access techniques, including stationary point-topoint, mobile device-to-device, and multi-cast and broadcast access.

For future cellular, wide-area, and non-terrestrial technologies in the $6 \mathrm{G}$ era, lower radio latencies of the order of $100 \mu \mathrm{s}$ at Gbits/s data rates are required to enable extreme networking solutions $[18,58]$. This will for instance enable replacing traditional industrial wireline connectivity solutions such as Sercos or EtherCAT. The requirement on reliability for some applications, such as in Table III, is significantly more challenging as compared to 5G URLLC. For others, such as cyber-physical applications, reliability is based on the downtime of the control application which is represented by the number of consecutive packet errors (for requirements concerning those applications, see Sec. II-B). Fig. 8 compares the latency and reliability performance of different wireless 


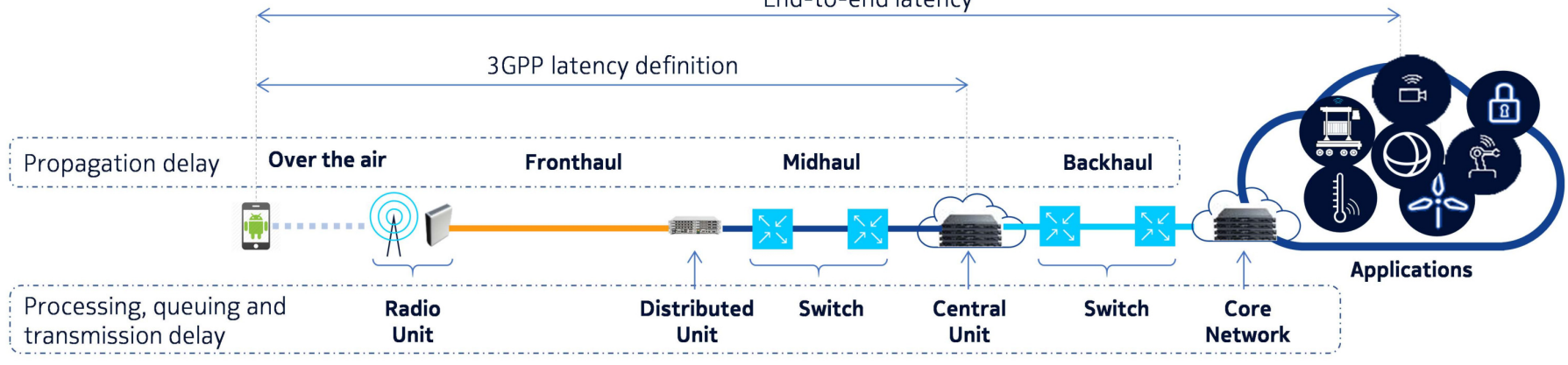

Fig. 7: Breakdown of the various elements of delay in E2E communication service latency.

TABLE III: Performance requirements of professional low-latency audio and video transport services [56]

\begin{tabular}{|l|l|l|l|l|l|l|l|}
\hline Packet error rate & $\begin{array}{l}\text { E2E } \\
\text { latency }\end{array}$ & $\begin{array}{c}\text { Transfer } \\
\text { interval }\end{array}$ & $\begin{array}{l}\text { Number } \\
\text { of UEs }\end{array}$ & $\begin{array}{l}\text { Uplink } \\
\text { data rate }\end{array}$ & $\begin{array}{l}\text { Downlink } \\
\text { data rate }\end{array}$ & Service area & Application \\
\hline \hline $10^{-6}$ & $750 \mu \mathrm{s}$ & $250 \mu \mathrm{s}$ & 30 & $5 \mathrm{Mbit} / \mathrm{s}$ & $\mathrm{NA}$ & $10 \mathrm{~m} \times 10 \mathrm{~m}$ & $\begin{array}{l}\text { Audio studio (periodic deterministic } \\
\text { traffic) }\end{array}$ \\
\hline $10^{-6}$ & $750 \mu \mathrm{s}$ & $250 \mu \mathrm{s}$ & 10 & $\mathrm{NA}$ & $3 \mathrm{Mbit} / \mathrm{s}$ & $30 \mathrm{~m} \times 30 \mathrm{~m}$ Audio and video production (peri- \\
odic deterministic traffic)
\end{tabular}

technologies addressed in this paper. The numbers in this figure must be taken as approximated based on the reported measurements and envisioned capabilities of different technologies in the literature. For the future generation wireless network, some extreme use cases are anticipated in Fig. 8 according to 6G URLLC [18], WiFi 7 promises to support low latency and ultra-reliable services [59], and the use cases in Table III and Table IV.

\section{B. Survival Time and Service Availability}

In the context of cyber-physical and industrial systems, dependable and real-time wireless communications are crucial to enable mobility. The wireless transformation in future industrial automation will reduce the bulk and cost of installation while enabling a highly flexible and dynamically re-configurable environment. This vision is shared by various other use cases too, including, smart grid, mobility and traffic, health care, entertainment, and gaming. The challenge is for the wireless communication system to be as dependable as a wired connection, i.e., at extremely high reliability while guaranteeing every time/everywhere service. Those qualities are realized in form of quality of service (QoS) characteristics introduced by $3 \mathrm{GPP}$ in the context of wireless cyberphysical systems, using the new notions of survival time and service availability $[49,60]$. Table IV shows a few example applications from [49]. The terminology in Table IV is defined according to the interaction between the application and the wireless communications system, as follows:

a) Survival time: the time that an application consuming a communication service may continue without an anticipated message. The anticipation is due to the periodic pattern of the traffic.

b) Communication service availability: the percentage value of the amount of time the E2E communication service QoS requirements-including E2E latency and survival time requirements-are satisfied for the application.

In light of these definitions, some rather interesting observations can be made from the examples provided in Table IV. The duration of the survival time depends on the application. In fact, for motion control systems responsible of controlling moving parts of machines, e.g., printing or packaging machines, the survival time is comparable to the transfer interval. This is also true for motion control with haptic feedback, e.g., in the case of robotic-aided telesurgery where the feedback provides tactile guidance for the patient body model. Mainly, the high-precision of operation that is vital in those use cases, translates into a tolerable duration of lost packets comparable to the transfer interval. On the other hand, in some applications with less stringent operation accuracy, such as remote control of a harbor crane, the survival time can be as large as six consecutive transfer intervals [61]. The immediate impact of introducing survival time as a QoS requirements of the wireless link is that burst of consecutive errors, longer than that of the survival time duration must be avoided. For instance, with survival time equivalent to one transfer interval, the system can tolerate one packet failure, however failure in delivering two consecutive packets results in the control application to fail.

Moreover, there is no direct relation between latency and survival time, where, depending on the use case and service 
area, those requirements may vary. For motion control applications within small areas, low E2E latency is achievable (physical distance allowing for it) and desirable as seen in the examples in Table IV. For example, the haptic-enabled controller node and the actuators of a motion control network can be located in the same room and connected to the same 5G base station, resulting in a short E2E link. As the distance between nodes increases (e.g., in case of a nationwide missioncritical network as depicted in Table IV), the E2E latency naturally increases too.

The introduction of survival time will result in a shift in design paradigm from typical link reliability-i.e., minimizing PER - to service availability-i.e., minimizing the chance of a burst of consecutive errors [62]. Recently, analysis of the consecutive packet failure rate and service availability for periodic traffic has gained attention in the literature. We refer to [63-66], where initial studies have been carried out on characterizing consecutive error rate statistics, both analytically and using empirical tools. In case of [64], the impact of several scheduling schemes on consecutive packet failure rate is empirically studied, suggesting a conservative linkadaptation for a link in survival mode.

A situation where a communication link has failed to deliver the last packet within the E2E latency budget is commonly referred to as survival mode. Motivated by the fact that cyberphysical applications can tolerate packet failures as long as the duration of consecutive failures is bounded by the survival time of the application, a proper strategy for survival mode operation is vital. A two-way communication link-which could consist of isochronous traffic between a controller and an actuator, or, a one-way traffic to the actuator followed by acknowledgment feedback - can inform the scheduler of packet failures [62]. Therefore, survival mode can trigger a more cautious transmission-i.e., with higher reliability-of the immediate next packet. This further motivates the network to discriminate between two modes of operation, namely the normal mode of operation which takes up most of the network operation time, providing the link with reasonable reliability levels, and the survival mode of operation, which expectantly occurs infrequently but requires a more reliable transmission, e.g., by means of increasing redundancy for the transmission. This analogy is deliberately reminiscent of the special treatment that an ambulance in a survival challenge receives on a congested highway, as opposed to normal cars.

\section{Age of Information}

A closely related QoS metric to survival time is the notion of AoI, which has been studied extensively in the literature, e.g., see [67-71], the survey in [72] and the references within. AoI characterizes the freshness of the information at the receiver end of a communication link by measuring the elapsed time since the freshest packet was generated at the transmitter end. It has been mostly studied for status update type of traffic, addressing the analysis of average AoI. This is particularly important in control applications, where the freshness of sensory information plays a key role in the decision-making of a central controller.
Compared to latency which is measured with respect to a fixed point in time when a data packet has been created, AoI is measured with respect to alteration of the state of, or the occurrence of an event in, the application or system that utilizes the communication service [73]. The notion of AoI can be coupled with survival time and service availability from Table IV by looking at the low percentiles of AoI distribution (one in millions and less, corresponding to the residue of time where service is not available) as follows: communication service is deemed available if the age of the freshest packet at the receiver is less than the sum of the E2E latency and survival time budgets.

\section{Battery Lifetime and Energy Efficiency}

Smart devices are usually battery-driven and a long battery life is crucial for them, especially for devices in remote areas, as there would be a huge amount of maintenance effort if their battery lives are short. For most reporting IoT applications, packet generation at each device is modeled as a Poisson process [74]. The energy consumption of a device can be then modeled as a semi-regenerative process where the regeneration point is located at the end of each successful data transmission epoch [75]. For a typical device, denote the stored energy in the battery at the reference time as $E_{0}$, static energy consumption per reporting period for data acquisition from environment and processing as $E_{\mathrm{st}}$, and energy consumption in data transmission as $E_{\mathrm{tx}}$. Then, the expected battery lifetime is derived as

$$
L(i)=\frac{E_{0} T_{i}}{E_{\mathrm{st}}+E_{\mathrm{tx}}} .
$$

Modeling of $E_{\mathrm{tx}}$ is closely coupled with the IoT technology, e.g. NB-IoT, and the multiple access scheme used for handling IoT communications. We can extend the definition and define the battery lifetime of an IoT application. The lifetime of an IoT application could be defined as the length of time between the reference time and when the service is considered to be nonfunctional. The instant at which the service becomes nonfunctional depends on the level of correlation between gathered data by neighboring devices. In critical applications with a sparse deployment of sensors, where losing even one node deteriorates the performance or coverage, the shortest individual battery lifetime may characterize the application lifetime. On the other hand, in denser deployments and/or when correlation amongst gathered data by neighboring nodes is high, the longest individual battery lifetime, or average individual battery lifetime could be used to characterize the application lifetime.

\section{E. Connection Density}

$5 \mathrm{G}$ is expected to support a connection of up to 1 million devices per square kilometer $\left(\mathrm{km}^{2}\right)$, ten times higher than that in $4 \mathrm{G}$. With the explosive growth in the number of IoT devices, the connection density of devices is expected to reach $10^{7}$ devices $/ \mathrm{km}^{2}$ in 2030 (see Fig. 5). Designing appropriate multiple access and random access techniques is the key to supporting massive IoT, also referred to as mMTC, in cellular networks. It should be highlighted that the characteristics of 
TABLE IV: QoS characteristics for cyber-physical and medical applications $[49,56]$.

\begin{tabular}{|c|c|c|c|c|c|c|c|}
\hline $\begin{array}{l}\text { Commun. } \\
\text { service } \\
\text { availability }\end{array}$ & $\begin{array}{l}\text { E2E } \\
\text { latency }\end{array}$ & $\begin{array}{l}\text { Message } \\
\text { size } \\
\text { [byte] }\end{array}$ & $\begin{array}{l}\text { Transfer } \\
\text { interval }\end{array}$ & $\begin{array}{l}\text { Survival } \\
\text { time }\end{array}$ & $\begin{array}{l}\text { Number } \\
\text { of devices }\end{array}$ & $\begin{array}{l}\text { Service } \\
\text { area }\end{array}$ & Application \\
\hline $\begin{array}{l}99.999 \\
99.99999\end{array}$ & $<\mathrm{TI}$ & 50 & $500 \mu \mathrm{s}$ & $500 \mu \mathrm{s}$ & $\leq 20$ & $\begin{array}{l}50 \mathrm{~m} \times 10 \\
\mathrm{~m} \times 10 \mathrm{~m}\end{array}$ & Motion control \\
\hline $\begin{array}{l}99.9999 \quad- \\
99.999999\end{array}$ & $<\mathrm{TI}$ & N/A & $\leq 1 \mathrm{~ms}$ & $3 \times \mathrm{TI}$ & $2-5$ & $\begin{array}{l}100 \mathrm{~m} \times 30 \\
\mathrm{~m} \times 10 \mathrm{~m}\end{array}$ & $\begin{array}{l}\text { Wired-2-wireless } 100 \text { Mbps link re- } \\
\text { placement }\end{array}$ \\
\hline 99.999999 & $<2 \mathrm{~ms}$ & $\begin{array}{ll}250 & \text { to } \\
2000 & \end{array}$ & $1 \mathrm{~ms}$ & $1 \mathrm{~ms}$ & 1 & room & Motion control and haptic feedback \\
\hline 99.9999 & $\begin{array}{l}< \\
\mathrm{ms}\end{array}$ & $\begin{array}{ll}250 & \text { to } \\
2000 & \end{array}$ & $1 \mathrm{~ms}$ & $1 \mathrm{~ms}$ & $\begin{array}{l}<\underset{1000 \mathrm{~km}^{2}}{2 \text { per }} \\
\end{array}$ & national & Motion control and haptic feedback \\
\hline 99.999999 & $<2 \mathrm{~ms}$ & 50 & $2 \mathrm{~ms}$ & $2 \mathrm{~ms}$ & $>2$ & $100 \mathrm{~m}^{2}$ & $\begin{array}{l}\text { Mobile Operation Panel: Haptic } \\
\text { feedback data stream }\end{array}$ \\
\hline$>99.99999$ & $<1 \mathrm{~ms}$ & $\begin{array}{l}1500 \text { to } \\
9000\end{array}$ & - & $8 \mathrm{~ms}$ & 1 & $100 \mathrm{~m}^{2}$ & $\begin{array}{l}\text { Imaging/video traffic for medical ap- } \\
\text { plications }\end{array}$ \\
\hline
\end{tabular}

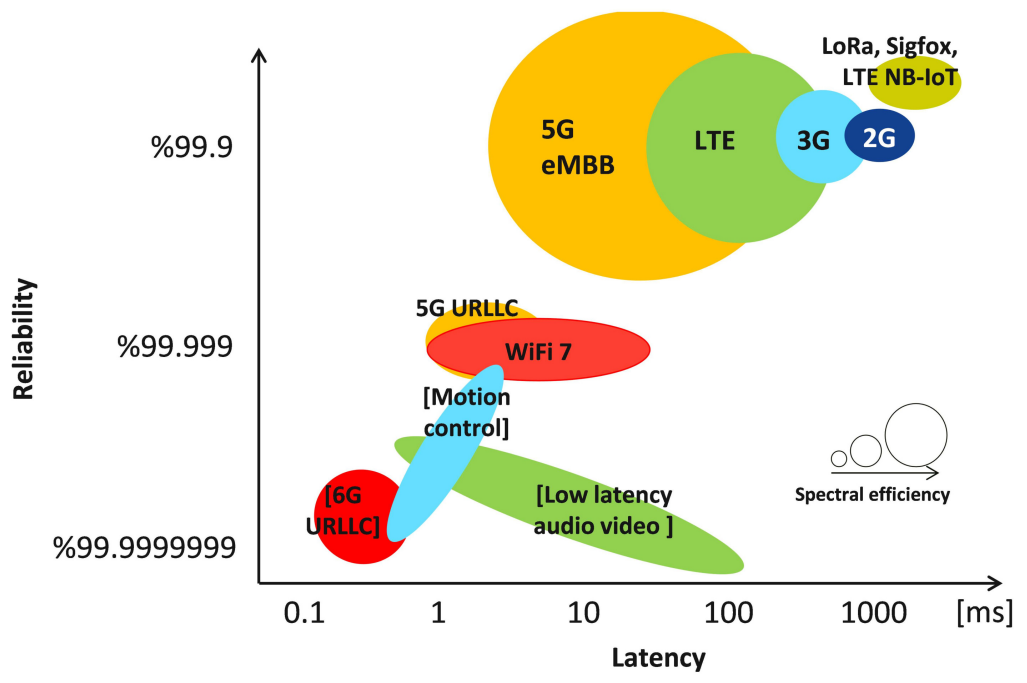

Fig. 8: Latency and reliability capability of different technologies. Envisioned use cases and technologies are noted in brackets.

massive access for cellular IoT are very different from those of eMBB and URLLC, the other two use cases of 5G. In particular, often, eMBB is for broadband IoT whereas URLLC is for critical IoT. In the former, providing high data rates is important whereas in the latter guaranteeing ultra-reliable low-latency communications is the main goal. Representative examples of the two use cases are augmented/virtual reality and autonomous driving, respectively. The main concern of mMTC is, however, to ensure wide coverage and low power consumption such that IoT sensors could communicate for a long period of time. More accurately, massive IoT is characterized by

- sporadic traffic

- small payload

- low power consumption

- limited capacity

- extensive coverage

With sporadic traffic and small payload, the traffic will be busty, and short-packet transmission will be preferred. Further, idle devices do not access the network in order to save power and extend the battery life. Then, low power standards, like LTE-M and NB-IoT, are necessary for battery lifetime. Limited processing and storage capacity of these devices implies that sophisticated signal processing cannot be implemented. With the above requirements of massive access, in beyond 5G cellular IoT, multiple access is evolving to massive access [76] for such devices.

\section{F. Data Rate and Spectral Efficiency}

A few KPIs are related to the data rate in one way or another. These are peak data rate, user experiences peak data rate, and spectral efficiency. 5G networks are expected to offer peak data rates of up to $20 \mathrm{Gbps}$ whereas peak data rates for $6 \mathrm{G}$ will be about 1,000 Gbps (see Table I and Fig. 4). Also, compared to $5 \mathrm{G}$, user experienced data rate is expected to increase about an order of magnitude in 2030. Further, spectral efficiency must improve two to three times. the technologies facilitating these escalations are mmWave, $\mathrm{THz}$, and massive multipleinput multiple-output (MIMO). This paper is not meant to discuss solutions related to the data rate in detail.

\section{ENABLING ACCESS SOlutions}

The continuing growth in demand for connecting smart things has encouraged different industries to investigate evolutionary and revolutionary connectivity solutions for serving 


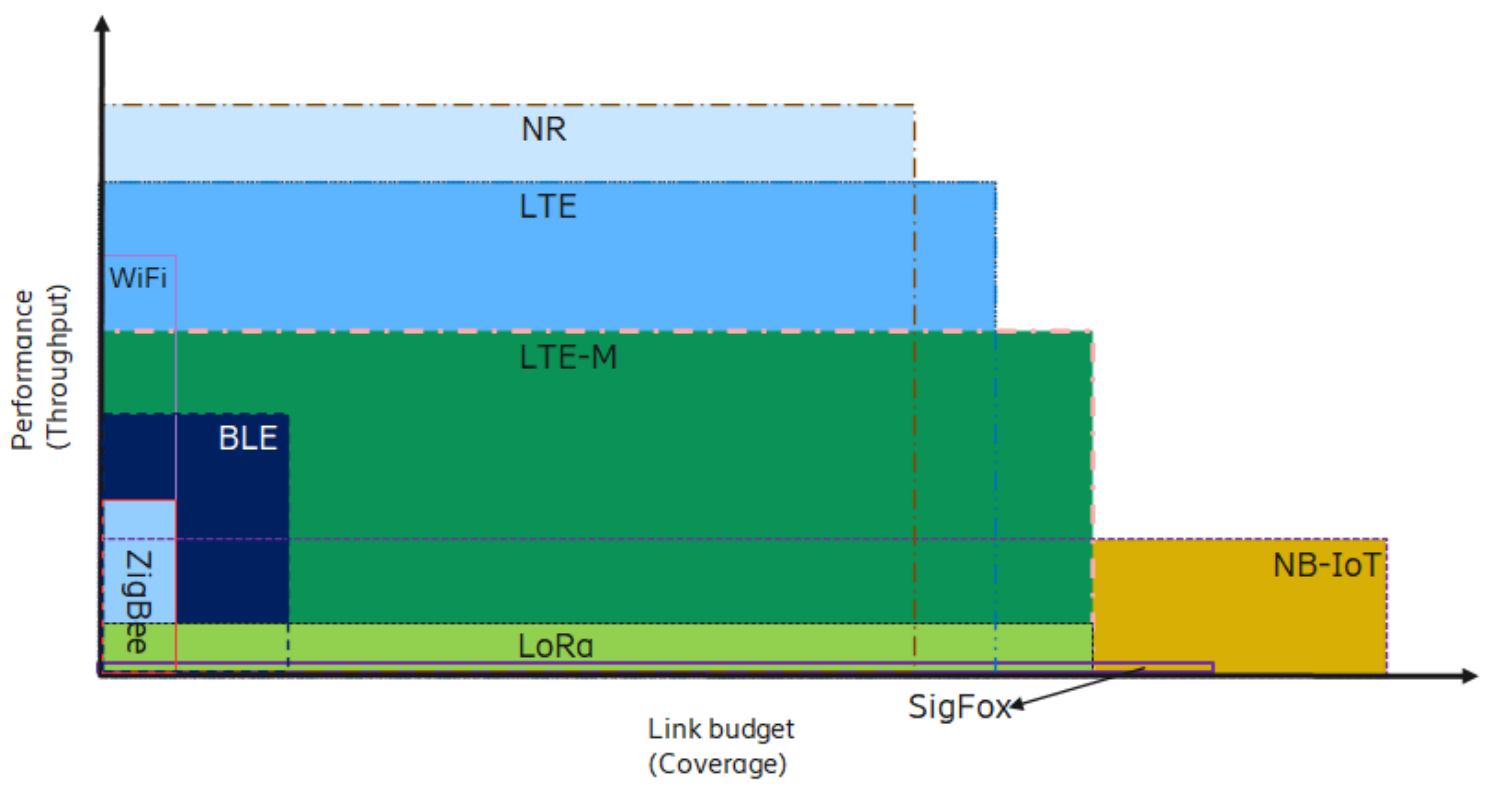

Fig. 9: A comparison of throughput and coverage of different connectivity technologies

IoT communications [77]. The major IoT connectivity solutions can be classified into two classes based on the criteria of data rate and transmission range requirements. Range-wise for instance, Zigbee like protocols are well-suited for mediumrange IoT applications and complement WiFi-like IoT use cases, home automation, smart lighting and energy management just to name few. Additionally, Bluetooth and Bluetooth low-energy (BLE), so called Bluetooth Smart are welloptimized for short-range consumer IoT applications owing to their low power dissipation, which are standardized in the Wireless Personal Area Networks, where BLE mainly targets for fitness and medical wearables, as well as for smart home devices. Another medium-range WiFi derivative for assisting IoT technologies is the WiFi-HaLow (IEEE 802.11ah), which is capable of providing reasonable coverage and less energy dissipation compared to WiFi. However, mainly due to security issues Wifi-HaLow has not taken the deserved attention of the industry. Finally, radio-frequency identification (RFID) can be exploited as part of the IoT technologies owing to its very small amount of data transmission requirement within a very short distance. RFID is mainly used for real-time monitoring of inventory and assets in order to provide efficient stock management and production planning, which is now taking its place for self-checkout, smart mirrors and shelves as emerging IoT applications. After having a brief discussion on shortrange IoT technologies without emphasizing on the data rate requirements, we focus our attention on the long range IoT wireless technologies, which can be further divided into two categories based on their usage of radio spectrum: i) solutions over the licensed spectrum like LTE category M and NBIoT [78]; and ii) solutions over the unlicensed spectrum like SigFox and LoRa wide area network (LoRaWAN) [79]. In the following, we investigate these categories in details.

\section{A. IoT Communications over Licensed Spectrum}

Cellular networks are expected to play a significant role in large-scale deployment of IoT solutions because of their ubiquitous coverage and roaming capability $[80,81]$. Following the early-stage predictions of a huge expected increase in the IoT traffic by Cisco [82], network providers have started devising solutions for adopting IoT traffic over their networks in order to find new revenue streams [83]. Generally speaking, the IoT traffic over cellular networks is served in three different ways:

- it is served along with the legacy traffic, i.e. it passes the same procedures as non-IoT traffic;

- it is served along with the legacy traffic but using new protocols aiming at enabling lo power/cost IoT connectivity;

- it is served over a dedicated pool of radio resources using IoT-specific algorithms and protocols.

The former, i.e. serving IoT and non-IoT traffic streams using the same resources and procedures, is usually the case in $2 \mathrm{G}$ networks, and it out of our scope here. The other two cases are investigated in the sequel.

1) IoT/non-IoT traffic serving over the same resources using dedicated communications protocols: A legacy connectivity procedure in LTE/LTE-Advanced (LTE-A)/LTE-Pro networks consists of different steps, including synchronization, connection establishment, authentication, scheduled data transmission, confirming successfulness of data transmission, and connection termination, as described in details in [84]. The random access channel (RACH) of LTE networks is the typical way for IoT devices to start connection with the base station (BS), and attach to the network for following data transmissions. Over the RACH, each device chooses a preamble randomly from a set of broadcasted preambles for access establishment with the BS. Since the total number of available preambles at each time is limited, the connection over $\mathrm{RACH}$ introduces collisions and energy wastage, especially 
when a multitude of of devices try simultaneously to get access to the network, e.g. after a power outage in a building or alarm [84]. Once a device successfully passes the RACH procedure and becomes connected, it can send scheduling requests to the BS over the physical uplink control channel (PUCCH). Now, the BS performs scheduling and responds to the device by sending the scheduling grantsover the physical downlink control channel (PDCCH). Then, a granted device can send its data over the physical uplink shared channel (PUSCH) resources to the $\mathrm{BS}$. In this procedure, even while there is no communications between the device and the BS, device needs to be ready for listening to the PDCCH for potential downlink data, which is heavily battery consuming. Then, one solution for energy saving is to let device get into sleeping mode for a certain period of time and wakes up again for checking if there is there is any data queued at the network side, and getting into the sleeping mode again after communications (or lack of queue data). This kind of periodic transfer between sleep and wake up modes is called discontinuous transmission (DRX). DRX provides energy saving at user device by introducing short and long sleep periods during. For sure, configuration of these sleep/wake up periods to the traffic pattern plays a crucial role in energy efficiency of IoT communications. However, the existing connection establishment, scheduling, scheduled data transmission, and DRX protocols in the legacy networks have been neither designed nor optimized for IoT communications. Hence, while they perform well for a limited number of longlasting streaming/call sessions, for constrained IoT devices with a massive number of short-length packets (communication sessions) these procedures could be the bottlenecks. Thus, congestion in serving IoT communications, including radio network congestion and signaling network congestion, is likely to occur [85]. Towards isolating the impact of IoT communications from the legacy communications, the use of dedicated resources for IoT communications has been proposed in LTE-Rel 13 onwards, to be discussed in the following.

2) IoT traffic serving over dedicated resources and communications protocols (LTE-M and NB-IoT): From LTE-Rel 12, the evolution of LTE for serving low-cost IoT communications over dedicated resources has been started, and it has been continued in Release 13 [86]. In Release 13, LTE$\mathrm{M}$ and NB-IoT systems have been introduced. These systems provide IoT connectivity over dedicated $1.4 \mathrm{MHz}$ and $200 \mathrm{KHz}$ bandwidths, respectively [86]. In the following, we focus on the NB-IoT, as the leading IoT connectivity solution from the 3GPP for low-cost low-power IoT devices.

NB-IoT represents a big step towards accommodation of low-cost low-power IoT devices over cellular networks [87]. Communications in NB-IoT takes place in a very narrow bandwidth, i.e. $200 \mathrm{KHz}$. This results which results in more than $20 \mathrm{~dB}$ extra link budget compared to the standard LTEA systems [87]. Thanks to this link budget, NB-IoT enables smart devices deployed in remote areas, e.g. rural areas, to directly communicate with the base stations, as compared to the gateway/relay-based solutions. In the above, we discussed that the legacy communication protocols and logical channels have been designed and optimized for data/bandwidth-hungry applications, e.g. $20 \mathrm{MHz}$ in LTE-A. Then, NB-IoT introduces five novel narrowband physical channels, including: narrowband physical random access channel (NPRACH); narrowband physical uplink shared channel (NPUSCH); narrowband physical downlink shared channel (NPDSCH); narrowband physical downlink control channel (NPDCCH); and narrowband physical broadcast channel (NPBCH) [88, 89]. Moreover, NBIoT introduces four new physical signals: narrowband reference signal (NRS); narrowband primary synchronization signal (NPSS), demodulation reference signal (DMRS), which is sent along with user data on NPUSCH; and narrowband secondary synchronization signal (NSSS). In NB-IoT, a detached device which aims at connecting to the network, follows the folloing procedures. It first listens for receiving cell information, e.g. NPSS and NSSS, through which, it makes itself synchronized with the NB-IoT BS. Then, it waits for the NPRACH to establish connection over random access resources, for NPDCCH to receive the scheduling response, and finally for NPUSCH/NPDSCH to send/receive data to/from the BS.

In contrast to the LTE networks, in NB-IoT IoT devices are allowed to go from the idle mode to the deep sleep mode during which, device is still registered with the BS. Then, upon waking up, device becomes connected and ready for communications by sending/receiving a fewer number of handshaking messages [78]. This new functionality enables saving a considerable amount of energy, especially in the connection establishment procedure and resource reservation [90]. Moreover, coverage extension has been devised for NBIoT systems, which is achieved by time-domain repetition of signals. Towards this end, BS assigns each device to a coverage class, from a limited number of defined classes, based on several parameters, including the average experienced deviceBS path-loss. This is the coverage class that characterizes the number of replicas per data packet in communications. For example, based on the specifications in [89], each device belonging to group $i$ shall repeat $2^{i-1}$ times the preamble message transmitted over the NPRACH, where $i \in\{1, \cdots, 8\}$. This repetition, enables NB-IoT systems to offer deep coverage to most indoor areas [78].

In LTE Rel.13, 3GPP standardization body introduced extended DRX (eDRX) and power-saving mode (PSM), as two main components for NB-IoT and LTE-M [91-93]. eDRX enables IoT application developers to dynamically change how long an IoT device should spend in the sleep period. The minimum sleep times for eDRX are 320 milliseconds (ms) for LTE-M and 10.24 seconds for NB-IoT; and the maximum sleep times are 43 minutes LTE-M, and 3 hours for NB-IoT $[91,94]$. Considering the fact that in the legacy DRX, the sleep times are typically 1.28 seconds or 2.56 seconds, one observes how eDRX can significantly help IoT devices to save energy. Moreover, while in the DRX scheme, DRX parameters are tuned by the network, the eDRX approach enables application developers to set and change the eDRX configurations, which brings much more flexibility to the applications. Finally, in eDRX, device can listen for pending data at the network side without the need to establish a full network connection, which also saves significant energy for the device [94]. In contrast to eDRX, in PSM device is goes to a very-low power 
consumption mode in which, there is only an active timer for counting the length of the PSM state [91]. The length of PSM could be as high as 413 days, which brings significant saving for IoT devices that rarely need data transmissions. In the PSM mode, once the device wakes up and sends data, it is reachable over 4 consecutive time windows. In these time windows, network can ask the device to stay awake for a longer period of time, or send data to the device [91,94].

In order to further improve NB-IoT systems, in [95, 96], preamble design for improving RACH of NB-IoT has been investigated. Energy efficient radio resource scheduling of IoT communications has been investigated in [97]. For NB-IoT deployment in LTE guard-band, a deployment option which introduces external interference to the IoT communications, coverage extension has been investigated in [98]. In [90], energy consumption in data transmission in different coverage regimes, including normal, robust, and extreme cases, has been studied. Then, in robust and extreme regimes, the extra coverage by signal repetition in the time domain has been characterized. When compared to the legacy LTE systems, energy consumption analysis in [90] illustrates that NB-IoT systems can significantly reduce the consumed energy in connection establishment due to their capability in easier move between sleep and active modes. Regarding the fact that we are still waiting for large-scale deployment of NB-IoT networks and wide access of IoT devices to its services, there is a lack of a comprehensive study on capacity, energy efficiency, and scalability of NB-IoT systems. Especially, when it comes to coexistence of coverage classes with different repetition orders over the same system, the work in [99] has shown that NB-IoT enhances energy efficiency for far-away devices at the cost of increasing latency for nearby devices, which calls for further investigation.

\section{B. IoT Networks over Unlicensed Spectrum}

From first-generation (cellular networks) (1G) to $4 \mathrm{G}$, the telecommunications industry has spent a great deal of resources investigating how to realize high-throughput infrastructure for serving data-hungry applications, and IoT solutions have been out of main scope. Along with telecoms' transition from $2 \mathrm{G} / 3 \mathrm{G}$ to $4 \mathrm{G}$ networks (2010-2020), some 3GPP-independent companies have started entering the lowpoer wide-area market, by providing large-scale IoT connectivity solutions, especially over the unlicensed spectrum, e.g. SigFox and LoRaWAN. While reliability of communications over the unlicensed spectrum is a big challenge due to grantfree access to radio resources, the simplified connectivity in these solutions has significantly reduced the cost per device. It has also capable of increasing battery lifetime of devices. Then, they are able to deliver their service at low cost for IoT applications in which, reliability of communications could be traded. Regarding the fact that energy consumption in synchronization, connection establishment, and signaling (in the cellular networks) is comparable with, or even higher than, the energy consumption in the actual data transmission (which is the only source of energy consumption in solutions leveraging grant-free access over unlicensed spectrum) [90,
100], IoT connectivity over cellular networks is also expected to move towards more relaxed radio access schemes, as investigated in [101]. The low-power wide-area (LPWA) IoT solutions over the unlicensed spectrum, along with cellular NB-IoT and LTE-M solutions, are expected to share 60 percent of the IoT market among themselves, a number that is expected to grow over time, and is expected to bring new revenue streams to network providers. Hence, the competition between IoT connectivity technologies (with low to medium reliability and datarate requirements ${ }^{4}$ ) is becoming intense [79]. In the sequel, SigFox and LoRaWAN are introduced as two dominant LPWA IoT solutions over the unlicensed spectrum. In these solutions, data is collected by local access points (APs), which the point of access to the Internet for IoT devices. These APs are connected to the Internet infrastructure through wired or wireless backhauling. The IoT end-user accesses the gathered data by devices through the IoT server, and potentially sends commands back to them, again through the gateways. Both LoRaWAN and SigFox use the industrial safety medical (ISM) spectrum band for communications (detailed description of frequency bands could be found in the following subsections as well as Table V). Over the ISM bands, any device can communicate as long as it complies with the fair spectrum use regulations [79]. The regulations vary from one sub-band to another, from one region to another, and are mainly restricting the effective radiated power, number of transmissions per device per day, as well as the spectrum access method [102]. Based on [102], the spectrum access can be either listenbefore-talk, or the duty cycle. In the former, devices start transmissions in case the sensed channel is free, while in the latter, devices start transmission once it has data to transmit by considering the constraint that it cannot transmit in more than $x \%$ of the time, where $x$ is determined from the regulations. The communications protocols used in the lower layers of SigFox and LoRaWAN are discussed in details in the sequel.

1) SigFox: Founded in 2009 in France, SigFox is the first widely announced proprietary IoT technology. The SigFox technology aims at providing low-cost low-power IoT connectivity over the ISM band. Thanks to its ultra-narrowband signals, as low as $100 \mathrm{~Hz}$, SigFox is able to cover large outdoor areas, and even is able to provide high link budget for indoor coverage, as shown by experimental results in [103]. Once a SigFox device has a packet to be transmitted, one copy of the packet is transmitted immediately, and two other copies of the packet are sent consequently with a semi-random frequency hopping over a $600 \mathrm{KHz}$ system bandwidth $(6000$ times more than the signal bandwidth) [102,104]. In the first releases, SigFox was only supporting uplink communications; however, from 2015 it also supports uplink-initiated downlink communications. In other words, once a device transmits data and requests for a response from the application, the AP sends the acknowledgment (ACK) as well as the queued data waiting for the device from the server side [100]. As per other grant-free radio access solutions, SigFox doesn't require pairing for starting communications. This on the other hand

\footnotetext{
${ }^{4}$ Later in Table V, we will see that cellular solutions have no competitor in medium to high reliability and datarate performance regimes.
} 
means that there is no need for message exchanges (and energy consumption) in synchronization, authentication, and connection establishment $[102,104]$. The duty cycle for a SigFox AP is $10 \%$, while the duty cycle for devices is $1 \%$. This means that a SigFox AP can transmit data at most in $10 \%$ of the time, as compared to a devices which can do it in $1 \%$ of the time. The difference in duty cycling comes from the fact that AP and devices are working on different sub-bands of the ISM band. Hence, they comply with different regulations. From this duty cycling puts an upper-limit of 140 messages of 12-bytes per device per day, which makes SigFox unfavorable for applications with more data exchange requirements (also for applications with immediate Ack messages per packet, or with delay-bounded availability constraints). Monitoring of the status of the network and manage operations of the APs is managed at the core of SigFox networks. SigFox modules are available at low cost, e.g. $2 \$$ per module [105], to be used in various IoT devices. The cost comparison in [105] shows that the cost of a SigFox module is almost one-third of a long range (LoRa) module (which is more complex than a SigFox module, as we will see in the sequel), and one-fifth of a cellular IoT module (which is expensive due to many complex functions embedded to comply with cellular standards).

2) LoRaWAN: LoRaWAN leverages LoRa communications in its lower layers of protocol stack. LoRa is utilizing a propitiatory chirp spread spectrum (CSS) as a modulation to mitigate the potentially severe interference on the unlicensed bandwidth and has been claimed to be robust against multipath fading and Doppler shifts to some extent [106]. In contradictory with the SigFox technology, the LoRa gateway is open source, i.e. may build her own LoRaWAN gateway (e.g. on top of micro-controllers like Arduino), connect it to the Internet, and use it for long-range data gathering from LoRa-compatible IoT devices (or even built her own devices again on top of micro-controllers).

The LoRaWAN servers are managing the allocation of spreading factors (SFs) to connected devices in a centralized way, in order to balance the cell-wide data-rate and reliability of communications. SFs, denote the number of chirps used to encode a bit, and are ranged from 7 to 12. In LoRa, the higher the chirp rate is, the better reconstruction of the received signal is achieved, while on the other hand, an increase in the SF stretches the transmission time [107], and hence, increases the probability of collision with another data transmission (increased transmission time). Regarding the ever-increasing rate of deployment of IoT solutions over the ISM band, and the uncontrolled access to radio resources over this spectrum, a high level of resilience to sporadic interference has been introduced in LoRa. In fact, the main feature of CSS, which is used in the LoRa modulation as a simplified version of a satellite communication protocol, is that signals with different SFs can be almost distinguished and received simultaneously, even if they are transmitted at the same time and on the same frequency channel [108]. LoRaWAN 3 classes in which, communications parameters and capabilities are different. Especially in class A, downlink transmission is possible after data transmission of a device during two non-overlapping and consecutive dedicated time intervals, which is called receive windows in the context of LoRaWAN [109].

3) Comparison of different technologies: Table V represents a comprehensive comparison of enabling solutions for wide-area IoT communications, i.e. SigFox and LoRaWAN (over the unlicensed spectrum), and LTE-M and NB-IoT (over the licensed spectrum). A graphical illustration of the throughput/coverage comparison has been depicted in Fig. 9. In this figure, one further observes which areas of range and data-rate (as two main performance indicators) have been covered by different technologies.

\section{Domain of Future Access Solutions}

1) New Spectra: Allocating new frequencies is a straightforward solution for increasing the number of connections in the network. A good example is the introduction of mmWave bands (30-300 GHz) in 5G networks. Similarly, $\mathrm{THz}$ band (0.1-10 THz) and optical spectrum are expected to be exploited in beyond $5 \mathrm{G}$ for ultra-high data rates [22-24]. However, due to their wide bandwidth and high penetration loss, mmWave frequency bands are generally not considered for massive connectivity, but they can be used for broadband IoT and critical IoT.

a) mmWave IoT: High ranges of radio frequency, well above the single-digit gigahertz frequencies used for previous generations, are subject of technology development and deployment in the time of $5 \mathrm{G}$ mobile networks and beyond. Currently, the mmWave bands includes several gigahertz of licensed and unlicensed bandwidth in the 24-43 GHz range, as well as above $57 \mathrm{GHz}$, which has been made available by different regulatory bodies across the world [112].

Propagation characteristics for mmWave bands have been studied extensively for urban and indoor scenarios [113-116]. The main challenge that is inherently coupled with mmWave is higher atmospheric absorption, causing large propagation loss. Moreover, mmWave faces sever blocking due to limited amount of diffraction around objects. Thanks to small wavelength, tens of antenna elements can fit on a single small-sized array and enable high gain beamforming. Nevertheless, the high propagation loss limits the communication range to several tens of meters up to a few hundred meters, which makes mmWave well suited for indoor networks. Consequently, ubiquitous coverage in mmWave requires a sufficiently high density of radio APs and enabling multi-link communications such that the user can simultaneously communicate with multiple radio APs, also known as transmission reception points (TRPs) [117]. Such densification of access points can improve the chances for a user to experience a non-blocked link to at least one TRP at a time, especially in environments with dens and moving clutter of blocking objects.

The wide spectrum bandwidths available in mmWave has made it mostly known as a solution for significantly high data rates of multigigabits [113]. Recently, other aspects of mmWave, such as the large frequency diversity and beamforming gain potentials, have raised the attention of researchers towards enabling mmWave URLLC in IIoT [118, 119]. Specifically, the large mmWave bandwidths and contained delay spread offers the attractive possibility to shrink the TTI to 
TABLE V: A comparison of different wide-area IoT enablers

\begin{tabular}{|c|c|c|c|c|}
\hline Feature & SigFox & LoRaWAN & LTE-M & NB-IoT \\
\hline Frequency $(\mathrm{MHz})$ & ISM (865-924) & ISM $(433 / 868 / 915)$ & Licensed $(410, \cdots, 5900)$ & $\begin{array}{l}\text { Licensed }(410, \cdots, 5900) ; \\
\text { also out of LTE-band }\end{array}$ \\
\hline $\begin{array}{l}\text { Signal bandwidth } \\
\text { (BW) }\end{array}$ & $100 \mathrm{~Hz}$ & $125-500 \mathrm{KHz}$ & $\begin{array}{l}1-20 \mathrm{MHz} \text { (downlink); } \\
1.5,5 \mathrm{MHz} \text { (uplink) }\end{array}$ & $\begin{array}{l}180 \text { (LTE band); } 200 \text { (GSM } \\
\text { band) KHz }\end{array}$ \\
\hline $\begin{array}{l}\text { uplink/downlink } \\
\text { support }\end{array}$ & yes/limited & yes/yes & yes/yes & yes/yes \\
\hline Packet size & $\begin{array}{l}8 \text { (downlink); } 12 \text { (up- } \\
\text { link) bytes }\end{array}$ & 19-250 bytes & 2984-6968 bits & 16-2536 bits \\
\hline Modulation & GFSK & LoRa (proprietary) & B/QPSK;16-64QAM & $\begin{array}{l}\text { QPSK (uplink/downlink); } \\
\text { BPSK (uplink) }\end{array}$ \\
\hline Radio access & $\begin{array}{l}\text { Random } \\
\text { FDMA/TDMA }\end{array}$ & $\begin{array}{l}\text { Random } \\
\text { FDMA/TDMA }\end{array}$ & $\begin{array}{l}\text { FDMA (downlink); } \\
\text { single-carrier FDMA } \\
\text { (SC-FDMA) (uplink) }\end{array}$ & $\begin{array}{l}\text { OFDMA (downlink); SC- } \\
\text { FDMA (uplink) }\end{array}$ \\
\hline BW constraints & $\begin{array}{l}\text { Fair use of ISM: } 14 \\
\text { packets/day }\end{array}$ & $\begin{array}{l}\text { Fair use of ISM: } 30 \\
\text { sec/day (uplink); } 10 \\
\text { packets/day (down- } \\
\text { link) }\end{array}$ & Pay as you go & Pay as you go \\
\hline Tx power $(\mathrm{dBm})$ & $\begin{array}{l}\text { 14, } 22 \text { (uplink); 27, } 30 \\
\text { (downlink) }\end{array}$ & $\begin{array}{ll}14-30 & \text { (region- } \\
\text { dependent) }\end{array}$ & 20 & $14,20-23$ \\
\hline $\begin{array}{l}\text { Downlink sensitiv- } \\
\text { ity }\end{array}$ & $-130 \mathrm{dBm}$ & $-137 \mathrm{dBm}$ & $-132 \mathrm{dBm}$ & $-141 \mathrm{dBm}$ \\
\hline Link budget $(\mathrm{dB})$ & 163 & $\begin{array}{l}154 \text { (uplink); 154- } \\
157 \text { (downlink, } \\
\text { region-based) }\end{array}$ & 155 & $\begin{array}{l}164 \text { (20-23 dBm Tx power); } \\
155 \text { (14 dBm Tx power) }\end{array}$ \\
\hline $\begin{array}{l}\text { Indoor outage }(20 \\
\text { dB loss) [110] }\end{array}$ & $\begin{array}{l}10 \%(6 \mathrm{~km} \text { inter-site } \\
\text { distance (ISD); } 2.5 \% \\
\text { (4 km ISD) }\end{array}$ & $\begin{array}{l}18 \%(6 \mathrm{~km} \text { ISD }) \\
5.5 \% \text { (4 km ISD) }\end{array}$ & - & $\begin{array}{l}8 \%(6 \mathrm{~km} \text { ISD); } 1.5 \%(4 \mathrm{~km} \\
\text { ISD) }\end{array}$ \\
\hline Data rate & $\begin{array}{l}100 \quad \text { bps } \\
\text { (uplink/downlink), } \\
600 \text { bps (downlink) }\end{array}$ & $\begin{array}{l}0.25-50 \text { kbps (depen- } \\
\text { dent on BW, SF) } \\
{[111]}\end{array}$ & $\begin{array}{l}\text { 3-7 mbps (uplink); } 4 \\
\text { mbps (downlink) }\end{array}$ & $\begin{array}{l}127 \mathrm{kbps} \text { (uplink); } 159 \mathrm{kbps} \\
\text { (downlink) [Rel 14] }\end{array}$ \\
\hline
\end{tabular}

a small fraction of what the lower band technologies can tolerate, which enables seemingly impossible over-the-air latency of tens of microseconds [120]. Additionally, for industrial use cases, the large outdoor to indoor penetration losses, that eliminate legitimate interference and illegitimate jamming from outside the factory, makes the mmWave bands attractive $[121,122]$.

b) Waveform and Multiple Access Design in New Spectra: Evidently, the choice of waveform design and multipleaccess technique for mmWave deployments is still being investigated as an open problem. This owes to the differences in radio propagation as well as the hardware design challenges in high frequencies. orthogonal frequency-division multiplexing (OFDM) has long been adopted in cellular technologies, thanks to its low complexity of frequency-domain processing and high spectral efficiency. It further offers extensive flexibility in multi-user access. Impact of hardware impairments such as phase noise, non-linear power amplifiers, and analogto-digital converter (ADC) could minimize the performance. In fact, using single-carrier waveform such as single-carrier frequency domain equalization can be beneficial for mmWave [123].

Alternatively, ultra wideband (UWB) transmission using carrierless spread spectrum can offer simplicity of transceiver and ideal performance for short-range mmWave communica- tions [120]. This becomes more evident when considering use cases such as industrial wireless control, where a number of actuators need to communicate periodically with a controller in close vicinity, at sub-milliseconds cycle time. In such cases, transmission using low-power UWB can take advantage of wide mmWave bands in favor of short latency while minimizing the interference footprint.

2) Adaptive Network Topology: In the design of a large network of IoT sensors, e.g., the likes of wireless sensor networks (WSNs) in agricultural, industrial, and urban sensory networks, low deployment cost has been a key driver $[124,125]$. On the other hand, IoT devices and sensors are, for the most part, expected to be inexpensive, low-power e.g., capable of $>10$ years of continuous operation on a single battery [125]- and hence, low-range in communication reach. Inevitability, this creates a dilemma for densification of APs in network design: a low density of network APs can severely reduce deployment costs, yet, it makes it virtually impossible for all the IoT nodes to have a dependable access to the network. One can easily imagine such a dilemma in the context of IoT-enabled smart agriculture, where sensor networks are deployed over thousands of acres of space to measure temperature, humidity, nutrition and $\mathrm{PH}$ levels of soil, etc., and help farmers with improved crop efficiency at lower costs [126]. This has mainly driven wireless engineers 
to enable mesh-type of network topology for low-cost wireless IoT networks. Essentially, all the communication nodes in the network take part, not only in communicating their own data, but also in supporting the communication of other nodes' data. Several existing technologies in the unlicensed band, such as the Zigbee and LoRa networks have built upon the concept of WSN to enable low power mesh networks among lowcost sensors. Nevertheless, research and development activities in WSN are ongoing with a strong focus for utilizing D2D relaying in sensor network topologies. Particularly, fulfilling QoS requirements in a WSN has generally been studied for networks with rather homogeneous QoS across the sensors [127-131]. The future WSNs are envisioned to include a variety of sensors with rather heterogeneous QoS requirements and battery-life capabilities, which motivates an adaptive network topology design.

a) D2D Relaying for Extended Coverage and Improved Efficiency: Utilizing D2D relaying has been long studied and specified for various wireless technologies. The goal has been to extend coverage, improve the battery life of sensors, and enable out-of-coverage communication between devices [132]. For example, in the context of the 3GPP standard developments, several relay-based specifications have been made available in the past years. This includes the proximity service specifications -service for devices within close proximity of each other, especially in public safety communication servicesin Release 12 [133], and the studies on user equipment (UE) relays, self-backhauling and integrated backhauling in Release 14 and Release 15 of the specifications [134, 135]. Similarly, communication between vehicles was addressed in vehicleto-vehicle and vehicle-to-everything studies in Release 16. More recently, those attempts are extended towards enabling enhanced multi-hop relaying for extensive coverage and improved energy efficiency for Release 17 and beyond [125]. The studies in [125] offer interesting insights into the future of D2D relaying for wireless networks, including use cases such as in-home traffic, smart city, low-power wearables, and smart factories, to name a few. For instance, in the wireless deployments of the future, different facilities can be modeled as environments having variant topologies with multiple walls, long corridors, lots of metallic shelves and other static or moving objects -e.g., moving fork lifts, agricultural machines, slow-moving trains in the warehouses, and moving cranes in harbors. Meanwhile, to be able to thoroughly monitor the situation in such environments one can imagine massive wireless sensor networks with heterogeneous QoS requirement being deployed, including sensors that are installed in places with poor coverage. The argument is that mesh type of relaying will help in increasing coverage for these low-power sensors, which are expected to also have a long battery life.

b) Cooperative D2D Relaying for Reliability: In the context of industrial automation, a similar point on the benefits of network-device cooperation can be made, although from a different perspective. In such scenarios, actuators of the same or different production machines communicate together under extreme reliability and latency requirements, while co-existing with a large number of sensors that monitor the production process and periodically reporting sensory information to update the controller about the state of the machine [136138]. Overall, sensors and actuators in an industrial production line are under much more severe scrutiny -compared to, e.g., agricultural sensor networks- with regard to reliability and timeliness (a.k.a, latency) of their communication with the production line controller. On the other hand, the communication range is not an issue in such deployments with nodes typically being in close vicinity of the controller AP. The challenge is however to guarantee reliability at five nine figure (i.e., $99.999 \%$ of the time) and higher, up to nine nines [120]. Therefore, it is vital to exploit the available sources of diversity in the network in favor of link reliability. Aside from the limited amount of time and frequency diversity -limited by physics of the environment in form of coherence time and frequency- the network should exploit spatial diversity and multi-path diversity through cooperation among the nodes $[139,140]$. In fact, it is shown that relying solely on frequency diversity to achieve $10^{-9}$ error rate requires an impractically high signal-to-noise ratio (SNR) values in realistic channel conditions [139].

Spatial diversity in the form of multiple antennas at the transmitter and receiver sides is a promising way to collect diversity gain. This has been widely studied in the literature around MIMO [141]. Such solutions are powerful in transforming the harsh distribution of typical fading channels (e.g., Rayleigh distribution) into more benign distributions where the channel is most of the time above the detection threshold. In fact, with the increasing deployment of massive MIMO, in practice, the impact of fast fading can be largely under control, hence, cellular communication technologies typically rely on the channel hardening effect of MIMO to mitigate the effect of small-scale fading [142]. However, the largescale variations in the channel, particularly in the highly dynamic industrial environments, can cause severe challenges for reliable communication system design. For example, the large size (relative to wavelength) moving machines and cranes in an industrial environment can create a shadowing effect by blocking a line-of-sight link, or, completely change the propagation characteristics of the environment by creating strongly reflected paths that could cause both constructive and destructive impact on link signal strength.

c) Adaptive Network-Device Cooperation: For the above-mentioned reasons, it is highly favorable to adopt cooperation among devices in the network in form of $\mathrm{D} 2 \mathrm{D}$ relaying and multi-hop transmission, which unleashes an abundant source of spatial diversity in favor of reliability. In essence, for a network with a large number of devices to be served, the devices with a strong momentary link to the controller AP can communicate directly with the AP, while the other devices with weak link quality must be served by a cooperative transmission from the AP and the strong devices. Such a technique was first introduced in [139]. Although the analysis in [139] was focused on mitigating the impact of fast fading, it motivated the use of cooperative D2D relaying for ultrareliable applications by demonstrating $10^{-9}$ system probability of error-i.e., the probability of at least one device failing to receive a downlink packet or delivering an uplink packet in each cycle time-for a network with 30 devices, at practical 
SNR value of $5 \mathrm{~dB}$. Interestingly, such reliability level was achieved without CSI at the transmitter side, therefore, not relying on link-adaptation and pre-coding. On the other hand, such cooperative transmission techniques utilize several relays to forward a message to a weak device, thus, inevitably, increasing the interference footprint of each cell [143]. More importantly, without the use of CSI, radio resources are inefficiently exploited, resulting in loss of spectral efficiency [144]. Other techniques in the literature are proposed to utilize the partial knowledge of CSI at the transmitter, e.g., in the form of channel quality indicator (CQI), to identify devices with weak channel conditions and to deploy the cooperative relaying resources in an on-demand fashion, only for the devices with momentary poor coverage. Channel-awareness for allocating resources for each transmission and enabling ondemand cooperative relaying is the advent of techniques with adaptive network-device cooperation [144]. This increases the spectral efficiency while improving the system-level reliability by reducing the interference footprint of the relaying process.

\section{Channel Code Design}

As discussed in Section II-A, the next generation of IoT systems need to guarantee various service requirements in terms of latency and reliability. This has posed challenges in the design and implementation of channel coding techniques, which have been previously designed and optimized for long block lengths, mostly used for human-based communications. Interested readers are referred to [12] for a detailed discussion on the PHY design for wide-area IoT, which includes but is not limited to characteristics and the scheduling of downlink and uplink physical channels at the NB-IoT base station side and the user equipment side. In what follows, we summarize the channel coding challenges for IoT systems.

- Design of short block length codes: In many IoT applications, devices send only a small packet of data in a sporadic manner. The payload data could be very short, ranging from only a few to up to a few hundred bits. On the other hand, to guarantee the delay requirement of delay-sensitive applications, it is necessary to use shorter packets and accordingly shorter time-to-transmit intervals. However, if the block length decreases, the coding gain will be reduced and the gap to Shannon's limit will increase. This is mainly due to the reduction in channel observations that comes with finite block lengths and is not due to the channel code. Moreover, most of the existing codes with efficient decoders, such as LDPC or Turbo codes, show a gap to finite length bounds. This is often due to the suboptimal decoding algorithms.

- Design of efficient decoding algorithms: Many IoT applications demand ultra-low latency. Despite the improvement of the round-trip delay from around $15 \mathrm{~ms}$ in $4 \mathrm{G}$ to around $50 \mu \mathrm{s}$ in $5 \mathrm{G}$, the receiver components, including the decoder, still require significant improvement in terms of delay to meet the latency requirement. For example, the successive cancellation decoding technique of polar codes imposes a significant delay due to the successive nature of the decoder. Parallel decoding techniques, such as the belief propagation algorithm, are favorable, but they work well for only sparse codes on the graph, such as LDPC codes.

- Achieving lower block error rates: The design target for channel code in the LTE standard was to achieve the block error rate (BLER) of $10^{-2}$, however, many IoT applications require high reliability. That means that the BLER should be less than $10^{-5}$ and even lower than $10^{-9}$ for industrial IoT. Achieving such a low BLER with efficient decoders is very challenging, especially when we want to perform as close as possible to the finite-length bounds. Some codes, like LDPC codes, show error-floor under practical decoders, which limit their performance for low-latency applications.

- 1-bit granularity in the codeword length and rate should be maintained for channel codes. This is essential for enabling retransmission techniques and achieving low code rates. Accordingly, efficient error detection algorithms should be developed paired with modern MAC protocols.

\section{A. State-of-the-Art Channel Coding Techniques for IoT}

Several channel coding techniques have been considered for IoT. The main requirements are for a low-complexity encoder and decoder with shorter block size information and low modulation orders to satisfy low-power requirements. Candidate channel coding techniques for IoT are polar code, LDPC code, Turbo code, and tail-biting convolutional code (TBCC).

1) Polar codes: The Polar code was introduced by Arikan in 2008 [145] and was recently standardized for the eMBB control channel in 5G new radio (NR). The code can achieve the capacity of the binary-input memoryless channel under low-complexity successive cancellation (SC) decoding. The polar code can be realized as a recursive concatenation of a base short block code designed to transform the transmission channel into a set of virtual channels with different levels of reliability. The information bits are put only into the reliable channels and foreknown bits into the unreliable channels, also referred to as Frozen Set. The encoder is through polarization which is given by the kernel $T_{2}=\left(\begin{array}{ll}1 & 0 \\ 1 & 1\end{array}\right)$. For longer inputs, the transformation is obtained through the Kronecker product of $T_{2}$ with itself. Fig. 10a shows the polar encoder of length 4 . The decoder sets all frozen bits to zero, and starts the decoding of information bits from the highest reliable channel in a successive manner.

2) LDPC codes: The LDPC code was first proposed by R. G. Gallagher [146] in 1962 and rediscovered later in 1997 [147], when a practical iterative decoder based on the belief propagation (BP) was designed. LDPC codes under the BP decoding have shown to perform very close to Shannon's limit [148]. An LDPC code is mainly characterized by its sparse parity check matrix (also shown as Tanner graph in Fig. $10 \mathrm{~b}$ ), which facilitates low complexity decoding. The design of LDPC codes is mainly to find the degree of nodes in the Tanner graph and then construct the parity check matrix in such a way to avoid short cycles. The BP decoding involves exchanging beliefs (most commonly log-likelihood ratios) between nodes 


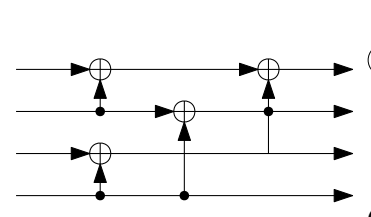

(a) Polar encoder of length 4.

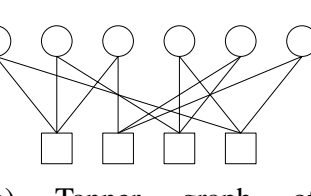

(b) Tanner graph of low-density parity-check (LDPC) code.

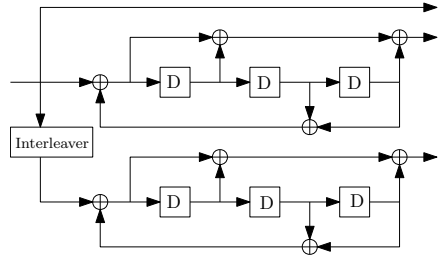

(c) Turbo code encoder.

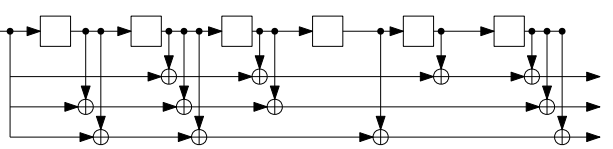

(d) TBCC encoder.

Fig. 10: The encoder of channel code candidates for IoT.

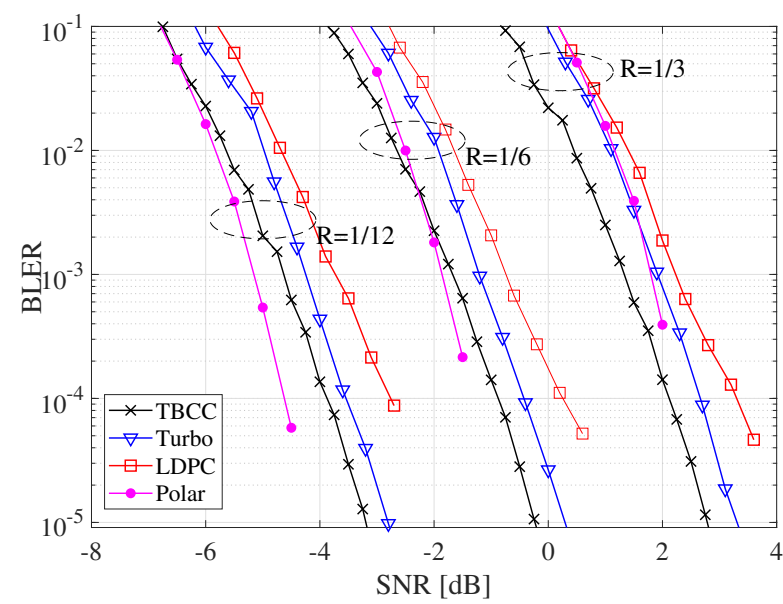

(a) Very short information block length, $k=40$.

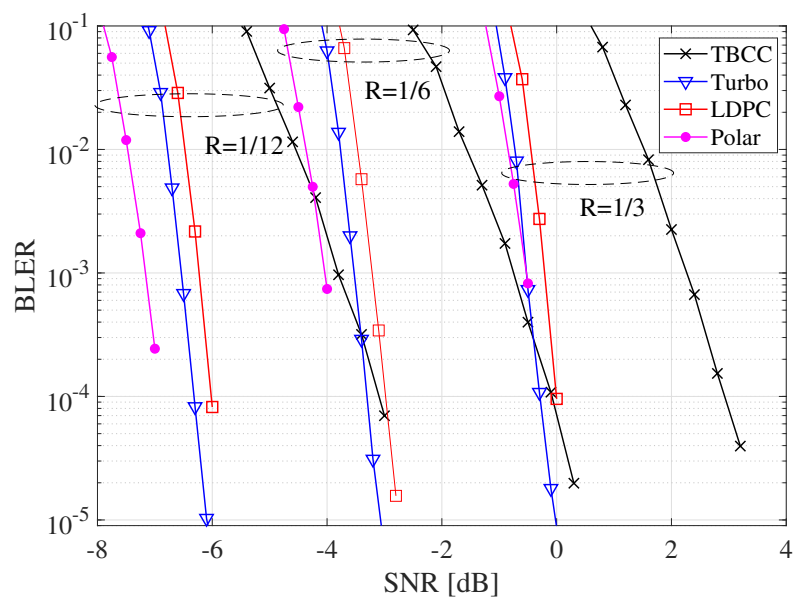

(b) Moderate information block length, $k=600$.

Fig. 11: BLER performance of candidate channel codes for IoT. Turbo and TBCC codes are taken from the LTE standard. For polar code, cyclic redundancy check (CRC) was added and the list size 32 was used for decoding. For LDPC, the layered offset min-sum decoder was used with the maximum iteration number of 25 .

along the edges of the Tanner graph in an iterative manner until convergence. A variant of LDPC codes, called the protographbased Raptor-like LDPC code, which is a concatenation of a high-rate LDPC code and a low-density generator matrix code, has been recently standardized for the $5 \mathrm{G}$ eMBB data channel due to their excellent performance at moderate-to-long block lengths and their low-complexity decoding. These codes however do not perform well in the short block length regime and show an error floor, which limits their application for short packet communications with latency and reliability constraints [149].

3) Turbo codes: Turbo codes were proposed in 1990 and were adopted in the data channel in LTE mobile communications. It is also the channel coding technique for NB-IoT. The turbo code is usually a parallel concatenation of two recursive convolutional encoders (see Fig. 10c), which are separated by an interleaver. The decoder of Turbo codes runs in an iterative manner by exchanging extrinsic information between two constituent decoders. The Turbo code with the iterative decoder is capable of performing within a few tenths of $\mathrm{dB}$ from Shannon's limit. However, it shows a gap to the finite length bound at short and moderate block lengths. Moreover, when the code rate is below $1 / 3$, the performance is further degraded. One advantage of Turbo codes is the capability to support 1-bit granularity for all coding rates and for a full range of block sizes.
4) TBCCs: TBCC has been used in the control channel of the LTE and NB-IoT data channel due to its low complexity encoding and decoding and outstanding performance at short block lengths. The encoder of a TBCC is shown in Fig. 10d. Unlike convolutional codes which require some termination bits to force all the states to the zero state, TBCC does not terminate states to zero, therefore achieving better rates. The decoder relies on the Viterbi decoder which estimates the maximum likelihood sequence using the code trellis.

Figure 11 shows the BLER comparison between candidate channel codes for IoT. As can be seen, TBCC outperforms other codes at very short block lengths (Fig. 11a) however its performance degrades at lower code rates. Polar codes show excellent error performance at both short and moderate block lengths without any error floor. However, they suffer from relatively high-complexity list successive cancellation decoder. The decoder also introduces significant delay which might not be tolerable for delay-sensitive applications. Turbo codes which are being used in LTE and NB-IoT are performing reasonably well at moderate block lengths, which suggests that they can be a favorable candidate due to their fast and very-low complexity decoders.

\section{B. Low-capacity Communications}

The current strategies for serving a massive number of IoT are mainly based on narrowband technologies. For example, NB-IoT will serve up to 50,000 mMTC devices in one cell and 
mMTC devices in very remote areas. The effective channel SNR can be as low as $-13 \mathrm{~dB}$ or with a capacity 0.03 [150]. In other words, the communication is over a very low-capacity channel. Another scenario is wideband communications, e.g., millimeter-wave in 5G and Terra-Hertz in 6G, which have higher frequencies and much larger bandwidths. Under a constant power, we will have a higher data rate but the individual bits will be going through channels with very small SNR [150]. Current techniques, such as repetition (in NBIoT) and HARQ with fixed-rate channel codes are inefficient in these scenarios.

Authors in [150] showed that concatenating the state-ofthe art codes at moderate rates with repetition codes may be a practical solution for various applications, concerning ultra-low power devices. A polar-coded repetition strategy was also proposed with the aim to limit the encoding and decoding complexity. In [151], Raptor codes were designed for very low SNR channels, where the degree distribution was optimized to achieve the rates efficiencies as high as $96 \%$. The codes are however, mainly designed and optimized for asymptotically long block lengths and their performance dramatically degrade at short blocklengths. The decoder of Raptor codes at low SNRs also requires a very large number of iterations to converges, which might not be tolerable for applications with latency constraints.

The decoding in low-capacity communications is also challenging due to the fact that the received symbols are not reliable. In particular, the log-likelihood ratios (LLRs) are very small, that is the iterative message passing decoders, in case of LPDC or Raptor codes, require a large number of iterations to converge. Moreover, the successive interference cancellation even with large list sizes may not perform well when the LLRs are very low. Increasing the list size significantly increases the decoding complexity which is not feasible for latencyconstrained scenarios.

\section{Channel Coding for CSI-free Communications}

Most of existing channel adaptation techniques rely on the CSI usually sent from the receiver to the transmitter via a feedback message. In adaptive modulation and coding (AMC) the transmitter chooses a fixed-rate channel code with a proper rate and the modulation scheme in order to maximize the spectral efficiency according to the CSI. There are three main problems with this approach which limits its efficiency in future IoT applications. First, obtaining CSI to all IoT devices is not practical especially in massive IoT scenarios with sporadic traffic. Second, even if the CSI could be obtained for IoT devices, it is not practical to frequently update it due to random traffic, mobility, and activity of devices. Third, obtaining CSI requires the transmitter to send pilot sequences to the receiver and then receive the CSI via a feedback message. This is time-consuming and adds significant overhead which will be further challenging in massive IoT scenarios with a very large number of devices. Moreover, this overhead and the delay associated with obtaining CSI in most cases violate the latency requirements of many delay-sensitive applications [152].

Retransmission techniques can be used to adapt to the channel condition without the CSI at the transmitter side.

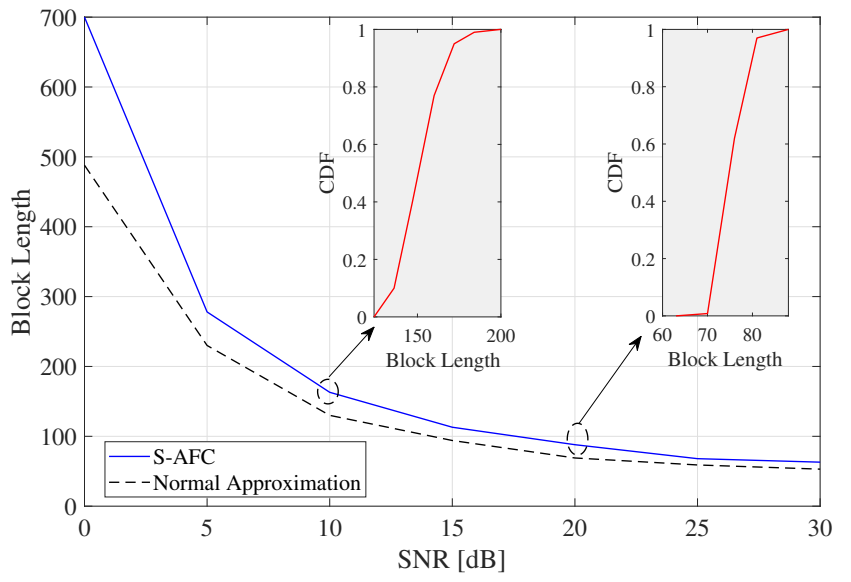

Fig. 12: Average block lengths of AFC versus SNR. A $(256,192)$ LDPC code was used as the precode for AFC and the target BLER is $\epsilon=10^{-4}$.

In particular, HARQ can achieve a fine-grain matching for channel conditions with multiple retransmissions and acknowledgments. However, these approaches are mainly effective when channel variations are slow. Severe path loss, high blockage and fluctuating characteristics of wireless channels at mm-Wave, and higher frequency bands which are expected to be used for the 5G and beyond of mobile standards [152], have questioned the suitability of current adaptive transmission techniques to meet the reliability and latency goals of future networks.

Rateless codes have gained interests in recent years due to their automatic channel adaptation features [149]. Most of these codes have been designed for long message lengths and the encoding mostly involves random generations of parity symbols. Examples include Raptor codes [153], Strider [154], rate compatible modulation (RCM) [155], analog fountain code (AFC) [156], and Spinal codes [157]. The aim is to achieve a fine granularity of spectral efficiency and seamless link adaptation without the feedback of channel condition. Although several attempts [158-162] have been made to optimize these codes for short message lengths, the codes still suffer gaps to finite block length bounds, such as the normal approximation bound [163], when practical decoders with bounded complexity are being used [149]. Fig. 12 shows the performance of short AFC [158] in terms of the average block length to achieve a target BLER of $10^{-4}$. As can be seen, this code performs close to the normal approximation bound in a wide range of SNRs, however, further improvement at low SNRs is required. It is also important to note that at low SNRs the block length required to achieve the desired level of BLER varies significantly, which might limit the use of the code for delay-sensitive applications.

BCH-DFT codes [166-168] are another class of analog codes that have been applied in various applications in channel coding, joint source-channel coding, and distributed source coding $[170,172,173]$. These codes can be used for erasure detection too [174-176]. Classical decoding for this class of codes is called the coding-theoretic approach [168]. To be 
TABLE VI: Comparison of channel code candidates for IoT applications.

\begin{tabular}{|c|c|c|c|}
\hline Code & Decoding & Advantages & Disadvantages \\
\hline LDPC codes & $\begin{array}{l}\text { Belief propagation de- } \\
\text { coding, min-sum de- } \\
\text { coder, max-sum de- } \\
\text { coder }\end{array}$ & $\begin{array}{l}\text { Excellent performance at long-block } \\
\text { lengths, efficient decoder, parrallelization } \\
\text { of the decoder to reduce latency }\end{array}$ & $\begin{array}{l}\text { Error floor at short and moderate block } \\
\text { lengths, the BP decoder is suboptimal for } \\
\text { short codes, 1-bit granularity cannot be eas- } \\
\text { ily achieved without loosing performance }\end{array}$ \\
\hline Polar codes & $\begin{array}{lr}\begin{array}{l}\text { Successive } \\
\text { cancellation, }\end{array} & \text { List } \\
\text { decoding, } & \text { Stack } \\
\text { decoding, } & \text { Belief } \\
\text { propagation } & \end{array}$ & $\begin{array}{l}\text { Can achieve very low BLER, No sign of er- } \\
\text { ror floor, 1-bit granularity can be achieved, }\end{array}$ & $\begin{array}{l}\text { The successive decoder introduces delay, } \\
\text { the list decoding is complex and not prac- } \\
\text { tical for low-power devices, Large list size } \\
\text { should be used to achieve very low BLER }\end{array}$ \\
\hline Turbo codes & Iterative soft decoding & $\begin{array}{l}\text { Very fast encoder and decoder, Parallel ar- } \\
\text { chitecture can be used to boost the decod- } \\
\text { ing speed, 1-bit granularity can be easily } \\
\text { achieved, close-to-Shannon performance at } \\
\text { large block lengths }\end{array}$ & $\begin{array}{l}\text { Loose performance at short and moderate } \\
\text { block lengths, Large gap to the finite-length } \\
\text { bounds as short block lengths }\end{array}$ \\
\hline TBCC & Viterbi decoding & $\begin{array}{l}\text { Fast encoder and decoder, can achieve very } \\
\text { low BLER at very short block lengths }\end{array}$ & $\begin{array}{l}\text { The performance degrades at moderate } \\
\text { block lengths or rates lower than } 1 / 3 \text {, large } \\
\text { memory order should be used to achieve } \\
\text { lower BLER, The decoding complexity ex- } \\
\text { ponentially increases with the memory order }\end{array}$ \\
\hline $\begin{array}{l}\text { Algebraic codes } \\
\text { (Hamming codes, } \\
\text { Golay codes, Reed- } \\
\text { Muller codes, } \\
\text { Bose-Chaudhuri- } \\
\text { Hocquenghem } \\
\text { (BCH) codes, Reed- } \\
\text { Solomon codes) }\end{array}$ & $\begin{array}{l}\text { Ordered statistics } \\
\text { decoder (OSD) [164], } \\
\text { Chase decoding, } \\
\text { Berlekamp-Massey } \\
\text { decoding, majority- } \\
\text { logic decoding, } \\
\text { maximum-likelihood } \\
\text { decoding }\end{array}$ & $\begin{array}{l}\text { Fast encoders, large minimum Hamming } \\
\text { distance, can achieve very low BLER with- } \\
\text { out an error floor }\end{array}$ & $\begin{array}{l}\text { The decoding is very complex, the decoding } \\
\text { performance degrades when low-complexity } \\
\text { decoders are used, OSD can achieve near } \\
\text { ML performance but the complexity is huge } \\
\text { especially at low rates and moderate block } \\
\text { lengths }\end{array}$ \\
\hline $\begin{array}{l}\text { Fountain codes } \\
\text { (Raptor code [153]) }\end{array}$ & $\begin{array}{l}\text { Message-passing } \\
\text { decoding }\end{array}$ & $\begin{array}{l}\text { Codes can automatically adapt to the chan- } \\
\text { nel without channel state information, the } \\
\text { message passing decoder can be imple- } \\
\text { mented efficiently }\end{array}$ & $\begin{array}{l}\text { The code performs well when the block } \\
\text { length is very large, the code has been } \\
\text { optimized for erasure channels and over } \\
\text { wireless channels the code design is channel } \\
\text { dependent, Codes do not perform well at } \\
\text { short block lengths, the decoding complex- } \\
\text { ity is large at low code rates }\end{array}$ \\
\hline $\begin{array}{l}\text { Rate Adaptive codes } \\
\text { (AFC [165], RCM } \\
\text { [155], Spinal [157]) }\end{array}$ & $\begin{array}{l}\text { BP for AFC and RCM, } \\
\text { and Viterbi-like decod- } \\
\text { ing for Spinal codes }\end{array}$ & $\begin{array}{l}\text { Can adapt to the channel condition auto- } \\
\text { matically without channel state information, } \\
\text { can be optimized for short block lengths, } \\
\text { achieve the finite-length bound over a wide } \\
\text { range of SNRs, iterative belief propagation } \\
\text { decoding can be applied (for AFC and } \\
\text { RCM) }\end{array}$ & $\begin{array}{l}\text { The decoding complexity can be large, the } \\
\text { output signal are usually from large constel- } \\
\text { lations }\end{array}$ \\
\hline 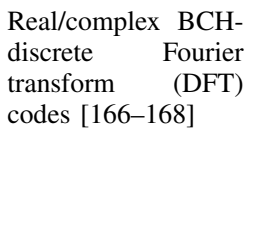 & $\begin{array}{l}\text { Coding-theoretic } \\
\text { approach }[168,169] \\
\text { and subspace-based } \\
\text { approach }[170,171]\end{array}$ & $\begin{array}{l}\text { Improved performance as code length re- } \\
\text { duces. Can be used for channel coding, } \\
\text { joint source-channel coding, and distributed } \\
\text { source coding }[170,172,173] \text {, good perfor- } \\
\text { mance for erasure detection }[174-176]\end{array}$ & $\begin{array}{l}\text { To be used in digital communication sys- } \\
\text { tems, the output of this class of codes should } \\
\text { be quantized and converted to bits. Both } \\
\text { decoding algorithms are sensitive to quan- } \\
\text { tization error and the performance drops if } \\
\text { the number of quantization levels is not big } \\
\text { enough. }\end{array}$ \\
\hline
\end{tabular}

able to use these codes in digital communications, the codewords undergo a quantization process. This process introduces quantization error to each element of the codeword. This, in turn, deteriorates the decoding performance. Subspacebased method $[170,171]$ is an alternative decoding approach that is more robust to the quantization error. The decoding performance still may not be acceptable when the number of quantization levels is small or the code length is large. As such, these codes are more useful for short block length. The drawback of the short block length is that the code capacity move away from Shannon limits.

\section{Decoder Design}

Although algebraic codes, such as $\mathrm{BCH}$ and Reed-Solomon codes, have large minimum Hamming distance and ac- cordingly excellent BLER performance with the maximumlikelihood (ML) decoding, their decoding complexity has made them impractical for IoT applications. On one hand, most of the near-ML decoding algorithms, such as the OSD [177], are very complex and even their best implementations introduce significant latency. On the other hand, their implementations are not power-efficient, therefore cannot be embedded into low-power devices. Researchers have recently proposed several approaches to reduce the complexity of the OSD algorithm, which are mainly focused on reducing the number of test error patterns when searching for the best candidate codeword. In particular, sufficient and necessary conditions have been introduced to terminate the decoding early or skip the test patterns without encoding them [178, 179]. There are still however issues that need to be resolved. 
First, although these approaches can significantly reduce the average decoding complexity, the worst-case scenario is still very complex and the receiver might need to search through a large number of test patterns. Second, most of the OSDbased approaches run in iterations and it seems hard to devise efficient algorithms for parallelization of the process. However, approaches like segmentation-discarding [180] and efficient tree-based search algorithms [181] might help to further reduce the complexity.

The BP decoding algorithm of LDPC codes involves a complex non-linear function in the check-node processing. This leads to large implementation complexity. Several simplified alternative algorithms, like min-sum [182], normalized minsum [183], and off-set min-sum [183], have been proposed and being widely used in practice thanks to their inherent parallelism for hardware implementation. The simplification however leads to a significant performance loss, especially at low rates, short-to-moderate block lengths, and when the decoding iteration number must be kept low, which are the scenarios of interest for IoT [184]. Moreover, the 5G LDPC codes have numerous degree- 1 variable nodes, which are very prone to be erroneous [185]. Several approaches have been proposed in the literature to improve the decoding complexity of 5G LDPC codes. These includes, linear-approximated BP [186], adapted min-sum [187], and improved adapted min-sum [185]. Further reduction of the complexity is necessary for the decoder of the LDPC to make them a realistic solution for IoT applications.

Polar codes can be efficiently decoded using SC decoding, however the performance significantly degrades at moderateto-short block lengths. To solve this problem, the SC List decoding [188] can be used which improved the decoding performance but also scale the complexity by a factor $M$, which is the list size. For 5G Polar codes usually a large list size, larger than 32, should be used to achieve BLER of less than $10^{-4}$. The stack SC decoding [189] has been also proposed to improve the decoding performance, which works similar to the Viterbi decoder. A hybrid list and stack SC decoding was also proposed in [190], which slightly reduces the complexity. The performance however degrades when stack-size is short. Further improvement in the decoding performance can be obtained by using CRC bits [191], which also increases the complexity. The serial nature of the SC decoding limits the speed of the decoder, which has implications for low-latency communications.

The decoders for polar and LDPC codes require the channel state information at the receiver side. When such information is not available, the decoding fails. The design of algorithms capable of handling limited or no CSI at the receiver is therefore essential for IoT applications [192]. Furthermore, the current error detection schemes are mainly based on CRC codes, which adds significant overhead when short packets of data needs to be sent. For massive IoT applications, the design of strong error detection algorithms without CRC bits is required [192].

Table VI shows a comparison between different channel coding techniques for IoT applications.

\section{Massive Connectivity}

As explained Sec. II-E, 5G and 6G networks are expected to support $10^{6}$ and $10^{7}$ devices $/ \mathrm{km}^{2}$, respectively. A big percentage of the new connections will be due to massive IoT applications, i.e., the mMTC use case. We also indicated that traffic generated by such devices is sporadic and has a small payload which is different from those of eMBB and URLLC applications. Further, mMTC devices have limited power while they may need to reach a very far BS (e.g., smart agriculture sensors which are typically deployed in rural areas). There is also a fast-growing number of IoT devices in eMBB and URLLC applications.

Considering all use cases, a range of solutions are proposed to address the diverse range of devices connecting to $5 \mathrm{G}$ and beyond networks. One popular trend is moving from orthogonal to non-orthogonal design in the waveform, random access, and multiple access designs [193]. In particular, nonorthogonal multiple access (NOMA), non-orthogonal random access, and non-orthogonal waveform design have been extensively studied during the past several years. All in all, massive connectivity solutions can be categorized as

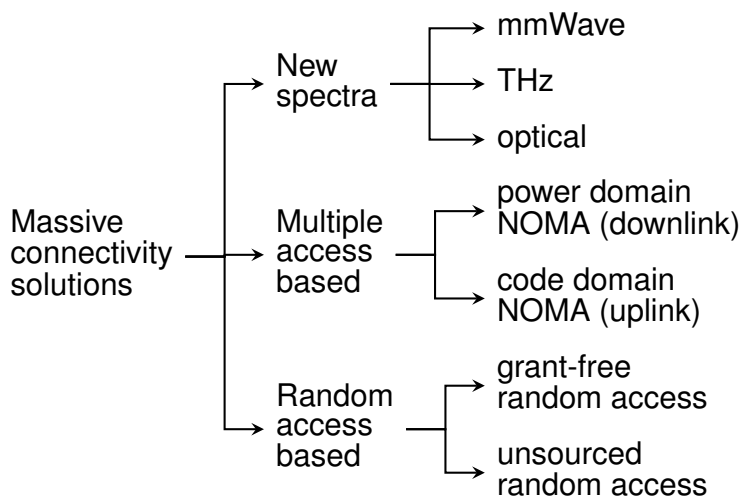

and

we are going to elaborate focus on multiple access and random access in this section.

\section{A. Multiple Access Solutions}

Existing cellular networks, including 4G LTE, have been designed based on non-overlapping radio resource allocation techniques. For example, in LTE networks, a resource block (RB) which is $180 \mathrm{kHz}$, cannot be allocated to more than one user, i.e., it cannot be shared with other users. This approach in resource allocation has two major drawbacks in massive IoT: 1) with exploding number of massive IoT is it not affordable to allocate one dedicated RB to each device, 2) massive IoT users do not deplete one RB and, thus, such resource allocation may not be efficient. In addition, IoT communication is uplinkdominated and uplink communication of massive low-rate IoT devices requires a very different set of technologies than those designed to serve humancentric communications in $4 \mathrm{G}$ and previous generations of cellular networks.

Due to the above issues and the fact that an unprecedented number of new IoT devices are projected to be connected to 
wireless networks ${ }^{5}$, the trend in communication system design has recently changed from orthogonal to non-orthogonal. This paradigm shift is happening in various MAC and PHY technologies such as waveform design, multiple access, and random access [193]. In particular, NOMA techniques, unlike their orthogonal counterparts, revolve around letting two or more users share the same RB. Hence, NOMA is a promising approach to accommodate upcoming users, in general, and IoT users, in specific.

NOMA techniques are inherently different for uplink and downlink communications. The difference becomes noticeable when it comes to IoT communication, where a massive number of uncoordinated devices transmit small packets with low data rates in the uplink. It should be noted that the downlink is mostly focused on serving humancentric communications, which typically requires large packets and high data rates. In the following, we discuss the uplink and downlink NOMA techniques.

1) Uplink NOMA: In the uplink, NOMA embraces a variety of non-orthogonal transmission techniques, These schemes are devided into power-domain NOMA and code-domain NOMA $[193,195]$. Power-domain NOMA is another name for Shannon capacity-achieving successive interference cancellation (SIC)-based coding for the multiple access channel. Examples of power-domain uplink NOMA can be found in [196-199] Code-domain NOMA, however, embraces a number of new techniques which will be discussed in the following.

Transmitter Structure: Resource overloading is the common theme of these non-orthogonal access methods which allows the number of supportable users to be more than the number of available resources. Then, a NOMA scheme needs a signature to differentiate users and cancel inter-user interference. The structure of a generic NOMA transmitter is depicted in Fig. 13 $[195,200]$. Different NOMA schemes apply their signatures in one or more of the dashed blocks in Fig. 13 [195, 200, 201].

Categories/Schemes: Code-domain NOMA schemes can be categorized in various ways. As can be seen in Fig. 13, NOMA-related operations can be done in the bit-level (before modulator) or system-level. For this reason, as also shown in Fig. 14, NOMA schemes may be categorized as follows:

- Bit-level interleaving/scrambling: An interleaver or a scrambler at bit-level is used to distinguish the users in this case [202]. A bit-level scrambler incurs less processing delay and memory requirement compared to bit-level interleaver [195]. Examples of this category are interleave division multiple access (IDMA) [202], asynchronous coded multiple access (ACMA) [203], and low code rate spreading (LCRS) [204]. Some NOMA schemes apply more than one of the above methods. For example, interleave-grid multiple access (IGMA) [205] uses both bit-level interleaving and sparse mapping.

- Symbol-level spreading: The majority of NOMA schemes fall in this category where a spreading sequence is used as a signature to distinguish the users. Notable

\footnotetext{
${ }^{5}$ Cisco visual networking index projects about $20 \%$ annual growth in the machine to machine connections until 2021 [194]. Ericsson 2018 mobility report forecasts 30\% annual growth in IoT devices between 2017 and 2023 [6].
}

examples are: sparse code multiple access (SCMA) [206], non-orthogonal coded access (NOCA) [207], nonorthogonal coded multiple access (NCMA) [208], multiuser shared access (MUSA) [209], low-density signature (LDS) [210,211], and pattern division multiple access (PDMA) [212].

- Symbol-level interleaving/scrambling: A symbol-lever interleaver/scrambler is used to distinguish the users. Low code rate and signature-based shared access [213], resource spread multiple access (RSMA) [214,215], and repetition division multiple access (RDMA) [216] are examples of this category.

Symbol-level spreading-based NOMA schemes are mostly inspired by code division multiple access (CDMA). CDMA is a multiple access technique in which data symbols are spread over a group of user-specific mutually-orthogonal codes. LDS is a version of CDMA in which spreading codes have a low density, i.e., a small fraction of code's elements are non-zero [210,211]. This allows using near-optimal message passing algorithm (MPA) receivers with practically feasible complexity. In spite of its moderate detection complexity, LDS suffers from performance degradation for constellation sizes larger than QPSK.

SCMA is a multi-dimensional codebook NOMA scheme in which the encoder directly maps incoming data bits to SCMA codewords selected from a layer-specific codebook where each codeword represents a spread transmission layer [234]. While in CDMA (and LDS) spreading and bit to symbol mapping are done separately, SCMA merges these two steps and directly maps incoming bits into a spread codeword within the SCMA codebook sets.

Due to the sparsity of codewords, like LDS, SCMA enjoys the low complexity reception techniques [219]. Additionally, it takes advantage of additional degrees of freedom in the design of multi-dimensional constellations and outperforms LDS [234]. More importantly, sparser SCMA codewords can tolerate more overloading to enable massive IoT connectivity in the uplink. A sparser code, however, will result in lower coding gain. SCMA can also be used for the downlink [206, $217,218]$, but the complexity decoding is still very high for low-cost IoT devices and this limits the capability of SCMA to support massive IoT connectivity in the downlink [218].

Table VII compares the complexity and overloading factor for a selected group of the above non-orthogonal multiple access (NOMA) schemes listed in Fig. 14. The schemes with higher overloading and lower complexity are preferred to others.

2) Downlink NOMA: In the downlink, NOMA reaps the benefits of the broadcast channel (BC) in which all users can transmit their messages at the same RB [222-224]. In a single-cell, single-input and single-output (SISO) system, such transmission is theoretically optimal and superposition coding is used at the BS and SIC is used to cancel the interuser interference introduced by all users with weaker channel gains while treating the interference of stronger users as noise. As such, all users except the one with the weakest channel gain will need to apply SIC for decoding. Although SIC has been implemented in smartphone size devices [235], it is too 


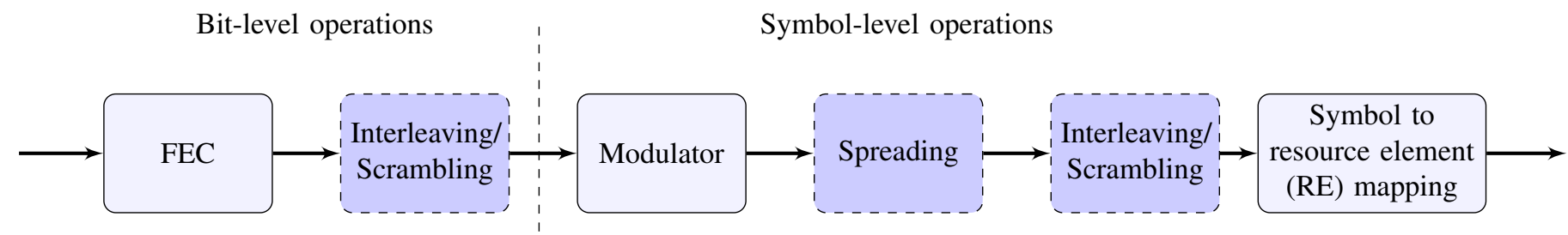

Fig. 13: General channel structure of a NOMA transmitter. Each NOMA scheme may have one or more of the dashed blocks.

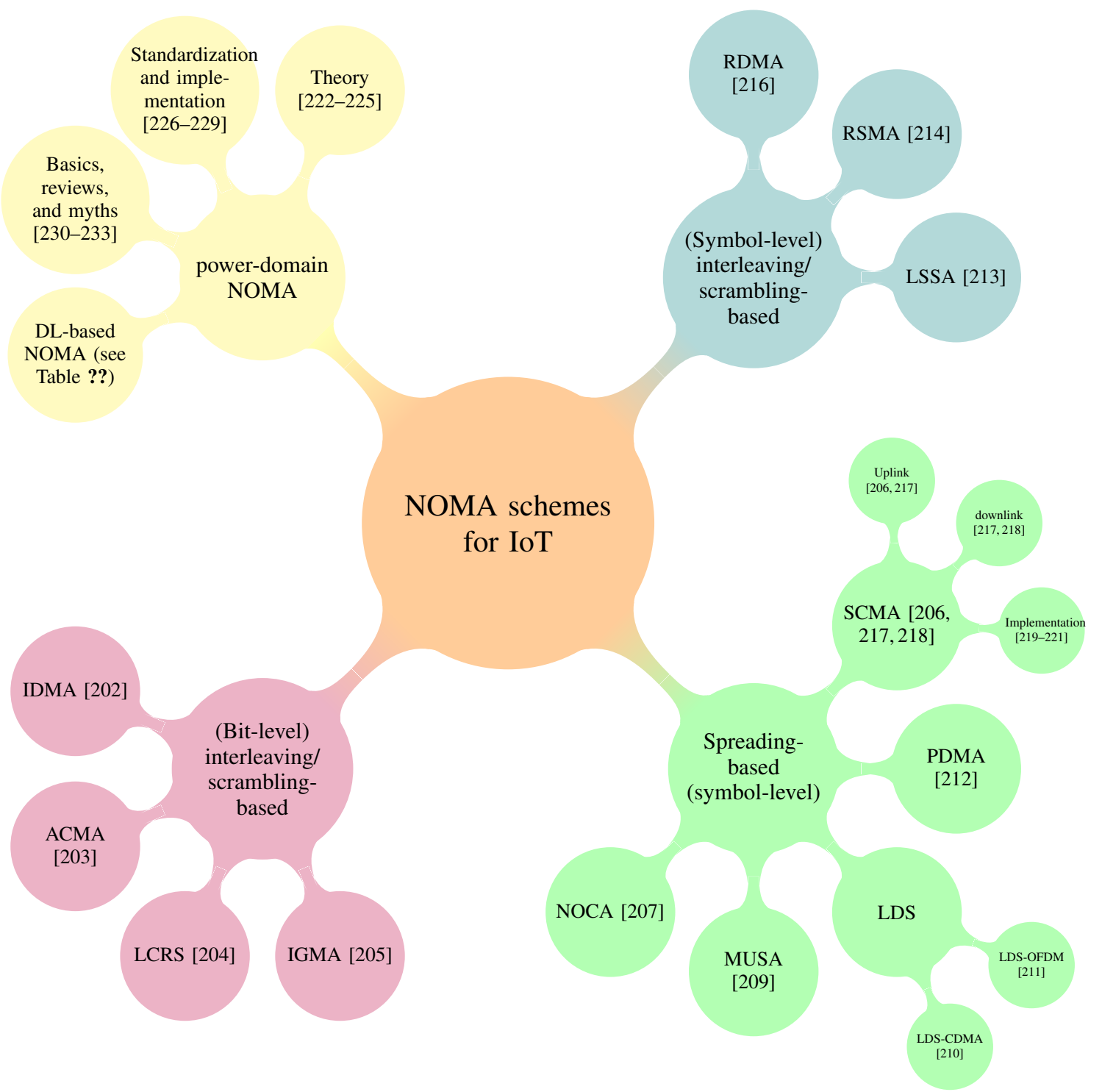

Fig. 14: Various NOMA schemes proposed in 3GPP 5G RAN meetings. Code-domain NOMA (mostly uplink) is categorized into scrambling-based, spreading-based, interleaving-based, hybrid, and others. Power-domain NOMA (downlink) is in a separate category. 
TABLE VII: Complexity and overloading for selected code-domain NOMA schemes

\begin{tabular}{llllllll}
\hline & SCAM & IDMA & MUSA & IGMA & PDMA & NOCA & RSMA \\
\hline Proposing company & Huawei & InterDigital & ZTE & Samsung & CATT & Nokia & Qualcomm \\
Receiver complexity & High & Moderate & Low & High & High & Low & Low \\
Overloading factor & Moderate & High & High & High & Moderate & Moderate & High \\
\hline
\end{tabular}

complex for simple IoT devices. As a result, although some papers propose downlink NOMA for IoT users [236-240], due to the SIC complexity, practically it is hard to justify more than one IoT device in each group of NOMA. This may limit the large-scale implementation of NOMA for IoT devices.

In Release 13 and 14 LTE, multi-user superposition transmission (MUST) was studied and specified as a downlink NOMA scheme [226]. However, MUST was not borrowed for 5 G NR downlink since the gain from NOMA was marginal in massive MIMO setting, and the study was terminated in October 2016 [200]. As a result, Release 15 NR networks are largely OMA-based. Also, the fact that power-domain NOMA requires a relatively complex receiver, the application of NOMA to IoT networks is not trivial and needs careful studies. With the recent trend of applying deep learning (DL) to communication systems, in general, and to NOMA [5, 241243], in specific, the chance of coming up with viable NOMA solutions for IoT networks increases. As an example, in [244], it is shown that DL-based precoding is advantageous in terms of delay and complexity and is more suitable for applications.

It is worth highlighting that superposition coding-successive interference cancellation (SC-SIC) is not an optimal solution when nodes have multiple antennas or the network has more than one cell. Particularly, SC-SIC is not optimal in multipleinput single-output (MISO) [245] and MIMO, and secure MIMO systems [224]. Despite the fact that the capacity of MIMO-BC is known [246], many NOMA researchers have applied SC-SIC to this channel [236,238], calling it MIMONOMA, which is sub-optimal. Recent works have tried to address this deficiency by focusing on optimal capacityachieving schemes for this channel. The optimal schemes are still non-orthogonal but not based on SC-SIC. Rate splitting and dirty paper coding (DPC) are among these schemes and are necessary for the spectral efficiency of MISO and MIMOBC channels [224, 245, 247].

\section{B. Random Access Solutions}

Packet flow through an LTE network needs coordination between the BS and the device. A device can be in an idle or active state [248]. When the device is idle, it is only listening to control channel broadcasts. Access protocols come into play when the device wakes up, i.e., its state changes from idle to active [248]. To wake up from an idle state, the device must synchronize with a nearby BS by sending a request for radio resources. For this purpose, an access protocol is used to coordinate the data exchange between multiple active devices and the BS [249]. Since the activity of the devices is random,

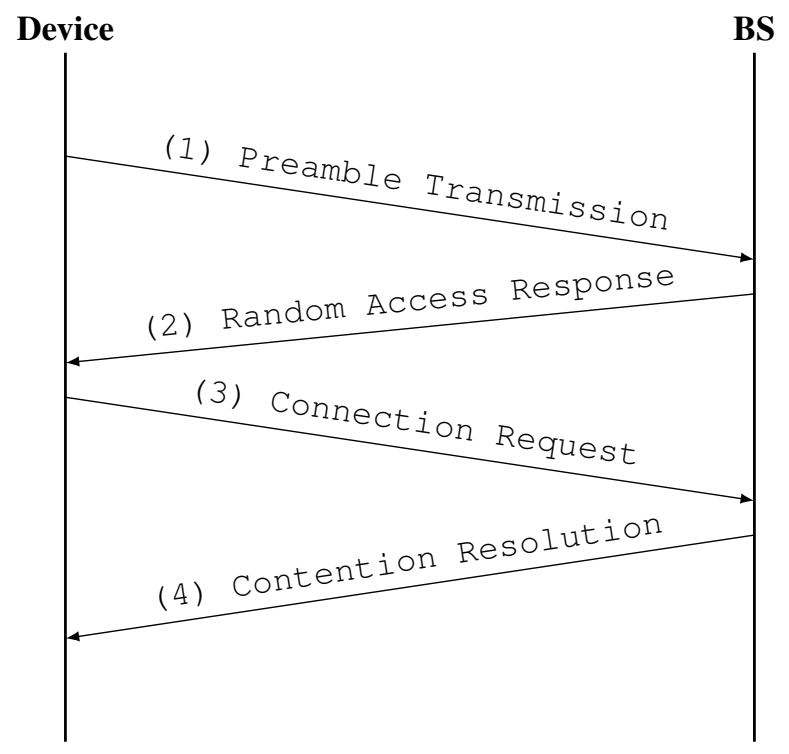

Fig. 15: A grant-based random access protocol.

random access protocols are used in cellular IoT [76]. Random access protocols can be divided into three types:

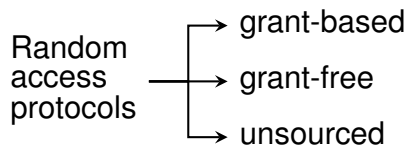

1) Grant-based random access: In a grant-based random access protocol, to access the network an active device needs to obtain permission from the BS before sending its payload data. This procedure has four steps, as shown in Fig. 15:

(1) Each active device randomly chooses a preamble out of a collection of orthogonal preambles and sends it to the BS to notify that the user has become active.

(2) The BS sends a response as a grant for transmitting in the next step.

(3) Each device receiving a response sends a connection request to ask for resource allocation for data transmission in the next step.

(4) If the preamble was chosen by 'only' one device, a connection resolution message is sent to that device informing which resource is reserved for it. Otherwise, the connection requests collide, and the connection resolution message is not sent to any of those colliding devices. In this case, those devices need to restart the procedure.

This procedure may take $10 \mathrm{~ms}$ or more [193], which is high above the 5G and beyond networks' latency requirement. Fur- 
ther, as the number of active devices goes up, the probability of collision increases and this time becomes higher. Today's $5 \mathrm{G}$ NB-IoT is a grant-based random access $[76,250]$

2) Grant-free random access: A grant-free transmission, on the other hand, eliminates the scheduling request for uplink data transmission. Unlike the grant-based case, the procedure starts with the BS detecting the active devices and sending them a unique preamble for all time slots. Next, the BS receives metadata and uses it to estimate the channel [250]. This can reduce the access latency compared to the grantbased access protocols. Device activity detection is the main challenge here as orthogonal preambles may not be available to all active devices due to their massive number and the limited channel coherence time.

In [250], the device-activity detection is cast into a compressed sensing problem, and an approximate message passing algorithm [251] is proposed to detect the active devices. This algorithm is used in several papers [251-253] that apply the massive MIMO technology to boost the deviceactivity detection for massive IoT connectivity. Recent works $[254,255]$ have used DL-aided methods to design preamble sequences and recover sparse signals effectively. In [256], Zadoff-Chu sequences are proposed for preamble design for their good auto- and cross-correlation properties. Several other approaches, such as Bayesian detection, joint device detection and channel estimation, joint device and data detection, are proposed in the literature [76]. With the exploding number of mMTC IoT, massive device detection in grant-free random access is an important problem in beyond 5G (B5G) wireless networks. Several of these challenges are discussed in Sec. IX.

Also, grant-free NOMA is extensively covered in [32].

3) Unsourced random access: Unsourced multiple access channel is referred to the multiple access problem introduced by Polyanskiy in [257]. This work sets an informationtheoretic basis for a typical mMTC scenario in which each of a massive number of uncoordinated users infrequently transmits a small payload (a b-bit message) to the BS. The BS is interested only in recovering the list of messages without being interested in the identity of the user who transmitted a particular message. Due to the small size of the message, the block length is also small. In [257], the number of users is comparable to the channel block length. Due to the unsourced, uncoordinated nature of the problem and the small block lengths, the problem is very different from the traditional multiple access channel and, consequently, has fundamental limits and coding schemes. The transmission is grant-free, but it is different from grant-based and grant-free random access protocols discussed earlier in that in unsourced massive random access each device does not get a unique preamble sequence, but all devices use the same channel codebook (sequences).

Since the introduction of this new information-theoretic setting several advances and extensions have been made. The first low-complexity coding for the unsourced multiple access channel was described in [258]. The extension to massive MIMO cases was introduced in [259]. The authors in [260] proposed chirp detection with higher computational efficiency and lower energy per bit. Despite these advances, many challenges remain to be addressed to bring unsourced massive random access into practice. More efficient codebook design and activity detection algorithms are two of them.

\section{IOT SECURITY}

Billions of devices are being connected to the IoT through many different communication protocols. Regardless of the underlying connectivity standard, be it the licensed cellular spectrum or the unlicensed spectrum, we can view these devices as networked systems that are vulnerable a) through physical means or b) remotely, through the network and through software exploits.

Most types of communication standards (NB-IoT/LTEM/4G/5G, LoRa, Sigfox) were not designed with end-to-end security in mind. Even though each of them provides some authentication and/or encryption solutions, they are not always applied correctly [261]. According to a recent IoT threat report, $98 \%$ of IoT traffic is unencrypted when $100 \%$ of the traffic should be encrypted [262]. The most obvious attack vector is a man-in-the-middle attack where attackers can get in between devices or in between the device and the larger enterprise network. Even though IoT devices might not be the actual target of an attacker, they provide the first point of entry in order to launch more serious attacks on other systems on the network.

Many IoT devices are intrinsically less secure than traditional personal computers or network servers. Limited by their size, memory and power constraints, these devices either do not have an operating system at all, or they can only run stripped-down versions of operating systems that do not have sophisticated security features. Intrusion detection algorithms and malware detectors are resource-intensive security tools that are normally not supported by IoT devices. 57\% of IoT devices are vulnerable to medium-or high-severity attacks, making IoT the low-hanging fruit for attackers [262].

As shown in Fig. 16, data from IoT devices (A) are typically transmitted wirelessly to a gateway/cellular base station/wireless access point (B). Gateways use a backhaul network to connect to a server/cloud (C) which processes the data transmitted by the IoT devices. After processing, information is transmitted using a transport layer security (TLS) protected connection (HTTPS protocol) to the client's application (D). In a 100\% secure IoT environment data have to be protected both at rest and during transmission through all intermediate hops. Even if the communication link from the IoT device (A) to the gateway (B) is protected, after that, modern IoT data still has a long way to go to its destination.

\section{A. IoT Security Attacks Based on Their Characteristics}

Numerous cyberattacks in the form of eavesdropping, impersonation, key extraction, jamming have impeded the successful deployment of cellular and wide-are IoT. Passwordrelated attacks continue to be prevalent on IoT devices due to weak manufacturer-set passwords and poor user practices. Recent reports on the IoT attack landscape indicate that attackers typically use botnets to conduct distributed denial-ofservice (DDoS) attacks or utilize malware that spread across 


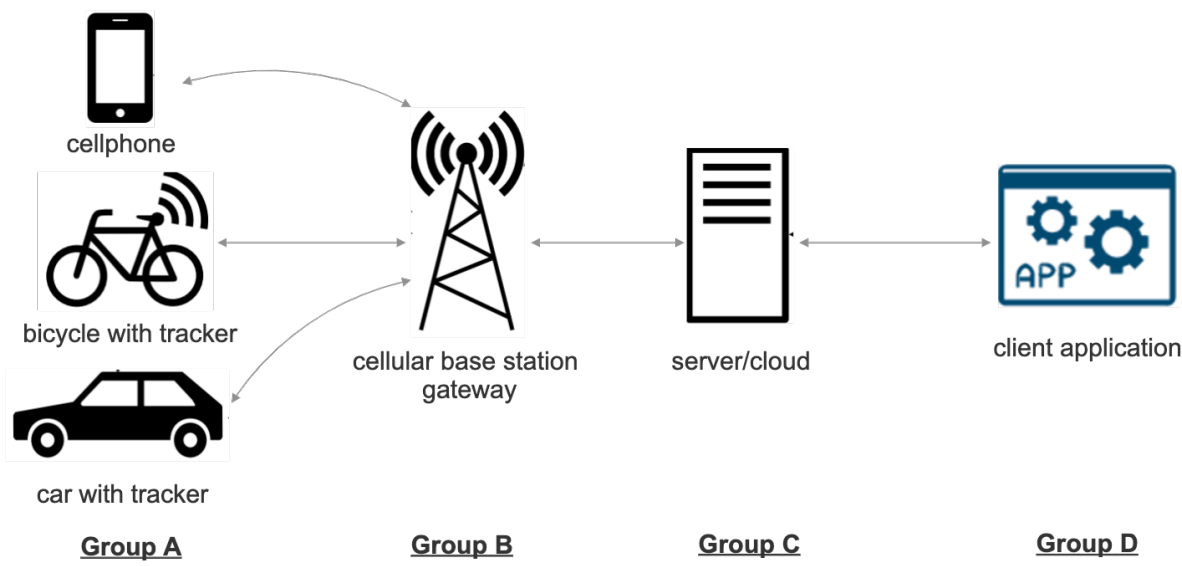

Fig. 16: Overview of devices and communication links in a typical IoT deployment.

the network [263]. Major topics such as key management and authentication continue to play a central role in current research [264].

In this section, we categorize the most prevalent attacks against cellular/wide-area IoT based on the characteristics of the medium used for each attack. Malicious entities a) either use physical means to compromise devices or intermediate gateways and serves, b) they attack the upper layer applications on the IoT device, or c) attack the network itself as shown in Fig. 17. The last type of attack is a major concern moving forward as the network of devices scales up; attacks can now propagate from one device to another and eventually have a more devastating effect on the IoT infrastructure.

1) Physical: Physical security is a necessary condition for the security of an IoT device. Any sophisticated cryptographic algorithm will no affect if the device can be simply disconnected or tampered with. Physical types of attacks either target the environment to produce malicious sensor readings [265] and cause false alarms, they target the hardware/software of the IoT device assuming that the attacker has physical access to the device, or they target the gateway that connects the IoT device to the rest of the network [266-269]. Such attacks include stealing cryptographic keys from the non-volatile memory of the device [270] and injecting false sensor signals that confuse the control logic of the system. The attacker could also target the gateway physically, in order to block the communication signals between the IoT device and the backhaul network. Jamming attacks are one of the most serious problems for IoT security since attackers can transmit radio signals near the devices with enough power that interrupts the radio communications. A low-cost micro-controller board like an Arduino board with a radio module can be used to perform jamming attacks. The main objective of the attacker is to transmit a wide-band signal with a higher SNR than the user [271]. Battery exhaustion attacks and disconnection attacks in LoRaWAN are other types of attacks with physical manifestation [272, 273].

2) Software: Software attacks on any device, not only on cellular/wide-area IoT, start from the operating system. If the attacker manages to bypass the operating system they can access parts of the filesystem that are private or they can execute untrusted code. Buffer overflows and structured query language (SQL) injections are some common exploits that lead to malicious code execution and breaches in privacy [274, 275]. It is worth noting that some IoT devices can not even support an operating system because of their size and memory constraints.

Under the same category, we have malware that can lead to accessing, copying, or altering of information, and backdoor trojans that can be inserted to constantly leak data [276]. Password based attacks account for approximately 15\% of IoT threats, poor user practices that disable strong passwords and the use of weak manufacturer-set passwords contribute greatly to the insecurity of IoT devices [262].

As we move to more decentralized network deployments (e.g. 5G) software is not only at the core of IoT devices, but at the core of the network infrastructure as well. At the same time, the security checks that earlier technologies were employed at a centralized level, are no longer possible. To add flexibility, networks have become programmable and virtualized in software. This increases the potential threats at the network level itself, since software testing is an undecidable problem [277] and there will always be loopholes that a determined attacker can exploit. Even though resources are considered isolated in sliced networks, there are concerns that isolated network partitions can still be accessed at the hardware level [278].

3) Network: Technologies like LoRaWAN, Sigfox and NBIoT have several authentication and data encryption schemes to protect data between the IoT device and the gateway [261]. However, recent publications reveal that wide-area IoT deployments are still susceptible to impersonation and availability attacks. One example is a replay attack, where a malicious entity captures and stores a duplicate of a genuine network packet that is used to authenticate the legitimate user, and then at a later point in time, the attacker "replays" this network packet to the system in order to get services that are only available to authenticated users [269]. To perform this attack in wireless networks, the malicious entity should know the communication frequencies and channels to sniff 


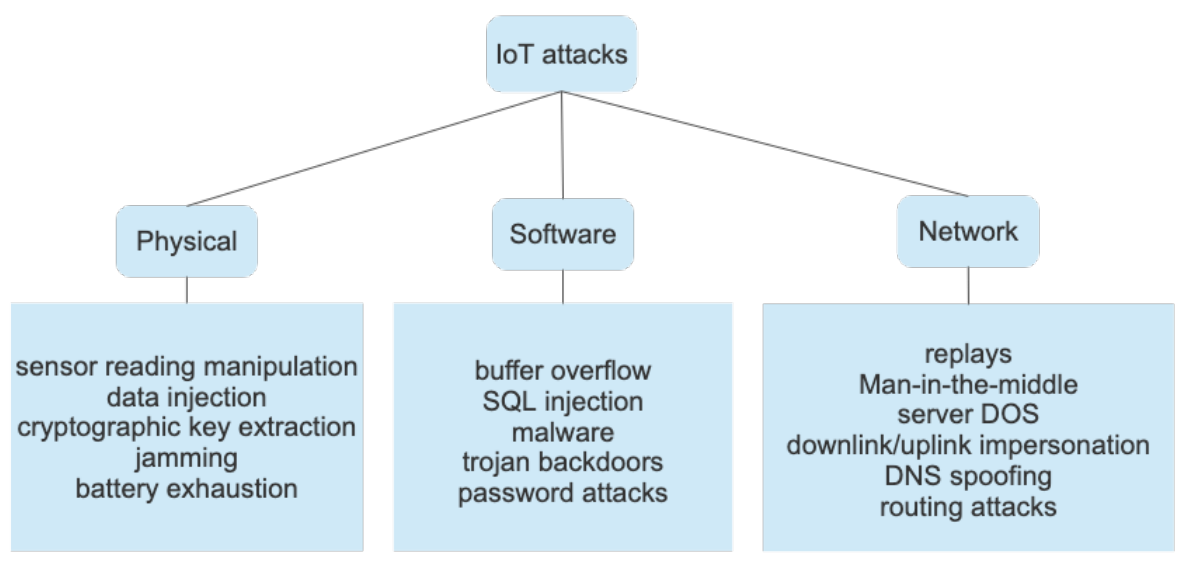

Fig. 17: IoT attack classification

data from transmission between devices [266, 267]. Routing attacks against low power wide-area networks are a wellstudied topic as well [279].

An attacker can also target the link between the gateway and the server $[268,272]$. Using a man-in-the-middle attack the malicious entity can take over the communication channel, and either delay/disrupt the message exchange, or modify the transmitted messages. Another target could be the wide-area network server itself. Multiple service requests can lead to a denial of service attack that overwhelms the server's resources and causes the server to malfunction.

$4 \mathrm{G}$ and $5 \mathrm{G}$ networks have made significant improvements to their security compared to former cellular technologies, like mutual authentication and key agreement (AKA) of the device and the cell tower. Control plane communication is kept confidential by applying encryption, but they are also protected against malicious modifications by using integrity protection schemes. Actual content information (data plane) is however only encrypted, not integrity protected. $5 \mathrm{G}$ networks enable integrity protection for content data - but this is only optional and not mandatory. At the same time, we have to consider the fact that non-standalone $5 \mathrm{G}$ deployments inherit all the security vulnerabilities of their predecessors since they are using older cellular technologies as their network core (LTE, $4 G)$.

An example of an attack that can affect 5G IoT was first published in [280]. The attacker takes advantage of the fact that there is no integrity protection on this channel, changes crucial parts of the encrypted transmitted information so that when those parts are decrypted the original message is no longer the same. Without knowing any encryption keys the attacker can perform an attack called domain name system (DNS) spoofing; i.e. changing the IP address of the DNS query issued by the target user equipment to the IP of a malicious DNS server. Of course, the malicious server is operated by the attacker, so the DNS request will be routed to the attacker. Such an attack could be very effective, overcoming the basic security capabilities of LTE and 5G, using the fact that no integrity protection was included.

Cellular denial-of-service (DoS) attacks have been one of the most popular attacks on cellular networks; attackers imper- sonate cell towers utilizing "authentication and key agreement fake towers" and convince devices to connect by providing the best signal. Once the device connects to the fake tower it is no longer available since it does not receive service.A recently published authentication attack was also reported in [281] and is based on a reflection attack where the response from a device includes a copy of a specific part of the request. The objective of the attacker is to modify the IP addresses of the victim IoT device on the uplink or the network server on the downlink. Now the attacker can get access to any service, assuming the victim's identity (uplink impersonation) or assuming the identity of any legitimate service provider (downlink impersonation).

\section{B. SDN and Blockchains in IoT}

One of the first studies that highlighted the importance of software defined networking (SDN) and blockchains in IoT is [282]. With billions of devices being connected to the same network, we need flexibility in resource allocation, network management, and a variety of new services that enhance the user's experience. At the same time, these emerging technologies can address the issue of key management, confidentiality, integrity, and availability in IoT systems [283285]. Blockchain and SDN can bring additional benefits to IoT security in terms of flexibility and scalability [286-289].

1) $S D N$ : SDN has emerged as a novel networking paradigm that revolutionized network deployments. It allowed for efficient network management, as well as the ability to launch new services with minimal effort. Programmability in software, offers great flexibility and scalability in the network infrastructure. In the pre-SDN world, the control and data plane were coupled in networking devices. With SDN, devices like switches, routers or IoT devices themselves do not have routing tables and access control lists; they merely forward packets, while intelligence has moved to the SDN controller. The controller can now run security services in a centralized manner and take all the routing decisions using protocols like Openflow. [290, 291] Devices in the SDN architecture handle packets based on flow tables setup at the SDN controller.

An SDN deployment can help resource-constrained IoT devices by optimizing the resource allocation problem. At 
the same time, many security issues can be overcome by moving security intelligence to IoT gateways. In [292], authors presented an SDN architecture for IoT devices based on Openflow, where IoT gateways, by analyzing network traffic, can identify abnormal system behavior and pinpoint which devices are misbehaving or which devices could potentially be compromised. Then, the gateway is entrusted with the task of taking appropriate action like a firewall would do, block the packet, forward the packet, and even classify it in terms of quality of service requirements.

Alternatively, the work in [293] moves security intelligence to some of the less resource-constrained IoT devices that are connected to the same network. The authors make the assumption that within the IoT infrastructure devices come with different capabilities, some have low power constraints but some have a robust operating system. The devices that are less resource-constrained in terms of computation and power consumption can assist by authenticating other IoT nodes and enforcing security rules using the Openflow protocol. In this way, secure, end-to-end connections can be established between IoT nodes, and man-in-the-middle attacks can be avoided.

2) IoT security, privacy and trust: Blockchain is a novel type of database that stores data in a unique way. It stores data as blocks that are chained together, such that any modification in intermediate blocks will result in an invalid chain. This concept can be applied in many modern application domains including cellular, wide-area, and non-terrestrial IoT deployments. The advantage of this new technology is that any transaction in the cyber-worlds can be validated in a distributed way using a consensus algorithm run by a set of users in the network called miners. Because of this property Blockchains can improve the security of IoT devices. If IoT devices store their data in a Blockchain misbehaving IoT devices will be singled out by the whole group.

As we can see in recent literature Blockchain-based IoT applications can take advantage of integrity and privacy by design, which is very important for the successful deployment of cellular and wide-area network IoTs at scale [286,294296]. Every piece of data that is part of a transaction is hashed as a block to protect its integrity. At the same time, public key cryptography is used, where each node in the IoT network gets its own set of public/private keys. They can use their private key to authenticate a transaction, since the only node who could have possibly made the transaction is the owner of the private key. They can also use another node's public key to encrypt a short transaction message and make sure that the only node that can decrypt it is the owner of the corresponding private key. As IoT continues to grow, security by design is the only way forward. IoT devices and particularly sensor-based IoT devices communicate location/temperature/vital signs; many data points per second are collected for data analytics and monitoring purposes. It is important to have an underlying infrastructure, like a Blockchain, that creates permanent records of those data sets. This, not only ensures the reliability of data but the overall security of the exchanged messages in terms all confidentiality, integrity, and authentication. Of course, public key cryptography is as good as its key exchange. Traditional ways of storing keys and exchanging certificates do not scale with the volume of IoT, so this is an area that future research needs to address.

\section{DEEP LEARNING FOR IOT}

Machine learning (ML) algorithms try to learn the mapping from an input to output from data rather than through explicit programming. ML uses algorithms that iteratively learn from data to describe data or predict outcomes, i.e., to produce precise models based on that data. As the training data becomes larger, it is then possible to produce more precise models. There are many learning algorithms and applications. Careful hand-crafted features are a critical component of the model building process in traditional ML algorithms. That is, a domain expert should extract and select the key features of the input before feeding them to the model. Feature engineering not only needs considerable expert knowledge but is also a very time-consuming process.

Three types of ML tasks can be considered: supervised learning, unsupervised learning, and reinforcement learning.

- Supervised learning is the task of inferring a classification or regression from labeled training data. ${ }^{6}$

- Unsupervised learning is the task of drawing inferences from unlabeled training data, i.e., input data without labeled responses.

- Reinforcement learning is the task of learning how to interact with an environment by taking a sequence of actions to maximize cumulative rewards.

\section{A. Neural Networks}

Artificial neural network (ANN), or simply neural networks, are learning algorithms (computing systems) that mimic the operations of a human brain to find the input-output mapping from large amounts of data. As shown in Fig. 18, the ANN consists of three layers: input layer, hidden layer, and output layer. Each layer consists of multiple neurons and tries to learn certain weights. The input layer accepts the inputs, the hidden layer processes the inputs, and the output layer produces the result.

A single neuron can be imagined as a regression classifier, which is a supervised ML algorithm. The neuron receives the inputs, multiplies them by their weights, adds them up, and then it applies the activation function to the sum. The activation function is used to normalize the output. It returns the output in the range $[0,1]$ which can be thought of as a probability. There are various activation functions. Linear activation function, sigmoid function, softmax, and rectified linear units (ReLU) [297] are examples of activation functions.

In supervised learning, a neuron learns the weights based on the inputs and the desired outputs through an optimization process in various phases. First forward propagation occurs where the input training data crosses the entire neural network and the output is predicted. Next, a loss function is used to estimate the loss (or error) between the correct output and

\footnotetext{
${ }^{6}$ Classification algorithms are used to predict/classify the discrete values such as an email is spam or not whereas regression algorithms are used to predict the continuous values such as price, salary, etc.
} 


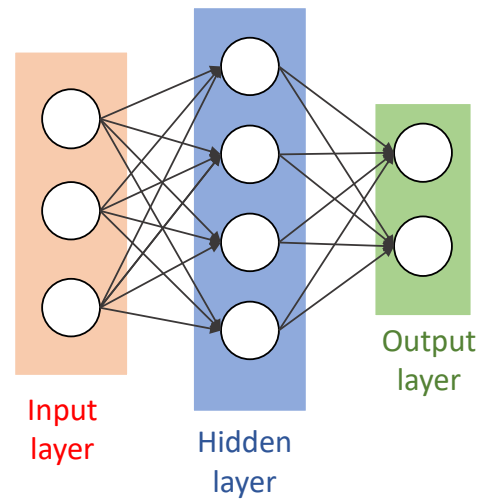

Fig. 18: An artificial neural network.

the predicted one. It measures how good/bad our prediction result is. The next phase is backward propagation in which the calculated loss is propagated backward to all neurons in the hidden layer, such that all the neurons in the network receive a loss signal that describes their relative contribution to the total loss. This information is then used to update the weights of the network through the gradient descent algorithm such that the next loss becomes smaller, and thus a better model is obtained. This process is iterated until we get a 'good' model, i.e., the loss approaches to zeros.

Due to their capacity to learn weights that map any input to the output, ANNs can learn any nonlinear function. This is the main advantage of ANNs which also makes them be known as universal function approximators. It should be highlighted that ANN ideas have been around for a long time. The main limitation of ANN is that it may over-fit easily, is slow to train, and is not great for images, time series, and large numbers of inputs-outputs. Today, ANN is a broad term that encompasses any form of DL model.

A basic three-layer ANN is comprised of

- Input layer: data is a vector, and each input neuron collects one feature/dimension of the input data and passes it on to the hidden layer.

- Hidden layer: each hidden neuron computes a weighted sum of all the neurons from the previous layer (input layer) and passes it through a nonlinear activation function.

- Output layer: Each output neuron computes a weighted sum of all the hidden neurons and passes it through a (possibly nonlinear) threshold function.

\section{B. Deep Learning Models}

Deep learning (DL) algorithms are a subset of ML algorithms. deep neural networks (DNNs) are ANNs with some level of complexity, usually, networks with two or more hidden layers. DNNs perform well in tasks like regression and classification. ${ }^{7}$ They can learn hidden features from raw data [298]. Each hidden layer trains on a set of features received from the previous layer and can recognize more complex

\footnotetext{
${ }^{7}$ The former deals with predicting a continuous value whereas the latter predicts the output from a set of finite classes.
}

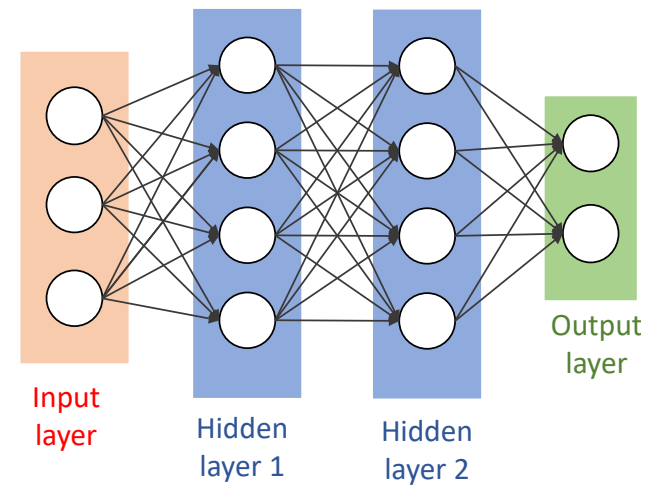

Fig. 19: A deep neural network.

features. So, a deeper network can result in more complex features and more accurate learning.

DNNs are changing the way we interact with the world. Before introducing the most common DNN models, we discuss what makes DL different from ML algorithms. There are two main reasons why researchers and experts tend to prefer DL over ML:

1) $\mathrm{DL}$ algorithms are capable of learning much complex decision boundaries. Hence, in general, DL models improve accuracy/performance.

2) DL automates the process of feature engineering or at least reduces the need for that. Recall that feature engineering is a key step in the model building process. It also needs expert knowledge and typically is very timeconsuming.

Therefore, compared to the conventional ML techniques, DL models improve the accuracy and reduce the need for hand-crafted features [298]. These make DL attractive to many people in different fields, not just machine leaning experts.

In addition to the above advantages which apply to almost any applications, embedding DL models to $5 \mathrm{G}$ and beyond mobile networks has several other justifications and advantages such as [299]

- There exist various DL models to learn useful patterns from unlabeled data, which is the most common type of data in today's mobile networks [300], in an unsupervised manner. Restricted Boltzmann machine (RBM) and generative adversarial network (GAN) are two examples of such DL methods.

- A single DL model can be trained to satisfy multiple objectives, without a need for complete model retraining for each objective [301,302]. This reduces computational and memory requirements of the systems which are particularly important in IoT networks.

- Mobile networks generate large volumes of heterogeneous data. DNNs not only can handle such data but also benefit from it since training with big data prevents model over-fitting. In contrast, traditional ML algorithms such as support vector machine (SVM) may require storing all the data in memory which makes scalability difficult [299]. 
TABLE VIII: Different types of deep neural networks and their characteristics

\begin{tabular}{|c|c|c|c|}
\hline Model & Description & Characteristics & Applications \\
\hline DNN & $\begin{array}{l}\text { Deep neural network is an ANN with } \\
\text { some level of complexity. Usually, a net- } \\
\text { work with more than two hidden layers. }\end{array}$ & $\begin{array}{l}\text { Dense connectivity in each hidden layer } \\
\text { - Matrix multiplication in each layer }\end{array}$ & \\
\hline CNN & $\begin{array}{l}\text { A convolutional neural network (CNN) } \\
\text { applies multiple } 2 \mathrm{D} \text { filters to the input to } \\
\text { extract features (convolution operations). } \\
\text { A CNN is a DNN that uses convolution } \\
\text { instead of general matrix multiplication at } \\
\text { least in one of its layers. Convolution has } \\
\text { sparse connectivity. }\end{array}$ & $\begin{array}{l}\text { - Convolution layers for feature extraction } \\
\text { - Less connection compared to DNNs } \\
\text { - Simple (sparse connectivity) and efficient } \\
\text { for image } \\
\text { - Takes a long time to train }\end{array}$ & $\begin{array}{l}\text { - Image recognition } \\
\text { - Video analysis } \\
\text { - Document analysis }\end{array}$ \\
\hline RNN & $\begin{array}{l}\text { Recurrent neural networks (RNNs) are } \\
\text { the time series version of ANNs and } \\
\text { are meant to process sequences of data. } \\
\text { LSTM (long short term memory) is the } \\
\text { most common type of recurrent layer in } \\
\text { which past information can flow through } \\
\text { the model. }\end{array}$ & $\begin{array}{l}\text { - Links outputs back to inputs over time } \\
\text { - Good for time series learning } \\
\text { - Models and uses "memory" } \\
\text { - Useful for extracting information from } \\
\text { time-dependent and time-correlated data } \\
\text { - Understands the temporal nature of data }\end{array}$ & $\begin{array}{l}\text { - Robot control } \\
\text { - Speech recognition/synthesis } \\
\text { - Machine translation } \\
\text { - Mobility detection } \\
\text { - Predict next value in a sequence }\end{array}$ \\
\hline GAN & $\begin{array}{l}\text { A generative adversarial network is com- } \\
\text { posed of two networks: generator and } \\
\text { discriminator networks. The former gen- } \\
\text { erates new data instances, whereas the } \\
\text { latter evaluates them for authenticity. }\end{array}$ & $\begin{array}{l}\text { - Train two networks at once } \\
\text { - Similar to deep reinforcement learn- } \\
\text { ing (DRL) but does not require a } \\
\text { value/reward function } \\
\text { - Can be used together with CNN, RNN, } \\
\text { etc. }\end{array}$ & $\begin{array}{l}\text { - Generating photographs } \\
\text { - Text-to-image translation } \\
\text { - Image editing/inpainting } \\
\text { - Improving cybersecurity } \\
\text { - Tumor detection }\end{array}$ \\
\hline $\mathrm{AE}$ & $\begin{array}{l}\text { An autoencoder (AE) is an ANN that } \\
\text { learns to copy its input to its output. It } \\
\text { tries to reconstruct the original input by } \\
\text { minimizing the reconstruction error (the } \\
\text { differences between the input and recon- } \\
\text { structed version of that) }\end{array}$ & $\begin{array}{l}\text { - AE is an unsupervised learning technique } \\
\text { - AEs are data-specific } \\
\text { - Same input and output size }\end{array}$ & $\begin{array}{l}\text { - Anomaly detection } \\
\text { - Information retrieval } \\
\text { - Data denoising } \\
\text { - image Inpainting } \\
\text { - E2E communication [303] }\end{array}$ \\
\hline
\end{tabular}

Besides their various advantages for mobile and IoT networks, there are also several limitations for the application of DL in these domains. In particular, DL models are vulnerable to adversarial examples, they heavily rely on data but data collection may be costly or infeasible in some cases due to the privacy constraints, they can be computationally expensive for many IoT device, and they have many hyperparameters and finding their optimal configuration is not trivial, and DL algorithms have low interpretability [299].

Table VIII describes several types of NN that are used to solve DL problems and summarizes their salient characteristics. In the following we describe several of these DL models as well as their application related to IoT networks. Nonetheless, several important questions need to be answered if IoT devices and mobile apps can effectively implement DNN technology [304-306]:

- What DNN structures can effectively process and fuse sensory input data for diverse IoT applications?

- How can DL models be efficiently deployed on resourcelimited IoT devices? In other words, how can we reduce the resource consumption of DNNs to make them suitable for IoT devices?

- Deploying DNNs on resource-constrained IoT devices is a big research challenge. If such DNNs are implemented on the cloud where powerful resources are available what new challenges will arise?

- How can we minimize the need for labeled data in IoT learning?

- What distributed learning methods are relevant to the IoT realm?

- What is the state-of-the-art and the outlook for DNN accelerators that can operate in diverse IoT applications?

As an example, DNNs can be used successfully in mobile apps provided that they can collect data from various IoT devices and analyze them energy-efficiently and accurately using minimal data labels. While research in this field is very vibrant, there are already promising solutions out. We will present some of those solutions in this section.

\section{Autoencoder}

An AE is a neural network that is trained to learn efficient representations of the input data, that is, it learns to copy its input to its output. AEs are mainly used for solving unsupervised problems. These networks first compress (encodes) the input and then reconstruct (decodes) the output from this representation by minimizing the differences between the input and reconstructed version of the input. The point behind data 
compression is to get a smaller representation of the input and passed it around. This compressed representation should keep data quality such that people can recreate it when needed. ${ }^{8}$ Since AEs are expected to create a copy of the input, they are great for fault detention, intrusion detection, disease diagnosis, and in general for anomaly detection as described below. are

- Anomaly detection: Anomaly detection (or outlier detection) refers to the process of identifying rare items or events in data sets that do not conform to expected behavior i.e., differ significantly from the norm [310]. Anomaly detection is based on two assumptions: (i) anomalies occur very rarely in the data, (ii) they have significantly different features compared to the normal instances.

Anomaly detection is important because in many applications anomalies in data correspond to critical and actionable information. For example, an anomalous traffic pattern in a computer or an IoT device could mean that the device is compromised or hacked. Applications of anomaly detection are wide and increasing. Examples include but are not limited to fraud detection for credit cards, intrusion detection for cyber-security, fault detection in industry, and medical diagnosis in healthcare. DL techniques, including AE-based ones, have been proposed to address security threats such as anomaly and intrusion detection in IoT networks [311]. Some recent AE-related works can be found in Table IX.

- Intrusion detection: An intrusion detection system (IDS) is a device software that monitors network traffic for suspicious activities and reports such a harmful activity or policy breaching to an administrator. Suspicious activities may also be collected centrally using a security information and event management system to differentiate malicious activity from false alarms [312]. IDS prevents IoT botnet propagation as well as outbound attack traffic. There are various IDS systems such as network IDS, host IDS, protocol-based IDS, and hybrid. Yet, there are two main detection methods: signature-based detection and anomaly-based detection. The former detects attacks based on specific patterns (such as the number of bytes in the network traffic) or the already known malicious instruction sequence that is used by the malware. The latter, however, detect unknown malware attacks. It classifies system activity as either normal or anomalous and this classification is based on heuristics or rules, rather than patterns or signatures.

As new malwares are developed rapidly, anomaly-based detection has become more and more important. Further, the fact that ML and DL can be used for the classification of activities [313], has given momentum to this type of detection. ML/DL can be used to create an activity model to declared an activity is suspicious or not. Such models can be trained based on the applications and hardware and thus can perform better than the signature-based

\footnotetext{
${ }^{8}$ The idea is similar to distributed source coding techniques [307-309] where a compressed version of a signal is transmitted to a receiver that is expected to reconstruct the original data from that. Hence, such techniques may be useful in this context.
}

IDS in practice. Various deep AE-base IDSs have been introduced in recent years. We list several of them in Table IX.

\section{Deep Reinforcement Learning}

Reinforcement learning (RL) is a type of ML where the system learns how to behave in an environment by performing actions and seeing the results. More precisely, an agent learns from an uncertain environment by interacting with it and receiving rewards for performing actions, as we do in our natural experiences. In other words, trial and error is employed to train ML models that make a sequence of decisions to attain a complex objective (goal) or how to maximize along a particular dimension. ${ }^{9}$ For instance, the goal could be maximizing the points won in a game over many moves. We want the RL agent to earn lots of reward. The agent must exploit actions already knows to obtain a reward. It must also explore/select untested actions to discover new reward-producing actions. Thus, the action selection is a trade-off between exploration and exploitation.

DRL combines ANN with a reinforcement learning architecture that enables agents to learn the best actions to attain their goals. It has successfully trained computer programs to play games like AlphaGo and beat the world's best human players. DRL has found applications in many fields like robotics, computer vision, natural language processing, energy, and transportation [325]. Compared to RL, the DRL is much better for practical uses in many applications. For example, to achieve acceptable training times for practical uses in robotics, RL typically needs hand-crafted policy representations which compromises the autonomy of the learning process. DRL, in contrast, relieves this limitation by training general-purpose neural network policies [326].

Applications of DRL in IoT are diverse and numerous. We list several representative works in Table $\mathrm{X}$, and elaborate on some of them in the following. In general, DRL can be used in (i) perception layer (physical autonomous system), (ii) physical and network layer of IoT communication networks, as well as (iii) application layer (IoT edge/cloud computing) [33]. Robotic manipulation, autonomous driving, and energy storage management in smart grids are examples of the first one. Gu et. al [326] have used DRL to learn complex robotic manipulation skills on real physical robots.

In [327], a DRL-based scheduler is proposed to dynamically adapt to traffic variation, and to reward functions set by network operators, to schedule IoT traffic to avoid congestion. This work is motivated by IoT applications that need a high volume of data but have flexible time and thus their traffic is not needed to be scheduled immediately when it originates. In [333], a deep Q-learning solution for handover and radio resource management in cellular networks hosting aerial devices, drones, along with terrestrial users has been proposed. In [329] a framework of performance optimization for blockchain-enabled IIoT systems is proposed that aims to improve the scalability of the underlying blockchain without

${ }^{9} \mathrm{RL}$ is typically modeled as a Markov decision process (MDP). 
TABLE IX: Applications of deep AE in IoT

\begin{tabular}{|l|l|l|l|}
\hline Ref. & Model & Application & Contribution \\
\hline \hline $\begin{array}{l}{[314],} \\
{[315],} \\
{[316]}\end{array}$ & AE & Industrial IoT & Anomaly detection for industrial robots \\
\hline$[317]$ & RNN + AE & Sensor networks & Anomaly detection for IoT time series \\
\hline$[318]$ & AE & Smart logistics & $\begin{array}{l}\text { Anomaly detection as a service in cellular IoT for both IoT devices } \\
\text { and core network }\end{array}$ \\
\hline$[319]$ & DRL + AE & Smart city & Indoor localization based on Bluetooth low energy signals \\
\hline$[319]$ & AE & Healthcare & Heart diseases diagnosis \\
\hline$[320]$ & AE & Generic & $\begin{array}{l}\text { Intrusion detection using AEs to learn normal behaviors of the IoT } \\
\text { devices }\end{array}$ \\
\hline$[321]$ & AE & WiFi-connected IoT & $\begin{array}{l}\text { Attack detection using deep AE and feature abstraction based on WiFi } \\
\text { networks database [322] }\end{array}$ \\
\hline$[323]$ & AE + CNN & IoT gateways & Intrusion detection by examining as few packets as possible \\
\hline$[324]$ & AE + SVM & Generic & Intrusion detection \\
\hline
\end{tabular}

TABLE X: Some representative works on DRL in IoT

\begin{tabular}{|c|c|c|c|}
\hline Ref. & Model & Application & Contribution \\
\hline [327] & DRL & Broadband IoT & $\begin{array}{l}\text { A scheduler that can dynamically adapt to traffic variation to schedule } \\
\text { IoT traffic }\end{array}$ \\
\hline [328] & DRL & Mobile crowdsensing & $\begin{array}{l}\text { A framework for mobile crowdsensing in fog environments with a } \\
\text { hierarchical scheduling }\end{array}$ \\
\hline [329] & DRL & IIoT & $\begin{array}{l}\text { Blockchain-enabled IIoT systems to improve the scalability of the } \\
\text { blockchain without sacrificing latency, and security }\end{array}$ \\
\hline [319] & $\mathrm{DRL}+\mathrm{AE}$ & Smart city & Indoor localization based on Bluetooth low energy signals \\
\hline [326] & DRL & Robotics & $\begin{array}{l}\text { Asynchronous DRL for robotic manipulation trained on real physical } \\
\text { robots }\end{array}$ \\
\hline [242] & DRL & IoT massive access & $\begin{array}{l}\text { DRL implemented in a real BS for user pairing and resource alloca- } \\
\text { tion in cache-aided non-orthogonal multiple access for IoT }\end{array}$ \\
\hline [330] & DRL & $\begin{array}{l}\text { Resource } \\
\text { management }\end{array}$ & $\begin{array}{l}\text { The resource and task management states are transferred into images } \\
\text { as the input of the DNN. }\end{array}$ \\
\hline [331] & DRL & Edge computing & $\begin{array}{l}\text { DRL algorithm is proposed for the computation offloading in the } \\
\text { heterogeneous edge computing server. }\end{array}$ \\
\hline [332] & DRL & Smart agriculture & $\begin{array}{l}\text { DRL is applied in the cloud layer of a smart farming system to make } \\
\text { smart decisions, like irrigation, for improving crop growth. }\end{array}$ \\
\hline
\end{tabular}

affecting the system's decentralization, latency, and security. Doan et al. [242] applied DRL for resource allocation in cache-aided NOMA for IoT and showed that DRL increases the probability of successfully decoding at the users. In [319] DRL is used for indoor localization based on BLE signals. These are just a few examples of DRL applied to improve IoT communications in the physical or network layers.

Finally, DRL has also been used in the application layer for data processing and storage. An application layer agent may locate in the IoT edge/fog/cloud computing systems. Task offloading, resource allocation, and caching are examples of tasks in this category. Applying DRL for resource management was first proposed in [330], where the authors transferred the resource and task management states into images as the input of the DNN. The work in [242] applied DRL for resource allocation in cache-aided NOMA is an example. Li et al.
[328] have proposed DRL for mobile crowdsensing in fog environments. DRL provides a scalable, elastic, and selfadapting method to adapt to varying fog computing structures and mobile environments. In [331] a DRL algorithm is proposed for the computation offloading in the heterogeneous edge computing server. The IoT device performs computation offloading for each task request according to the decision given by a DRL model. Lastly, in [332] a smart agriculture IoT system based on DRL is proposed. DRL is applied in the cloud layer to make smart decisions like controlling the amount of water needed to be irrigated for improving crop growth environment.

\section{E. Distributed and Federated Learning for IoT}

ML algorithms build mathematical models from training data. Such models can be built in different ways: 


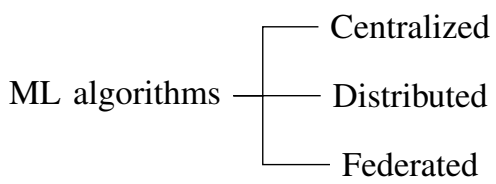

Standard ML algorithms collect data and train and validate the models in a centralized cloud server. In centralized ML all of the training and validation data resides in one location where the model is validated, verified, and frozen before its deployment. Centralized ML is not appropriate when training data is not in one location and there are sharing constraints. In such cases distributed ML or Federated learning is preferred. In distributed ML the learning/inferencing processes can be distributed depending on the needs of the system to overcome computational/storage constraints. That is, the training data is moved for processing by multiple computers. Distributed ML often uses parallel processing techniques. Federated ML is decentralized learning in which, unlike distributed learning, training data is not exchanged among the servers. This method is appropriate when there are concerns or constraints for sharing training data, e.g., due to policy, security, insufficient network capacity (wireless networks). Distributed and federated learning approached have great potential for IoT networks as we discuss in the following.

1) Distributed learning for IoT: Reducing signaling between IoT devices and the access network, e.g., over the unlicensed spectrum, results in decreasing energy consumption per data transfer for IoT devices at the cost of decreasing control over radio resource usage for the access network. For example, in the case of IoT solutions over the ISM band, there is almost no centralized control over the devices' transmissions. This results in no performance guarantee for IoT communications in this band $[103,334]$. In order to benefit from the relaxed transmissions, and suffer as low as possible from the uncoordinated access, there is a need for distributed solutions able to adapt communications parameters of devices to the environment. In recent years, there is an ever-increasing interest in leveraging machine learning tools in operation control of independent entities which have limited observability on their environments [335]. In [335], distributed reinforcement learning at the devices has been proposed for overload control in serving massive IoT traffic over LTE networks. Self-organized clustering and clustered access for IoT have been proposed in [336]. In [337,338], the multiarm bandit has been proposed for distributed management of IoT parameters, in which, devices learn how to avoid subchannels suffering from a high level of interference. The presented approach in [337] leverages two notions of external and internal regrets, for targeting energy consumption and collision probability, respectively, and is able to converge to the centralized optimized approach, e.g. [339], if network state (interference statistics) is constant for a longer period of time.

While regarding the limited computation/storage of IoT devices, deploying deep and complex AI algorithms seems impossible on most IoT devices, this area of research is expected to attract more attention in future works by adapting AI algorithms to the device constraints or splitting learning between the device and the network.
2) Federated learning for IoT: federated learning (FL) is a new ML framework that is distributed over mobile devices. The core concept of FL is to send the algorithm to the data rather than sending data from user devices to the server. With this, there is no direct access to labeling of raw user data, and thus, the model development, training, and evaluation in FL are different from centralized ML. FL was coined by Google [340] and has remained at the forefront of the field. In FL, both the central server and edge devices contribute to the model training process collaboratively via the following steps:

1) The central server chooses a model to be trained.

2) It sends the initial model to thousands of edge devices.

3) The devices train the model locally with their own data.

4) The central server gathers all the model results and generates a global model without accessing any data.

5) This process is repeated over and over again.

Once the training is completed, test versions of the algorithm are sent to many devices to evaluate the performance on real data. This step can also be iterated until an updated model is sent to all devices to replace the old ones.

Privacy has been the main motivation for devolving the FL. Due to its distributed model training, FL provides highly personalized models and does not compromise user privacy. FL has already been deployed in Google keyboard (Gboard) to improve word suggestions to the user without compromising their privacy. FL however offers several other advantages. Security and speed are other reasons that FL can work better in certain use-cases. Several advantages and limitations of FL are listed in Table XI.

When it comes to IoT applications, one main limitation of FL is that it requires high local device power and memory to train the model. For this reason, FL cannot be directly applied to sensors and resource-limited devices. However, with the rapid increase in the computational and storage power of smart devices, there are several potential scenarios for adopting FL in IoT applications in the future:

- Self-driving cars: it is expected to have are a large number of self-driving cars on our roads in the future. Since FL can reduce latency, a likely future scenario is to use FL in those vehicles to be able to rapidly respond to each other during safety incidents.

- UAVs: similar to driver-less cars, UAVs are increasingly used in various applications such as for delivery services, emergency response, disaster relief, agriculture, waste management, telecommunication, and Internet access. Another likely scenario for using FL is in UAVs to enable them to rapidly respond to each other during safety incidents since FL can reduce latency.

- Edge AI: Owing to the growth of smartphones and other smart devices, modern networks generate a huge amount of distributed data every day. Personalized features that are invaluable for many apps can be achieved on device.

- Mobile edge computing (MEC): While cloud computing and cloud RAN [341,342] offer unquestionable economies of scale, MEC enjoys compute and storage resources close to the end users, typically within or at the boundary of operator networks. Benefits edge solutions 
include low latency, high bandwidth, device processing and trusted computing and storage. Deep integration of computing in the network is expected to blur the line between the device, the network edge, and the cloud in the future, and as a result, distributed algorithms and applications will flourish.

- Robotics and smart industry: Robotic is a key component of Industry 4.0. Robots will operate in increasingly complex environments in a cooperative manner. Google is providing infrastructure essential to building and running robotics solutions for business automation. The literature on FL for robotics is sparse. Liu et al. [343] have investigated an FL scheme for cloud robotics. The proposed solution enables the cloud to fuse heterogeneous knowledge from local robots to develop guide models for robots. It makes navigation-learning robots use prior knowledge and adapt to a new environment. In [344] a secure FL-based data collaboration framework is proposed and validated the effectiveness of the proposed framework in a real IoT scenario.

Collaborative FL is a new approach for resource-constrained IoT. The concept is similar to D2D communication. IoT devices that are not able to communicate with a BS due to their limited resources send their local learning models to nearby devices rather than the BS. Some recent overview papers on federated learning for IoT networks can be found in [345347].

\section{F. IoT-Friendly DL}

State-of-the-art deep learning models usually involve millions to billions of parameters and need sophisticated computing and large storage resources to evaluate and save such a huge number of parameters. As an example, the number of parameters in commercial CNNs in computer vision applications is as high as $\sim 10^{8}$ [348]. Such huge models are computationally expensive for resource-constrained IoT and edge devices (e.g., smart sensors, wearables, smartphones, robots, drones, etc.), which have limited processing power, battery energy, and memory. For this reason, many DL methods can hardly be used in such devices for training purposes. Even worse, resources may not be sufficient for the test phase where a pre-trained model is run on the device [349]. Even if the devices have enough memory and computation resources, it is of great value to reduce the need for such resources to save energy, cost, and time. Therefore, there are growing interests in developing efficient DNNs.

Several other research teams have recently shown that the complexity of DNN models can be largely reduced without a significant drop in the accuracy. In the following, we discuss techniques used to achieve such results and illustrate some important results for each technique. The effort in this field can be categorized into network compression and acceleration techniques. We elaborate on these in the following.

1) Network compression: With negligible degradation of performance, DNNs can be compressed in various ways including low-rank tensor approximations, weight pruning, quantization, building a compact model, and a combination of two or more of these techniques. We describe these methods in the following.

i. Low-rank tensor approximations: Let us consider CNN as an example. It is well-understood that the bulk of computations is from convolution operations wheres most weights of CNNs are in the fully connected layers [348]. The fully-connected layer can be view as a 2D matrix (or $3 \mathrm{D}$ tensor). Approximating the matrices corresponding to those layers is a one compression method. The approximation can be performed in different ways including matrix decomposition. In this method, singular value decomposition (SVD) of those matrices is found and only singular values with large magnitude are kept. This lowrank approximation will be useful if the singular values of the matrix decay rapidly, and it could decrease the computation complexity up to an order of magnitude [348]. Other matrix decomposition methods like QR decomposition and CUR decomposition are proposed in the literature but are less accurate compared to the SVD method [306]. It is worth mentioning that lowrank decomposition can be also used for reducing the convolution layer. The convolution wights can be viewed as a 4D tensor and low-rank up the evaluation of CNNs [350]. There are also other side benefits for SVD. It can reduce memory footprint and decrease the number of learnable parameters which may improve generalization ability.

ii. Pruning: Consider an initially trained $\mathrm{NN}$ with a reasonable solution. One can remove all connections whose weight is less than a threshold and re-train the network until a reasonable solution is obtained [351]. Such a method permanently removes less important links and converts a dense, fully-connected layer to a sparse layer. Pruning process can be iterated to increase the compression efficiency. One may also use pruning with rehabilitation to improve learning efficiency [352]. In this method, there is no chance for the pruned connections to come back and incorrect pruning may cause severe accuracy loss. The above pruning methods are magnitude-based. There are other methods like the Hessian-based method. The idea is to delete parameters with small saliency, i.e., small effect on the training error [353].

iii. Quantization: This method compresses the original network by reducing the number of bits required to represent each weight. Unlike pruning which permanently drops less important connections and thus reduces the number of weights, this approach compresses the weights. In addition to weights, other data objects such as activation, error, and gradient can be quantized. Quantization is proved to be an effective way for model compression and acceleration [354-356]. In the extreme case of the 1-bit representation of each weight [357] binary neural networks are realized. The accuracy of binary nets is not impressive and needs further improvement [358]. Pruning, quantization, and other compression methods, e.g., source coding techniques, can be combined to increase the degree to which a network can be compressed. Han 
TABLE XI: Federated learning compared to centralized learning

\begin{tabular}{ll}
\hline Advantages & Limitations \\
\hline - Enhances user privacy by keeping personal data & $\begin{array}{l}\text { - Requires significantly more local device power } \\
\text { and memory to train the model }\end{array}$ \\
$\begin{array}{l}\text { on a person's device } \\
\text { - Lower latency as the updated model can be used }\end{array}$ & $\begin{array}{l}\text { - Requires higher bandwidth over WiFi or cellu- } \\
\text { lar networks during training }\end{array}$ \\
- Less power consumption at the central server as & - Insufficient bandwidth could increase latency \\
models are trained on user devices & and makes the learning process slower \\
- Less data transfer and thus faster learning pace & - Devices may drop out during the training pro- \\
- Deliver both global and personalized models & cess by their owners \\
- Accelerating clinical research as diseases dataset & \\
can be unlocked with less privacy concerns &
\end{tabular}

et al. [359] proposed a deep compression technique that can reduce the size and reduce the storage requirement of DNNs more than an order of magnitude without losing accuracy. This approach rains the initial network until a reasonable solution is obtained. It then prunes the network with a magnitude-based method until a reasonable solution is obtained. Next, it quantizes the network with k-means based method until a reasonable solution is obtained. Finally, it compresses the weight using Huffman coding.

iv. Compact model: Unlike the previous models that start with a dense model and then try to compress it, the idea behind a compact model is to design small CNN architecture from scratch while preserving acceptable accuracy $[360,361]$. Various techniques are introduced for this purpose. For instance, SqueezeNet replaces $3 \times 3$ filters of AlexNet with 1x1 filters, which creates a compact model reducing filter parameters 9 times. It also reduces the number of input channels to $3 \times 3$ filters resulting in about 50 times overall parameters reduction. Similarly, MobileNetV2 [360] is a family of general-purpose computer vision DNN designed with mobile devices in mind to support classification, detection, and more.

2) Hardware acceleration: Designing specialized hardware for efficient processing of DNNs is another active line of research that aims to minimize energy and storage required for DL models in general, and in IoT devices in specific. Such hardware is named neural network accelerators. Conventional DNN accelerators were only based on the hardware architecture to improve computation and reduce memory requirements. Parallel computing and processing in memory are two main techniques to this end [306]. However, with the exhaustion of parallelism and data reuse, sole hardware optimization reaches its performance upper bound. To overcome this, recent works have investigated algorithm and hardware co-design. Algorithm-hardware codesign has great potential in reducing the hardware cost. For this reason, modern accelerators have been exploited several of the network compression techniques discussed earlier.

Combining AI and edge devices with rich sensors, have great real-world applications in smart homes, autonomous driving, drones, and so on, and can dramatically expand the scope of AI applications. DNN on IoT devices based on microcontroller units is, however, extremely challenging. The memory (static random-access memory (SRAM)) of microcontrollers is about 3 orders of magnitude smaller than that of mobile phones and 5-6 orders of magnitude smaller than cloud GPUs [362], making it impossible to run off-the-shelf DNN models, even the compact models like MobileNetV2 [360].

Efficient DL computing and model compression is now an active line of research. A comprehensive survey of model compression and hardware acceleration for neural networks can be found in [306].

\section{NTN INTEGRATION INTO IOT NETWORKS}

A truly global and 3D connectivity of IoT cannot be realized by current cellular and wide-area networks. For example, in rural areas such as mountains, forests, and oceans, terrestrial infrastructure may not exist to enable such connectivity. For these cases, satellites networks play an important role in providing global connectivity. In addition, satellites are of crucial assistance when terrestrial networks are overloaded or out of service for instance in disaster scenarios. The terrestrial cellular coverage can be extended and complemented by the inclusion of satellite networks. On the other hand, the more favorable line-of-sight (LOS) propagation condition with satellites allows the IoT devices to lower down their transmit power and hence consume less energy. Therefore, satellite integration into IoT networks may provide significant advantages. Motivated by these facts, recent 3GPP study items include providing NB-IoT via satellite systems [363,364].

Furthermore, increased use of UAVs in novel commercial applications is currently being enabled by recent advances in the design of cost-effective drone technology $[365,366]$. A swift and infrastructure-independent deployment of UAVs particularly can be beneficial for disaster and poorly covered remote areas. As compared to satellite systems, UAVs deployments are more flexible, less expensive, and closer to the ground IoT nodes. The communication latency with UAVs is lower and the throughput can be higher at the expense of a 
shorter footprint and lifetime [367]. That is, UAVs complement satellite systems in supporting IoT networks.

In short, UAV-based and satellite-based networks provide global, 3D, and continuous coverage and are generalizations of cellular and wide-area networks. In this article, the term NTN refers to both UAV and satellite systems. In the following, we classify the main roles of NTNs integrated into IoT networks and elaborate on their particular advantages and challenges ahead.

\section{A. UAVs Integration into IoT Networks}

Three fundamental roles of UAV-aided IoT networks are as follows:

1) IoT data collection with UAVs: UAVs can be deployed to collect data produced by the low-power IoT devices or wireless sensors distributed over a certain region such as in precision agriculture applications. Such data might be delaytolerant information and hence does not need an instant connection to the central unit using permanent expensive ground infrastructure. Nevertheless, a real-time communication can also be facilitated by using UAV relays between IoT nodes and BSs. In effect, UAVs can fly above the ground nodes and establish LOS connection with both the ground nodes and the target BSs. Therefore, UAVs are capable of addressing several challenges in IoT-centric scenarios. Specifically, the communication links turn to be more reliable, the coverage range is potentially extended, and the energy consumption of the IoT devices can be reduced. In [368], a UAV-enabled IoT system is considered where a UAV collects data from IoT devices. The maximum energy consumption among all the devices' is minimized by jointly optimizing the devices transmit power, communication scheduling, and the UAV trajectory. In [369], the authors design the trajectory of UAV for data collection using the RL approach, in order to prolong the UAV flight time. To minimize the UAV data collection time, several other network parameters, including UAV height, velocity, and link scheduling, in addition to the UAV trajectory, are optimized in [370]. To improve the reliability between ground IoT nodes and BSs, authors in [371,372] study the performance of UAVs relaying collected data to BSs and propose efficient transmission algorithms.

In [373], an efficient path for maximizing the collected data under flying time and obstacle avoidance constraints is designed using a double deep Q-network approach. To maximize the energy efficiency of the IoT network, a deep RL approach is adopted in [374] through joint optimization of the power and channel allocation to the IoT devices in the uplink.

2) Localization of IoT devices with UAVs: In various applications of wireless networks location-aware services are required. In effect, the location information enables a more meaningful process of the gathered data and allows to optimize the network performance accordingly. As for the outdoor use cases, one may consider the global positioning system (GPS) where most of the time it provides satisfactory performance. However, such technology particularly for IoT devices is expensive and power-consuming. Moreover, GPS is vulnerable to jamming which might be a crucial drawback for a reliable and secure provision of services [375]. Therefore, over the past decade, extensive efforts have been made in order to provide alternative solutions for localization purposes.

Among several localization methods [376], multilateration is of great importance for precisely determining the users' positions. For this method, at least three estimations of the node's distance from three different anchor positions are needed. The distance estimation is typically done using time-based or (received signal strength)-received signal strength (RSS)based techniques [377]. In time-based techniques, the distance is estimated by multiplying the flight time by the light's speed. However, determining the flight's time is a tough challenge. This is because an adequately precise time synchronization is required between the transmitter and the receivers which is hard to acquire. However, time synchronization is not needed for an RSS-based solution. Moreover, RSS estimation functionality is available in the chipsets [378]. In the RSSbased method, the distance is estimated by using the RSSdistance function well represented by the path loss models.

RSS-based localization, however, faces an important challenge when the anchors are on the ground due to significant variation around the mean received signal power, as known as the shadowing effect. In effect, a ground-to-ground communication link suffers from a high level of shadowing which results in a large estimation error of the distance between a node, to be localized, and the ground anchor. This fact imposes a low localization accuracy and limits the use of the RSS-based method [378].

The characteristic of shadowing corresponding to different receiver's altitude has been studied in [379]. This shows that shadowing is less at higher altitude for ground-to-air communication, although the mean path loss behaves differently. Accordingly, the use of UAV anchors for RSS-based ground localization sounds like an interesting solution to mitigate the weakness of the RSS-based technique. In this manner, UAVs can act as aerial anchors to localize ground IoT devices. UAV anchors can combine the benefits of satellites with a good link probability of LOS and the advantages of ground anchors with a short link length and hence might result in a higher RSS resolution.

Several of the recent studies have addressed the concept of using UAVs for ground localization [366,380-382]. In [381] the authors considered the localization of IoT ground devices by using multiple static UAV anchors located at arbitrary locations. This study shows that the aerial anchors are capable of localizing the ground nodes with notably higher accuracy if the altitudes of the UAVs are adopted properly. Indeed, it is shown that the impact of UAVs altitudes is significant in an urban environment due to considerable variation of shadowing and mean power with the altitude. Statistically speaking, as the altitude of a UAV increases the communication link with the ground becomes more LOS and hence the mean path loss decreases. However, at a certain altitude at which the link is almost always LOS the more increase of the UAV altitude increases the path loss due to longer link length. We note that, in contrast to the mean path loss behavior, the shadowing, i.e. the fluctuation around the mean path loss, is becoming less 
at a higher altitude. In [381], the authors for the first time showed that in an urban area there exits an optimum altitude at which the localization accuracy is the highest.

The same report has investigated other important design factors effects such as UAVs' inter-distances and the number of UAVs. The results reveal that there is optimal UAVs interdistances for the maximum localization accuracy in order to localize ground IoT devices uniformly distributed over a certain region. Also, as the number of UAVs increases the localization error drops sharply.

The UAVs networking is constrained by their energy budget. As known well, the mechanical energy consumption of present UAVs are dominant over their electrical energy consumption and hence play a prominent role in their trajectory design and the lifetime of the network. Accordingly, it is of great importance to study such impact on the target quality of service. For this, first, a detailed description of UAV's energy consumption model is presented in [382], where it is shown that the velocity of UAV can be optimized for the minimum energy consumption. Interestingly, such an optimum velocity is not zero for a rotary-wing UAV and hence hovering the UAV may consume more energy than a mobile UAV. Then by taking such an issue into account, an optimized trajectory design of UAVs for minimum localization error is investigated. In addition, the data acquisition time and its role in the localization error have been studied.

3) IoT wireless energy supplier and information dissemination with UAVs: Limited energy budget and connectivity problem of IoT devices in certain environments and disaster cases can be well addressed using UAVs. Compared to fixed infrastructure UAVs could be more suitable for wireless power transfer (WPT) due to their flexible deployment and mobility which bring them to the proximity of IoT nodes. Furthermore, due to interference issues or limited battery capacity, the ground IoT transmitters may lower their transmit power resulting in a low range of communication for some cases. In such a situation, rapid deployment of UAVs can assist to broadcast common and private files.

A UAV trajectory for WPT is optimized in [383] where ground terminals use the received energy to upload their data to the UAV. In [384] a joint optimization of UAV trajectory and power control along with the communication scheduling of UAVs serving ground terminals maximizes the minimum rate of downlink communication. In [385-388], the authors study the deployment of UAVs for simultaneous data and energy transfer to IoT devices. For these, the trajectory and resource scheduling of UAVs are optimized in [385] to prolong the IoT network lifetime. The authors in [386] study simultaneous data and energy transfer to the IoT devices using UAVs. In this work, the UAV transmit power for each stream of IoT nodes and its trajectory are optimized to increase the minimum rate of communication with the IoT devices.

To enable such UAV-assisted IoT networking and to improve the network functionality, one needs to provide reliable and secure communication between drones and ground stations, particularly when drones require a beyond visual LOS tetherless connectivity for control and/or communication. This should be performed by a wireless technology suitable for the target UAV application.

To support UAVs in large scales, the existing point-to-point UAV communication with a ground station over an unlicensed band fails to satisfy the important criterion for UAV communication and networking [389]. Specifically, related to UAV command and control and a beyond visual LOS connectivity, a high coverage and continuous connectivity is needed to guarantee reliable control and tracking of human-driven or autonomous UAVs. In addition, low latency communication is essential to enable a robust remote control. Moreover, associated with payload communication, a high throughput communication link is to be established in order to exchange data and stream video signals. UAV's communication technology should also be secure to protect the data and should be capable of controlling interference. Additional features include system scalability for supporting the prompt growth of UAVs and regulation compliance. Cellular technology seems to be an adequate choice for satisfying the aforementioned requirements. The feasibility of using the existing cellular networks and the corresponding LTE and 5G technologies for UAVs connectivity have been investigated in [389-392].

\section{B. Satellite Integration into IoT Networks}

Satellite systems enable sustainable global coverage and hence significantly increase the IoT applications. A review of the NB-IoT and NTN integration in [364] investigates how the NB-IoT can be modified to support satellite networks for IoT services. In a densely deployed IoT networks, the use of satellite for IoT traffic offloading through low earth orbit (LEO) satellites is an alternative solution which is investigated in [393]. In [394] UAVs acting as gateways transfer the collected IoT data to the satellite. In this paper, the problem of limited resources in integrated ground-air-space IoT networks is considered. To deal with this problem, spectral efficiency is maximized through the optimization of the UAV bandwidth allocation and gateway selection. In [395] a mobile IoT node and its improved connectivity through the integration of UAV relay and satellite system are presented. Improved performance of the system is shown through the optimization of UAV trajectory and power allocation, under the UAV energy constraint.

Using the DRL approach an energy-efficient channel allocation algorithm in LEO satellite IoT is presented in [396]. An optimization problem is formulated in [397] in order to minimize the energy cost and latency affected by user association and resource allocation for computing and communication. To handle the complexity of the problem, the DRL technique is partially used to solve joint user association and offloading decision sub-problem.

\section{Remaining Challenges and Future Directions}

The IoT has already have a big impact on our society and new applications are being introduced almost on a daily basis. With a vision of global, 3D connectivity and with the help of leading technologies like AI, 5G and beyond cellular networks, and cloud and edge computing, the IoT is expected to produce increasingly promising results in every aspect of the world 
around us from health to agriculture and industry. To this end, several important research issues remain to be addressed in the future. We conclude our survey by discussing these challenges and pinpointing key problems that should be addressed in order to fully utilize the IoT to improve quality of life and generate new revenue.

\section{A. Limited Battery Lifetime}

With the introduction of NB-IoT technology, the expected battery lifetime of IoT devices has been significantly improved in comparison with the LTE networks. However, the preliminary performance analyses show that there is still a gap between the expected battery lifetime in NB-IoT networks and the $5 \mathrm{G}$ expectations $[90,99]$. This is mainly because the energy consumption in synchronization, connection establishment, resource scheduling, and waiting for the control signals from the BS are still there in the NB-IoT standardizations $[90,99]$. Potential directions for resolving this problem include (i) relaxing the resource reservation procedure for short-packet IoT communications, i.e. grant-free radio access [398]; wake-up receiver for IoT devices; and energy transfer to IoT devices and energy harvesting from environment, which are investigated in the sequel. Furthermore, Fig. 20 presents an overview of schemes reviewed in this work which aim at increasing battery lifetime of IoT devices.

1) Grant-free radio access: grant-free access (GFA) to radio resources has been already implemented in IoT technologies that operate over the unlicensed spectrum, such as SigFox and LoRaWAN [79]. In GFA, once a packet arrives at the device, it is transmitted without any handshaking, resource reservation, or authentication process. This type of access is opposed to the legacy grant-based access schemes in existing cellular networks. As part of the efforts shaping the $5 \mathrm{G} \mathrm{NR}$, the use of non-orthogonal multiple access schemes, including GFA, has attracted profound attention in recent years $[238,399,400]$. This is due to the fact that a recurrent element in various beyond $5 \mathrm{G}$ visions is to move towards zero-cost zero-energy IoT communications [401]. Then, as GFA can potentially offer reduced complexity and energy consumption for the end-devices, it could be a complementary access solution for IoT communications in future wireless access networks, along with the legacy grant-based access (GBA) schemes. Performance of communication with GFA has been investigated in a number of recent works [402405], and also in the context of the 3GPP [406]. In [402], GFA in massive MIMO systems has been investigated and the analytic expressions of success probability for conjugate and zero-forcing beamforming techniques have been derived. In [407], a novel distributed GFA scheme has been presented, in which, a cell is divided into different layers based on the pre-determined inter-layer received power difference from devices. Then, the transmission of each device is adapted to the layer that device belongs (close to the repetition class in the context of NB-IoT). The performance of a massive deployment of devices leveraging GFA has been investigated in [404], and approximate expressions for outage probability and throughput have been derived. Regarding the extra complexity needed in
GFA receivers, deep learning powered receiver for increasing the reliability of GFA systems has been investigated in [405]. Furthermore, ML-powered radio resource provisioning for hybrid grant-free/grant-based resource management has been investigated in [408]. In the context of the 3GPP, the set of radio resources that could be allocated to GFA communications, and the choice of modulation and coding scheme (MCS) have been investigated in [406]. Furthermore, regarding the fact that GFA is a complementary solution to the legacy GBA solution, the impact of GFA on GBA communications has been investigated in [406].

Challenges of GFA: In the following, we describe some unexplored research problems related to GFA which are investigated in this work.

a) Reliability of GFA: Once access to radio resources is shared among a set of devices which are sporadically active, the first concern to be addressed is the reliability of communications. Especially, when we consider wide-area IoT networks, supporting heterogeneous IoT devices, it is important to investigate the probability of success in grantfree communications for different transmission protocols of different traffic types. In [409], outage probability for GFA has been studied by assuming a constant received power from all contending devices. The success probability in grant-free radio access has been analyzed in $[410,411]$ by assuming a Poisson point process distribution for IoT devices over the service area. Performance of SJD and SIC in detection/decoding of collided packets in a grant-free IoT network has been investigated in [412]. Regarding the fact that reliability is amongst the main KPIs for communications, and GFA communications are vulnerable to collisions, more complex and advanced receivers, are expected to be explored in future research works [101, 398, 405]. Especially, an advanced receiver leveraging carrier frequency offset of IoT devices for collision decoding has been presented in $[101,398]$.

b) Coexistence analysis in GFA: For GFA IoT solutions, especially over the unlicensed spectrum, coexistence analysis is of crucial importance. For example, in the deployment phase of a new IoT service, when the choice of IoT connectivity solution, including LoRaWAN and SigFox, must be done, such analysis enables feasibility check for each connectivity option in presence of already existing IoT communications. The state-of-the-art research on performance analysis of GFA IoT networks has been mainly focused on performance analysis in homogeneous scenarios $[409,410]$, e.g. single technology case. Hence, there is a lack of research on performance analysis with multi-type IoT devices and heterogeneous communications characteristics, which is investigated here.

c) Scalability of GFA: Another important challenge with grant-free radio access IoT connectivity is the scalability analysis. The scalability analysis is indeed of crucial importance for IoT solutions over the licensed radio resources due to the scarcity of radio resources, and the crucial impact of the amount of allocated radio resources on the QoS of communications. For example, the scalability analysis can shed light on the extent up to which, QoS of communications could be preserved by increasing the number of devices that have access to the shared resource and is currently an active 
study item in 3GPP standardization [406]. Apart from the radio resource, the required density of the APs as well as the required device's consumed energy per packet ${ }^{10}$, is tightly coupled with the required QoS, and the rate of increase in each of them by increasing the QoS demand is of crucial importance. Therefore, there is a need to develop a model to investigate such a relation between the provisioned resources and QoS demand, which is investigated here.

d) Device operation control in GFA: Finally, in GFA, due to the reduced signaling between devices and the network, the fine-grained control over the operation of each device is missing. Then, tuning the communications parameters of devices $^{11}$, for achieving a level of QoS is challenging. For example, the analysis in $[101,398]$ presents that increasing the number of replicas per packet increases both reliability and energy efficiency of communications, beyond that level and up to the next level it tunes a tradeoff between them, and beyond the next level it deteriorates both of them. In contrast to such crucial impact, the operation control for GFA IoT networks is missing in the literature, and the state-of-the-art IoT networks usually use predetermined communication parameters, e.g. 3 replicas per packet in SigFox [79].

2) Wake-up receiver for IoT: Since most IoT devices are battery-driven, enhancing energy efficiency has been always a crucial KPI for network designers. A novel scheme to realize long-lasting IoT devices is to equip them with wakeup receivers (WuRs). A WuR is a module, integrated into the main circuit or along with that circuit, which can awake the device on-demand. WuRs usually consist of an RF demodulator, sequence decoder, digital address decoder, and are provided with a unique authentication address for paging [413]. The WUR constantly intercepts and decodes from a specific radio channel, transmitting the entire wake-up calls until it recognizes its unique address to awaken the device. The presence of an ultra-low-power WUR capable of harvesting its adequate energy from ambient sources such as wireless, thermal, light, and vibration energy is a promising method to maintain the preserved battery power and increase the device's lifespan. Towards this end, the energy consumption of the WuR circuit must be as low as possible. On the other hand, the reliability of WuRs, including (i) the risk of false alarm (making device awake when another device has been paged); and (ii) and risk of missed alarm (missing the paging from the network when data is waiting for the device or input from the device is needed), are to be investigated. Finally, although the literature on making efficient demodulators is mature, there is a lack of research on fast yet low-power and reliable address decoders. As this module is continuously monitoring the received ambient engines of the environment for potential paging of the device, and the IoT market demands longer device addresses for the sake of security and expansion of IoT networks, the contribution of energy consumption of address decoding to total WuR's energy consumption is of crucial importance [414].

\footnotetext{
${ }^{10}$ for transmit power or transmission of multiple replica

${ }^{11}$ including transmit power, data rate, spreading factor, and etc.
}

3) Energy harvesting and wireless power transfer for IoT: While replacing batteries of devices in general is time and cost consuming due to the need for human intervention, in some remote IoT applications, access to devices is limited. As an example, one can consider the multitude of IoT devices embed in today's modern airplanes, where most of devices are under the inner decoration of the aircraft. In order to avoid the difficulty in replacing the device or battery in such applications, harvesting energy is considered as a promising solution [415]. Broadly speaking, energy could be harvested from different natural sources, including thermal and solar, and radio signals like TV and radio broadcasting [416]. When a device relies on harvested energy as the main power source, its performances ties closely to the statistics of energy harvesting and utilization (battery-less operation). This is because for natural sources like solar and wind, it is difficult to predict the time and the amount of available energy. Hence, employing energy buffer, i.e., battery for energy storage, might be necessary to compensate the unpredictability of the energy. In contrast to the energy harvesting approach, wireless power transfer relies on intended radio signals, e.g. from the base station, for charging batteries of devices. In Section VIII-A3, prior art on WPT, especially through UAVs, has been presented. The major challenge with the above schemes, both from natural and radio transmissions, comes from the fact that the rate of saving energy by existing technologies are low in comparison to the rate of energy consumption in activity and data transmission of devices. Hence, devices need to store the harvested energy for a longer time, to a sufficient level, and during this time interval, devices won't be accessible by the network, and hence, it incurs delay in communications. Apart from these challenges, and if the potential delay in accessibility of the device is acceptable, the received energy from nature or base station can enable a device to last for a long time without human intervention. In [417], performance of energy harvesting IoT devices in random access over NB-IoT networks has been investigated. In [415], recent advances in energy harvesting for IoT have been presented, and future research challenges that must be addressed to realize the large-scale deployment of energy harvesting solutions for IoT have been investigated. An energy harvesting circuit has been presented in [418], which is compatible with the mmWave frequencies of $5 \mathrm{G}$ technologies. The spatio-temporal variations in the amount of harvested energy by IoT devices have been investigated in [416], and the challenges to be dealt in enabling wide usage of energy harvesting in NB-IoT networks have been presented.

\section{B. mmWave Access}

For future mmWave access, the choice of waveform design and multiple-access technique are among the major open problem. The challenges root in differences in hardware design in high frequencies. It is crucial for the realization of low-cost, efficient and reliable mmWave access to explore and innovate solutions based upon and beyond the existing ones, including OFDM-based and carrier-less UWB transmission.

In presence of analog beamforming with beam-based access, high-gain beams are known to be beneficial to the link budget 


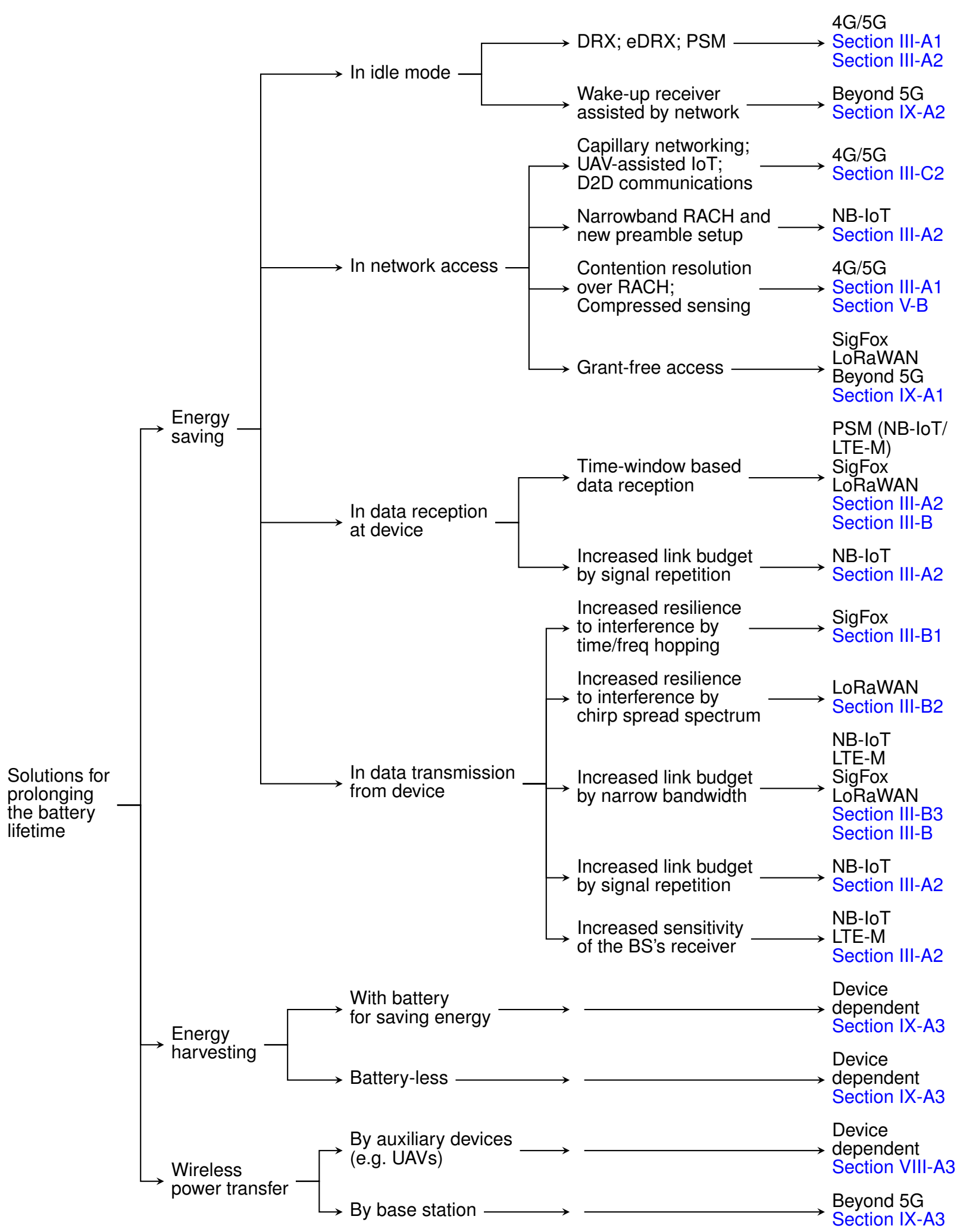

Fig. 20: Classification of various solutions targeting a long battery lifetime for IoT devices. 
and achievable data rate, however, they bring about some challenges too. For instance, current mmWave access points equipped with phased arrays can illuminate one 'beam' at a time, imposing a large delay in serving a multi-user network system, where the users are spatially spread and cannot be served by the same beam without loss of efficiency. Moreover, in dynamic IoT systems, for examples in a highly flexible factory floor deployment, tracking the best beam to serve each user becomes a tedious and time-consuming task with a large imposed overhead. Thus, there is a clear trade-off between the gain of narrow beams and the ease and rapidity of beam selection of wide beams. It is beneficial to jointly optimize the beam design and beam selection according to the dynamics of the network as well as the dynamics of the traffic.

Additionally, the development and use of intelligent reflective surfaces in shaping the propagation environment in high frequency bands, despite a large number of publications on the topic, is still a big open area of research. Especially in indoor deployments, such reflective surfaces have shown a great deal of potential for controlling the propagation environment [419]. Nevertheless, proper hardware development technology and algorithm design to deliver on the promise of low-cost lowenergy intelligent reflective surfaces require extensive research and development effort.

\section{Network-Device Cooperation}

The future research and development for adaptive networkdevice cooperative schemes, particularly in the context of URLLC use cases, is envisioned to pay more attention to the following areas.

1) Low-latency multi-hop transmission: Traditionally, relays have been considered as a means for extending coverage, less for low-latency applications. There is an inherent and unavoidable added latency from two-hop (and multihop) relaying. The main bottleneck for low-latency multi-hop relaying is in the processing (e.g., in case of decode-andforward relaying) that is expected at the relay nodes, which can take several milliseconds down to a fraction of a millisecond, depending on the hardware capabilities. With rapid advances in UE capability categories and coding algorithms, the processing times for decoding and re-encoding a packet are expected to become shorter. For example, one can easily confirm that a user plane latency of $1 \mathrm{~ms}$ over two-hop transmission is achievable with 3GPP Release 15 considerations for UE capabilities and radio access numerology. Deploying relaying techniques with less processing overhead, e.g., denoise-andforward relaying, can further reduce the expected delay per relaying hop [420]. Similarly, relaying delay can be reduced by full-duplex relaying operation [421].

2) Distributed cooperative transmission: To enable cooperative relaying from multiple devices in the network, distributed multi-antenna transmission schemes, such as distributed cyclic delay diversity (CDD) for OFDM modulation, are required [422]. In CDD, by applying conscious cyclic shifts to the OFDM symbol transmitted from each relay node, the spatial diversity from distributed relays is transformed into frequency diversity, which can be picked up by error-correcting codes such as the LDPC, which is utilized by $5 \mathrm{G}$ NR for data channels. Despite the extensive literature on such distributed cooperative transmission techniques, we expect to see emerging techniques to enable distributed and seamless cooperative transmission techniques that include on-the-run relay selection to improve energy efficiency and reduce interference footprint.

\section{Massive Connectivity}

The research on B5G cellular IoT has already begun and 6G is currently slated to enable a connection density of $10^{7}$ devices $/ \mathrm{km}^{2}$.

1) NOMA: In theory, downlink NOMA can be straightforwardly applied in almost any existing OMA-based solutions. However, several important challenges need to be addressed before adopting NOMA in IoT devices in practice. Two important issues are obtaining global, perfect CSI at each node and implementing SIC with acceptable complexity and error. Neither of the two is affordable in low-cost devices, so it is unlikely to have downlink NOMA in massive IoT applications. In fact, such limited-capacity devices cannot perform complex operations like SIC. Novel encoding/decoding techniques are to be designed before applying NOMA to mMTC applications. NOMA may also be used in broadband IoT and in general in high-capacity devices like cell phones. Even in this case, extensive experimental results are needed to prove the superiority of NOMA in practice where CSI is imperfect and SIC is prone to error propagation. Preliminary results on NOMA with imperfect CSI can be found in [423-425]. Besides, new overthe-air experiments of NOMA using software-defined radio as well as a list of existing literature and remaining challenges are reported in [426].

Uplink NOMA schemes are more likely to be adopted in the near future provided that the encoding complexity of codedomain NOMA schemes is reduced. Therefore, it is critically important to investigate low-complexity encoding schemes with an acceptable decoding error. Novel ML- and DL-based solutions can be developed to decrease the design complexity and increase performance. Applying such methods to massive access is expected to decrease the complexity of receivers.

2) Random access: In grant-free massive IoT, the BS is receiving data from a large number of uncoordinated devices. This results in resulting a high computational complexity at the BS. Another important issue in grant-free random access is device-activity detection. Approximate message passing algorithms [251-253] are proposed for this purpose. The application of DL is expected to decrease complexity further. More importantly, DL-aided solutions may bring noticeable gain for joint device detection and channel estimation as well as joint device and data detection. Overall, complexity reduction is the most crucial criterion for the wide adoption of any algorithm for massive connectivity and still requires intensive research. Finally, unsourced random access is a new promising approach toward massive connectivity [257], which deserves more investigation. 


\section{E. Channel Coding and Decoding}

Future wireless systems should provide service to various applications with a diverse range of requirements. For IoT, these requirements vary from high-reliability and low-latency to extreme low-energy consumption and deep coverage. Channel coding techniques need to designed to meet these requirements. We believe that the main focus for future wireless systems should be as follows:

1) Short packet communications: Most channel codes have been designed for long block lengths to meet Shannon's Limit. These codes are not working well under practical decoders at short block lengths. Although some designs have been proposed for polar and LDPC codes at short block lengths, they are not capable of supporting bit-level granularity of codeword length and rate. Puncturing and extending which are being commonly used for generating rate-compatible codes are sub-optimal which results in performance degradation at short block lengths. The design should be focused on developing novel techniques to generate rate-compatible codes with bitlevel granularity with an optimized performance at any desired rate and length. The code should also demonstrate a very low error floor. Rateless code and rate adaptation techniques can be potential candidates to solve this problem. LDPC codes over higher-order fields can be also a potential approach, however effective and low-complexity decoders should be designed. Moreover, with the cost of increased complexity, concatenated codes can be used to reduce the error floor. Moreover, some applications, like industrial IoT, require power burst error correction capability. Effective interleaving and deinterleaving approached should be designed for short packet communications.

2) Low-capacity and CSI-free communications: Many IoT applications will operate at an extreme-low power, which means that the received power is very low. The operating rate of the channel code is accordingly very low. Current channel codes, such as LDPC and polar codes, are unable to work efficiently at very low rates in the short block length regime. Therefore new channel codes that can operate at very low SNRs and be able to work efficiently with no/unreliable CSI are required. Moreover, these codes need the CSI to be available at the receiver to perform the decoding. At very low SNRs or when the CSI is not available at the receiver, the soft information is not accurate or reliable at the receiver; therefore, the decoding performance will dramatically degrade. Lowcomplexity OSD algorithms [178] can be a universal decoding technique that can work effectively under these conditions.

The bottleneck of the decoding in BCH-DFT is to find the position of errors. This is confirmed by assuming a genieaided error localization [172] which improves the performance improves largely. Hence, any solution improving the decoding will be a valuable contribution and can make this class of codes suitable for low latency communications. Further, these codes can be used for joint source-channel coding to further reduce the delay incurred in the communication system $[173,309]$. Another promising direction in this line is to seek methods that bypass the need for quantization of the codeword. If viable, such a solution will enjoy a much better decoding performance and can pave the road for widespread adoption of this class of codes in communication systems.

3) Decoder design: The end-to-end latency requirement of $0.5 \mathrm{~ms}$ of many low-latency applications, implies a physical layer latency of 8 to $67 \mu \mathrm{s}$. The channel decoder must share this latency budget with channel estimation and demodulation. Channel decoder should target processing latencies as low as $0.665 \mathrm{~s}$ [427]. This can be achieved by employing internal parallelism. The decoding techniques LDPC codes gain benefit from the parallel structure of the belief propagation decoding, which can be implemented efficiently. This provides low decoding latency and complexity. However, these decoders are sub-optimal for short packet communications. The decoding of polar codes mostly involves successive operations which introduces significant delays. It also involves large list sizes to achieve near-optimal performance, which requires large memory. These decoders are not efficient for resource-constraint IoT devices. The focus should be on using near-optimal decoders, such as OSD with sufficient and necessary conditions to reduce the complexity. These decoders are universal and can be applied to any linear code $[178,180,181]$. Further research is required to allow parallel computations to reduce the decoding run-time.

\section{F. Risk-aware Learning for IoT}

Introduction of mission-critical IoT into cellular networks mandates a transition from average-utility network design into risk-aware network design, where a rare event may incur a huge loss [408]. Instead of legacy ML algorithms, which aim at maximizing the average return of agents, or equivalently minimizing the average regret of agents, riskaware ML aims at minimizing the regret of huge loss due to potential performance degradation. Towards this end, extra emphasis must be put on the definition of a loss function. For example, consider risk-aware design of a traffic scheduler when serving solely one traffic type. In such a case, the scheduler design must enforce maximizing the first moment of the return, i.e. reliability, while minimizing the higher moments, e.g. the variance. Minimizing higher moments of the return, i.e. optimizing the worst-case return, could be achieved by defining the utility function as a nonlinear function of the return. A well-known example of a non-linear utility function is the exponential utility function [408], and works as follows. Considering latency $L$ as a KPI, the risk-aware scheduler design minimizes the expected value of $e^{L}$ instead of minimizing the expected value of $L$. In [408], a riskaware ML solution for radio resource management of nonscheduled URLLC traffic has been proposed. This solution enables safe delivery of short update packets by leveraging hybrid orthogonal/non-orthogonal radio resource slicing, and proactively regulating the spectrum needed for satisfying the required QoS.

A recent applications of risk-aware ML has emerged in the AoI research direction. Time-sensitive IoT applications, such as real-time monitoring by drone and terrestrial sensors, must rely on a timely status information delivery to the control entity. Then, the freshness of the received status updates at 
the control center is of crucial importance. The state-of-theart approaches in [428, 429] follow a risk-neutral approach, in which, the expected value of AoI cost functions is minimized. In order to capture the effect of rare events with a significant impact on the cost function, in [430], the notion of conditional value-at-risk for minimization of AoI for real-time IoT reporting has been proposed.

\section{G. Security Issues}

While Blockchains and SDN architectures can assist with some IoT security challenges, we are still struggling with effective key management. Keys and certificates are needed on a device to set up a secure channel to cloud services. Traditional ways of storing keys and certificates do not scale with the volume of IoT. An emerging solution would be to use a hardware-based authentication system that relies on the physical characteristics of the IoT device itself. These are inimitable properties unique to the hardware of each device that can not be modified, cannot be cloned and cannot be extracted. There are various types of physically unclonable functions (PUFs) that can assist in this domain, some examples are SRAM PUFs [431], butterfly PUFs [431], Ring oscillator PUFs [432], etc. This hardware-based technology provides a unique identity to every node (a very similar concept to a private key), which the node can use to authenticate as well as encrypt all data to protect data from the moment it leaves the device, all the way to the cloud services, and eventually achieve a true end-to-end protection.

\section{Conclusions}

IoT is playing an increasingly important role in the world around us and IoT market continues to grow at a fast pace. IoT connected to cellular and wide area networks are the fastest growing sectors of IoT, and current and new cellular standards are among the main enablers of various IoT applications. In this paper, we have provided a comprehensive survey of recent literature that lies at the intersection between IoT and 5G/6G cellular networks. We have listed and discussed the key performance indicators of $5 \mathrm{G}$ and $6 \mathrm{G}$ networks, indicated how IoT contributes to them and summarized recent advances maid along with those directions. In particular, we have described to what extent current research supports massive access, energy efficiency, reliability, latency, and security required for IoT, and what steps need to be taken to bridge the gap in the 2030s. This survey covers several aspects of IoT overlooked by previous surveys such as energy efficiency and channel coding for reliability and air latency. In addition, we have reviewed basic concepts and principles of various deep learning models and distributed and federated learning, emphasizing work specific to the IoT across different application scenarios. Further, we have discussed how to tailor deep learning models to IoT applications. We have also reviewed the solution for the integration of satellites and drones to next-generation IoT networks. We have concluded the survey by pinpointing several remaining challenges, open research issues, and promising directions, which may lead to valuable future research results. We hope that this survey becomes definite guide to researchers and developers interested in the IoT and a valuable resource for the field.

\section{REFERENCES}

[1] D. Evans, "The Internet of Things: How the next evolution of the Internet is changing everything," 2011, https://www.cisco.com/c/dam/ en_us/about/ac79/docs/innov/IoT_IBSG_0411FINAL.pdf.

[2] K. L. Lueth, "State of the IoT 2020: 12 billion IoT connections, surpassing non-IoT for the first time," 2020, https://iot-analytics. com/state-of-the-iot-2020-12-billion-iot-connections-surpassing-non iot-for-the-first-time/.

[3] "5G: The future of IoT," 2019, https://www.5gamericas.org/ wp-content/uploads/2019/07/5G_Americas_White_Paper_on_5G_ IOT_FINAL_7.16.pdf.

[4] Https://www.fortunebusinessinsights.com/industry-reports/internet-ofthings-iot-market-100307 [Online; accessed May 2021].

[5] M. Mohammadi, A. Al-Fuqaha, S. Sorour, and M. Guizani, "Deep learning for IoT big data and streaming analytics: A survey," IEEE Commun. Surveys Tuts., vol. 20, no. 4, pp. 2923-2960, 2018.

[6] E. AB, "Ericsson mobility report, November 2020," November 2020, https://www.ericsson.com/en/mobility-report/reports.

[7] "Cellular IoT evolution for industry digitalization," 2019, https://www.ericsson.com/en/reports-and-papers/white-papers/ cellular-iot-evolution-for-industry-digitalization.

[8] H. Lasi, P. Fettke, H.-G. Kemper, T. Feld, and M. Hoffmann, "Industry 4.0," Bus. Inf. Syst. Eng., vol. 6, no. 4, pp. 239-242, 2014.

[9] "The evolution of Industry 1.0 to 4.0," 2019, https://www.seekmomentum.com/blog/manufacturing/ the-evolution-of-industry-from-1-to-4.

[10] Deloitte, "Internet of Things (IoT): The rise of the connected world," 2019, www2.deloitte.com/content/dam/Deloitte/in/ Documents/technology-media-telecommunications/in-tmt-IoT_ Theriseoftheconnectedworld-28aug-noexp.pdf.

[11] A. Elnashar, "IoT evolution towards a super-connected world," arXiv:1907.02589, 2019.

[12] M. Kanj, V. Savaux, and M. Le Guen, "A Tutorial on NB-IoT physical layer design," IEEE Commun. Surveys Tuts., 2020.

[13] A. Hoglund, X. Lin, O. Liberg, A. Behravan, E. A. Yavuz, M. Van Der Zee, Y. Sui, T. Tirronen, A. Ratilainen, and D. Eriksson, "Overview of 3GPP release 14 enhanced NB-IoT," IEEE Netw., vol. 31, no. 6, pp. 16-22, 2017.

[14] Y.-P. E. Wang, X. Lin, A. Adhikary, A. Grovlen, Y. Sui, Y. Blankenship, J. Bergman, and H. S. Razaghi, "A primer on 3GPP narrowband Internet of Things," IEEE Commun. Mag., vol. 55, no. 3, pp. 117$123,2017$.

[15] A. Zaidi et al., "Designing for the future: the 5G NR physical layer," Ericsson Technology Review, 2017.

[16] I.-R. D. 5/40-E, "Minimum requirements related to technical performance for IMT-2020 radio interfaces," 2017, https://www.itu.int/pub/ R-REP-M.2410-2017.

[17] X. You, C.-X. Wang, J. Huang, X. Gao, Z. Zhang, M. Wang, Y. Huang, C. Zhang, Y. Jiang, J. Wang et al., "Towards $6 \mathrm{G}$ wireless communication networks: Vision, enabling technologies, and new paradigm shifts," China Inform. Sci., vol. 64, no. 1, pp. 1-74, 2021.

[18] H. Viswanathan and P. E. Mogensen, "Communications in the 6G era," IEEE Access, vol. 8, pp. 57 063-57 074, 2020.

[19] ITU, "Network 2030 - A blueprint of technology, applications and market drivers towards the year 2030 and beyond," 2021, https://www. itu.int/en/ITU-T/focusgroups/net2030/Documents/White_Paper.pdf.

[20] W. Shin and M. Vaezi, "UAV-enabled cellular networks," $5 G$ and Beyond: Fundamentals and Standards, pp. 165-200, 2021.

[21] X. Lin, V. Yajnanarayana, S. D. Muruganathan, S. Gao, H. Asplund, H.-L. Maattanen, M. Bergstrom, S. Euler, and Y.-P. E. Wang, "The sky is not the limit: LTE for unmanned aerial vehicles," IEEE Commun. Mag., vol. 56, no. 4, pp. 204-210, 2018.

[22] S. Tripathi, N. V. Sabu, A. K. Gupta, and H. S. Dhillon, "Millimeterwave and terahertz spectrum for 6G wireless," arXiv preprint arXiv:2102.10267, 2021.

[23] H. Haas, L. Yin, C. Chen, S. Videv, D. Parol, E. Poves, H. Alshaer, and M. S. Islim, "Introduction to indoor networking concepts and challenges in LiFi," J. Opt. Commun. Netw., vol. 12, no. 2, pp. A190A203, 2020.

[24] M. Z. Chowdhury, M. T. Hossan, A. Islam, and Y. M. Jang, “A comparative survey of optical wireless technologies: Architectures and applications," IEEE Access, vol. 6, pp. 9819-9840, 2018. 
[25] X. Lin and N. Lee, 5G and Beyond: Fundamentals and Standards. Springer Nature, 2021.

[26] S. Wijethilaka and M. Liyanage, "Survey on network slicing for Internet of Things realization in 5G networks," IEEE Commun. Surveys Tuts., vol. 23, no. 2, pp. 957-994, 2021.

[27] Y. Li et al., "Toward location-enabled IoT (LE-IoT): IoT positioning techniques, error sources, and error mitigation," IEEE Internet Things J., vol. 8, no. 6, pp. 4035-4062, 2021.

[28] R. A. Khalil, N. Saeed, M. Masood, Y. M. Fard, M.-S. Alouini, and T. Y. Al-Naffouri, "Deep learning in the industrial Internet of things: Potentials, challenges, and emerging applications," IEEE Internet Things J., 2021.

[29] F. Guo, F. R. Yu, H. Zhang, X. Li, H. Ji, and V. C. Leung, "Enabling massive IoT toward 6G: A comprehensive survey," IEEE Internet Things J, 2021.

[30] M. Stoyanova, Y. Nikoloudakis, S. Panagiotakis, E. Pallis, and E. K. Markakis, "A survey on the Internet of things (IoT) forensics: Challenges, approaches, and open issues," IEEE Commun. Surveys Tuts., vol. 22, no. 2, pp. 1191-1221, 2020.

[31] M. Elbayoumi, M. Kamel, W. Hamouda, and A. Youssef, "NOMAassisted machine-type communications in UDN: State-of-the-art and challenges," IEEE Commun. Surveys Tuts., vol. 22, no. 2, pp. 12761304, 2020.

[32] M. B. Shahab, R. Abbas, M. Shirvanimoghaddam, and S. J. Johnson, "Grant-free non-orthogonal multiple access for IoT: A survey," IEEE Commun. Surveys Tuts., vol. 22, no. 3, pp. 1805-1838, 2020.

[33] L. Lei, Y. Tan, K. Zheng, S. Liu, K. Zhang, and X. Shen, "Deep reinforcement learning for autonomous Internet of things: Model, applications and challenges," IEEE Commun. Surveys Tuts., vol. 22, no. 3, pp. $1722-1760,2020$.

[34] O. Maraqa, A. S. Rajasekaran, S. Al-Ahmadi, H. Yanikomeroglu, and S. M. Sait, "A survey of rate-optimal power domain NOMA with enabling technologies of future wireless networks," IEEE Commun. Surveys Tuts., vol. 22, no. 4, pp. 2192-2235, 2020.

[35] F. Hussain, R. Hussain, S. A. Hassan, and E. Hossain, "Machine learning in IoT security: Current solutions and future challenges," IEEE Commun. Surveys Tuts., vol. 22, no. 3, pp. 1686-1721, 2020.

[36] J. Qiu, Z. Tian, C. Du, Q. Zuo, S. Su, and B. Fang, "A survey on access control in the age of Internet of things," IEEE Internet Things J., vol. 7, no. 6, pp. 4682-4696, 2020.

[37] B. Buurman, J. Kamruzzaman, G. Karmakar, and S. Islam, "Low-power wide-area networks: Design goals, architecture, suitability to use cases and research challenges," IEEE Access, vol. 8, pp. 17 179-17 220, 2020.

[38] I. B. F. de Almeida, L. L. Mendes, J. J. Rodrigues, and M. A. da Cruz, "5G waveforms for IoT applications," IEEE Commun. Surveys Tuts., vol. 21, no. 3, pp. 2554-2567, 2019.

[39] L. Chettri and R. Bera, "A comprehensive survey on Internet of Things (IoT) toward 5G wireless systems," IEEE Internet Things J., vol. 7, no. 1, pp. 16-32, 2019.

[40] S. H. Alsamhi, O. Ma, M. S. Ansari, and Q. Meng, "Greening Internet of Things for greener and smarter cities: A survey and future prospects," Telecommun. Syst., vol. 72, no. 4, pp. 609-632, 2019.

[41] I. Stellios, P. Kotzanikolaou, M. Psarakis, C. Alcaraz, and J. Lopez, "A survey of IoT-enabled cyberattacks: Assessing attack paths to critical infrastructures and services," IEEE Commun. Surveys Tuts., vol. 20, no. 4, pp. 3453-3495, 2018.

[42] G. A. Akpakwu, B. J. Silva, G. P. Hancke, and A. M. Abu-Mahfouz, "A survey on 5G networks for the Internet of Things: Communication technologies and challenges," IEEE Access, vol. 6, pp. 3619-3647, 2017.

[43] J. Xu, J. Yao, L. Wang, Z. Ming, K. Wu, and L. Chen, "Narrowband Internet of things: Evolutions, technologies, and open issues," IEEE Internet Things J., vol. 5, no. 3, pp. 1449-1462, 2017.

[44] M. A. Ferrag et al., "Authentication protocols for Internet of things: A comprehensive survey," Secur. Commun. Netw., 2017.

[45] N. H. Motlagh, T. Taleb, and O. Arouk, "Low-altitude unmanned aerial vehicles-based Internet of things services: Comprehensive survey and future perspectives," IEEE Internet Things J., vol. 3, no. 6, pp. 899 922, 2016.

[46] A. Al-Fuqaha, M. Guizani, M. Mohammadi, M. Aledhari, and M. Ayyash, "Internet of things: A survey on enabling technologies, protocols, and applications," IEEE Commun. Surveys Tuts., vol. 17, no. 4, pp. 2347-2376, 2015.

[47] M. Series, "IMT vision-framework and overall objectives of the future development of IMT for 2020 and beyond," Recommendation ITU, vol. 2083, 2015.
[48] 5G-ACIA, "(white paper) 5G for automation in industry," 5G Alliance for Connected Industries and Automation, Tech. Rep., July 2019.

[49] "3GPP TS 22.104; service requirements for cyber-physical control applications in vertical domains," 3GPP, Tech. Spec., Dec. 2018.

[50] K. I. Pedersen, F. Frederiksen, G. Berardinelli, and P. E. Mogensen, "A flexible frame structure for 5G wide area," in Proc. IEEE Veh. Technol. Conf. (VTC-Fall), Sep. 2015.

[51] K. I. Pedersen, M. Niparko, J. Steiner, J. Oszmianski, L. Mudolo, and S. R. Khosravirad, "System level analysis of dynamic user-centric scheduling for a flexible 5G design," in Proc. IEEE Global Commun. Conf. (GLOBECOM), 2016, pp. 1-6.

[52] K. I. Pedersen, G. Pocovi, J. Steiner, and S. R. Khosravirad, "Punctured scheduling for critical low latency data on a shared channel with mobile broadband," in Proc. IEEE Veh. Technol. Conf. (VTC-Fall), 2017, pp. $1-6$.

[53] R. Jurdi, S. R. Khosravirad, H. Viswanathan, J. G. Andrews, and R. W. Heath, "Outage of periodic downlink wireless networks with hard deadlines," IEEE Trans. Commun., vol. 67, no. 2, pp. 1238-1253, Feb. 2019.

[54] K. I. Pedersen, S. R. Khosravirad, G. Berardinelli, and F. Frederiksen, "Rethink hybrid automatic repeat request design for 5G: Five configurable enhancements," IEEE Commun. Mag., 2017.

[55] N. H. Mahmood, A. Karimi, G. Berardinelli, K. I. Pedersen, and D. Laselva, "On the resource utilization of multi-connectivity transmission for URLLC services in 5G new radio," in Proc. IEEE Wireless Commun. Netw. Conf. (WCNC), 2019, pp. 1-6.

[56] "TS 22.263 Service requirements for video, imaging and audio for professional applications (VIAPA)," 3GPP, Technical Specification, Dec. 2020

[57] S. R. Khosravirad, G. Berardinelli, K. I. Pedersen, and F. Frederiksen, "Enhanced HARQ design for 5G wide area technology," in Proc. IEEE Veh. Technol. Conf. (VTC-Spring), May 2016.

[58] J. Park, S. Samarakoon, H. Shiri, M. K. Abdel-Aziz, T. Nishio, A. Elgabli, and M. Bennis, "Extreme URLLC: Vision, challenges, and key enablers," arXiv preprint arXiv:2001.09683, 2020.

[59] T. Adame, M. Carrascosa, and B. Bellalta, "Time-sensitive networking in IEEE 802.11 be: On the way to low-latency WiFi 7," arXiv preprint arXiv:1912.06086, 2019.

[60] 3GPP, "Study on physical layer enhancements for NR ultra-reliable and low latency case (URLLC)," Tech. Rep. TS 38.824, v. 2.0.1, Mar. 2019.

[61] H. Kokkoniemi-Tarkkanen et al., "Enabling safe wireless harbor automation via 5G URLLC," in Proc. IEEE 5G World Forum (5GWF), 2019, pp. 403-408.

[62] O. Tirkkonen, S. R. Khosravirad, P. Baracca, L. Zhou, U. Parts, D. Korpi, and M. Uusitalo, "Optimized survival mode to guarantee QoS for time-critical services," in Proc. IEEE Int. Commun. Conf. (ICC), Montreal, Canada, Jun. 2021.

[63] S. Samarakoon, M. Bennis, W. Saad, and M. Debbah, "Predictive ultra-reliable communication: A survival analysis perspective," IEEE Commun. Lett., vol. 25, no. 4, pp. 1221-1225, 2021.

[64] J. Demel, C. Bockelmann, and A. Dekorsy, "Burst error analysis of scheduling algorithms for 5G NR URLLC periodic deterministic communication," in Proc. IEEE Veh. Technol. Conf. (VTC-Spring), 2020, pp. 1-6.

[65] J. Gebert and A. Wich, "Alternating transmission of packets in dual connectivity for periodic deterministic communication utilising survival time," in Proc. European Conf. Netw. Commun. (EuCNC), 2020, pp. $160-164$.

[66] S. Paris, P. Kela, D. Laselva, and Q. Zhao, "Addressing reliability needs of industrial applications in 5G NR with network coding," in Proc. IEEE Veh. Technol. Conf. (VTC-Spring), 2020, pp. 1-6.

[67] S. Kaul, M. Gruteser, V. Rai, and J. Kenney, "Minimizing age of information in vehicular networks," in Proc. IEEE Conf. Sensor, Mesh, and Ad Hoc Commun. and Netw., 2011, pp. 350-358.

[68] M. K. Abdel-Aziz, C. Liu, S. Samarakoon, M. Bennis, and W. Saad, "Ultra-reliable low-latency vehicular networks: Taming the age of information tail," in Proc. IEEE Global Commun. Conf. (GLOBECOM), 2018, pp. 1-7.

[69] X. Zhang, Q. Zhu, and H. V. Poor, "Analyses for age of information supporting URLLC over multimedia wireless networks," in Proc. IEEE Int. Workshop Signal Process. Advances Wireless Commun. (SPAWC), 2020, pp. 1-5.

[70] I. Kadota, A. Sinha, and E. Modiano, "Optimizing age of information in wireless networks with throughput constraints," in Proc. IEEE Int. Conf. Comput. Commun. (INFOCOM), 2018, pp. 1844-1852. 
[71] Y. Hsu, E. Modiano, and L. Duan, "Age of information: Design and analysis of optimal scheduling algorithms," in Proc. IEEE Int. Symp. Inf. Theory (ISIT), 2017, pp. 561-565.

[72] A. Kosta, N. Pappas, and V. Angelakis, 2017.

[73] P. Popovski, F. Chiariotti, K. Huang, A. E. Kalør, M. Kountouris, N. Pappas, and B. Soret, "A perspective on time towards wireless 6G," 2021.

[74] 3GPP TSG GERAN 46, "USF capacity evaluation for MTC," Tech. Rep., May 2010.

[75] A. Azari and G. Miao, "Network lifetime maximization for cellularbased M2M networks," IEEE Access, vol. 5, pp. 18927-18940, Sept. 2017.

[76] X. Chen, D. W. K. Ng, W. Yu, E. G. Larsson, N. Al-Dhahir, and R. Schober, "Massive access for 5G and beyond," arXiv preprint arXiv:2002.03491, 2020.

[77] Ericsson, Huawei, NSN, and et al., "A choice of future M2M access technologies for mobile network operators," Tech. Rep., March 2014

[78] 3GPP TS 45.820, "Cellular system support for ultra-low complexity and low throughput Internet of Things (CIoT)," Tech. Rep., 2015, (Rel. 13).

[79] W. Yang et al., "Narrowband wireless access for low-power massive internet of things: A bandwidth perspective," IEEE Wireless Commun., vol. 24, no. 3, pp. 138-145, May 2017.

[80] A. I. Sulyman, S. M. Oteafy, and H. S. Hassanein, "Expanding the cellular-IoT umbrella: An architectural approach," IEEE Wireless Commun., vol. 24, no. 3, pp. 66-71, 2017.

[81] A. Azari, "Serving IoT communications over cellular networks: Challenges and solutions in radio resource management for massive and critical IoT communications," Ph.D. dissertation, KTH Royal Institute of Technology, 2018.

[82] G. Forecast, "Cisco visual networking index: global mobile data traffic forecast update, 2017-2022," Update, vol. 2017, p. 2022, 2019.

[83] R. Ratasuk, N. Mangalvedhe, and A. Ghosh, "Overview of LTE enhancements for cellular IoT," in Proc. IEEE Int. Symp. Pers. Indoor Mobile Radio-commun. (PIMRC), 2015, pp. 2293-2297.

[84] A. Azari et al., "Lifetime-aware scheduling and power control for M2M communications in LTE," in Proc. IEEE Veh. Technol. Conf. (VTCSpring), 2015

[85] M. Mehaseb, Y. Gadallah, A. Elhamy, and H. El-Hennawy, "Classification of LTE uplink scheduling techniques: An M2M perspective," IEEE Communications Surveys and Tutorials, no. 99, Nov. 2015.

[86] Nokia Networks, "LTE-M - optimizing LTE for the Internet of things," Tech. Rep., 2015.

[87] R. Ratasuk, B. Vejlgaard, N. Mangalvedhe, and A. Ghosh, "NB-IoT system for M2M communication," in Proc. IEEE Wireless Commun. Netw. Conf. (WCNC), Aug. 2016.

[88] Y. P. E. Wang et al., "A primer on 3GPP narrowband Internet of Things," IEEE Communications Magazine, vol. 55, no. 3, pp. 117123, March 2017.

[89] J. Schlienz and D. Raddino, "Narrowband Internet of Things," Rohde and Schwarz, Tech. Rep., Aug. 2016.

[90] P. A. Maldonado et al., "Narrowband IoT data transmission procedures for massive machine-type communications," IEEE Network, vol. 31, no. 6, pp. 8-15, Nov 2017.

[91] G. Association et al., "LTE-M deployment guide to basic feature set requirements," 2018

[92] A. K. Sultania, P. Zand, C. Blondia, and J. Famaey, "Energy modeling and evaluation of NB-IoT with PSM and eDRX," in Proc. IEEE Global Commun. Conf. Workshops (GC Workshops), 2018, pp. 1-7.

[93] A. K. Sultania, C. Blondia, and J. Famaey, "Optimizing the energylatency trade-off in NB-IoT with PSM and eDRX," IEEE Internet Things J., 2021.

[94] B. Ray, "LTE eDRX and PSM Explained for LTE-M1," https://www.link-labs.com/blog/lte-e-drx-psm-explained-for-lte-m1, accessed: 2021-06-09.

[95] X. Lin, A. Adhikary, and Y. P. E. Wang, "Random access preamble design and detection for 3GPP narrowband IoT systems," IEEE Wireless Communications Letters, vol. 5, no. 6, pp. 640-643, Dec. 2016.

[96] T. Kim, D. M. Kim, N. Pratas, P. Popovski, and D. K. Sung, "An enhanced access reservation protocol with a partial preamble transmission mechanism in NB-IoT systems," IEEE Communications Letters, June 2017.

[97] C. Yu et al., "Uplink scheduling and link adaptation for narrowband Internet of Things systems," IEEE Access, vol. 5, pp. 1724-1734, Feb. 2017.
[98] A. Adhikary, X. Lin, and Y. P. E. Wang, "Performance evaluation of NB-IoT coverage," in Proc. IEEE Veh. Technol. Conf. (VTC-Fall), Sept. 2016.

[99] A. Azari, G. Miao, C. Stefanovic, and P. Popovski, "Latency-energy tradeoff based on channel scheduling and repetitions in NB-IoT systems," Proc. IEEE Global Commun. Conf. (GLOBECOM), Dec. 2018.

[100] É. Morin, M. Maman, R. Guizzetti, and A. Duda, "Comparison of the device lifetime in wireless networks for the Internet of Things," IEEE Access, vol. 5, pp. 7097-7114, April 2017.

[101] A. Azari, P. Popovski, C. Stefanovic, and C. Cavdar, "Energy-efficient and reliable IoT access without radio resource reservation," IEEE Trans. Green Commun. Netw., 2021.

[102] M. Lauridsen et al., "Interference measurements in the european 868 MHz ISM band with focus on LoRa and SigFox," in Proc. IEEE Wireless Commun. Netw. Conf. (WCNC), March 2017.

[103] M. Lauridsen, "Studies on mobile terminal energy consumption for LTE, 5G," Ph.D. dissertation, Aalborg University, 2015.

[104] M. Centenaro et al., "Long-range communications in unlicensed bands: The rising stars in the IoT and smart city scenarios," IEEE Wireless Communications, vol. 23, no. 5, pp. 60-67, Nov. 2016.

[105] "NB-IoT vs. LoRa vs. Sigfox," https://www.link-labs.com/blog/ nb-iot-vs-lora-vs-sigfox, accessed: 2021-06-09.

[106] Semtech, "LoRa modulation basics," Tech. Rep., 2014. [Online]. Available: \url\{www.semtech.com\}

[107] N. Blenn and F. Kuipers, "LoRaWAN in the wild: Measurements from the things network," arXiv preprint arXiv:1706.03086, June 2017.

[108] D. Bankov, E. Khorov, and A. Lyakhov, "Mathematical model of LoRaWAN channel access," in Proc.IEEE 18th Int. Symposium World of Wireless, Mobile and Multimedia Netw., June 2017.

[109] _ - "On the limits of LoRaWAN channel access," in Proc. IEEE Int. Conf. Eng. Telecom., Nov. 2016.

[110] M. Lauridsen et al., "Coverage comparison of GPRS, NB-IoT, LoRa, and SigFox in a $7800 \mathrm{~km}^{2}$ area," in Proc. IEEE Veh. Technol. Conf. (VTC-Spring), 2017.

[111] F. Adelantado et al., "Understanding the limits of LoRaWAN," IEEE Commun. Mag, vol. 55, no. 9, pp. 34-40, 2017.

[112] F. C. Commission, "Use of spectrum bands above $24 \mathrm{GHz}$ for mobile radio services," Federal Communications Commission, Tech. Rep. 219 , 2016.

[113] M. R. Akdeniz, Y. Liu, M. K. Samimi, S. Sun, S. Rangan, T. S. Rappaport, and E. Erkip, "Millimeter wave channel modeling and cellular capacity evaluation," IEEE J. Sel. Areas Commun., vol. 32, no. 6, pp. 1164-1179, 2014

[114] S. K. Yoo, S. L. Cotton, R. W. Heath, and Y. J. Chun, "Measurements of the $60 \mathrm{GHz} \mathrm{UE}$ to eNB channel for small cell deployments," IEEE Wireless Commun. Lett., vol. 6, no. 2, pp. 178-181, 2017.

[115] D. Chizhik, J. Du, R. Feick, M. Rodriguez, G. Castro, and R. A. Valenzuela, "Path loss and directional gain measurements at $28 \mathrm{GHz}$ for non-line-of-sight coverage of indoors with corridors," IEEE Trans. Antennas Propag., vol. 68, no. 6, pp. 4820-4830, 2020.

[116] J. Du, D. Chizhik, R. Feick, G. Castro, M. Rodríguez, and R. A. Valenzuela, "Suburban residential building penetration loss at $28 \mathrm{GHz}$ for fixed wireless access," IEEE Wireless Commun. Lett., vol. 7, no. 6 , pp. 890-893, 2018.

[117] B. P. S. Sahoo, C. Chou, C. Weng, and H. Wei, "Enabling millimeterwave 5G networks for massive IoT applications: A closer look at the issues impacting millimeter-waves in consumer devices under the $5 \mathrm{G}$ framework," IEEE Commun. Lett., vol. 8, no. 1, pp. 49-54, 2019.

[118] T. K. Vu, C.-F. Liu, M. Bennis, M. Debbah, M. Latva-Aho, and C. S. Hong, "Ultra-reliable and low latency communication in mmwaveenabled massive MIMO networks," IEEE Commun. Lett., vol. 21, no. 9 , pp. 2041-2044, 2017.

[119] O. Semiari, W. Saad, M. Bennis, and M. Debbah, "Integrated millimeter wave and sub-6 GHz wireless networks: A roadmap for joint mobile broadband and ultra-reliable low-latency communications," IEEE Wireless Commun., vol. 26, no. 2, pp. 109-115, 2019.

[120] G. Berardinelli, N. H. Mahmood, I. Rodriguez, and P. Mogensen, "Beyond 5G wireless IRT for Industry 4.0: Design principles and spectrum aspects," in Proc. IEEE Global Commun. Conf. (GLOBECOM), Dec. 2018, pp. 1-6.

[121] M. S. Ibrahim, S. R. Khosravirad, J. Mazgula, H. Viswanathan, an N. D. Sidiropoulos, "Beam selection for Ultra-Reliable Low-Latency communication in industrial environment with beamforming repeaters," in Proc. IEEE Int. Commun. Conf. (ICC), Montreal, Canada, Jun. 2021.

[122] J. Mazgula, J. Sapis, U. S. Hashmi, and H. Viswanathan, "Ultra reliable low latency communications in mmWave for factory floor automation," Journal of the Indian Institute of Science, pp. 1-12, 2020. 
[123] M. Wu, D. Wuebben, A. Dekorsy, P. Baracca, V. Braun, and H. Halbauer, "Hardware impairments in millimeter wave communications using OFDM and SC-FDE," in Proc. 20th Int. ITG Workshop on Smart Antenn., 2016, pp. 1-8.

[124] V. Potdar, A. Sharif, and E. Chang, "Wireless sensor networks: A survey," in Proc. Int. Conf. Adv. Inform. Netw. Appl. Workshops, 2009, pp. 636-641.

[125] "3GPP TR 22.866; enhanced relays for energy efficiency and extensive coverage," 3GPP, Tech. Rep., Feb. 2019.

[126] M. S. Mekala and P. Viswanathan, "A survey: Smart agriculture IoT with cloud computing," in Proc. Int. conf. Microelectronic Devices, Circuits Syst. (ICMDCS), 2017, pp. 1-7.

[127] M. Mahmood, W. Seah, and I. Welch, "Reliability in wireless sensor networks: Survey and challenges ahead," Computer Networks, 012015.

[128] S. Murugaanandam and V. Ganapathy, "Reliability-based cluster head selection methodology using fuzzy logic for performance improvement in WSNs," IEEE Access, vol. PP, pp. 1-1, 072019.

[129] J. Zhang, J. Long, C. Zhang, and G. Zhao, "A delay-aware and reliable data aggregation for cyber-physical sensing," Sensors (Basel), vol. 17, p. 395, 022017.

[130] S. Lindsey, C. Raghavendra, and K. M. Sivalingam, "Data gathering algorithms in sensor networks using energy metrics," IEEE Trans. on Parallel and Distributed Systems, vol. 13, no. 9, pp. 924-935, 2002.

[131] J. Kruse, S. Mandelli, and S. R. Khosravirad, "QoS-aware wireless sensor networks: Reliability and low-latency for heterogeneous Industry 4.0," in Proc. IEEE VTC Spring, 2021, pp. 1-5.

[132] E. Dahlman, S. Parkvall, and J. Skold, 4G, LTE-advanced Pro and the Road to 5G. Academic Press, 2016.

[133] "3GPP TS 23.303; proximity-based services (prose)," 3GPP, Tech. Spec., Mar. 2016.

[134] "3GPP TR 36.746; study on further enhancements to LTE device to device (D2D), user equipment (UE) to network relays for Internet of Things (IoT) and wearables;", 3GPP, Tech. Rep., Apr. 2017.

[135] "3GPP TR 38.874; nr; study on integrated access and backhaul," 3GPP, Tech. Rep., Nov. 2018.

[136] N. H. Mahmood, H. Alves, O. A. López, M. Shehab, D. P. M. Osorio, and M. Latva-aho, "Six key enablers for machine type communication in 6G," arXiv preprint arXiv:1903.05406, 2019.

[137] R. Adeogun, G. Berardinelli, P. Mogensen, I. Rodriguez, and M. Razzaghpour, "Towards $6 \mathrm{G}$ in- $\mathrm{X}$ subnetworks with sub-millisecond communication cycles and extreme reliability," IEEE Access, 2020.

[138] L. Xu, R. Collier, and G. M. P. O?Hare, "A survey of clustering techniques in WSNs and consideration of the challenges of applying such to 5G IoT scenarios," IEEE Internet Things J., vol. 4, no. 5, pp. 1229-1249, Oct 2017.

[139] V. N. Swamy, S. Suri, P. Rigge, M. Weiner, G. Ranade, A. Sahai, and B. Nikolic, "Cooperative communication for high-reliability lowlatency wireless control," in Proc. IEEE Int. Commun. Conf. (ICC), June 2015, pp. 4380-4386.

[140] P. Zand, S. Chatterjea, K. Das, and P. Havinga, "Wireless industrial monitoring and control networks: The journey so far and the road ahead," Journal of sensor and actuator networks, vol. 1, no. 2, pp. 123-152, 2012.

[141] N. Brahmi, O. N. C. Yilmaz, K. W. Helmersson, S. A. Ashraf, and J. Torsner, "Deployment strategies for ultra-reliable and low-latency communication in factory automation," in Proc. IEEE GLOBECOM Workshops, Dec. 2015, pp. 1-6.

[142] B. M. Hochwald, T. L. Marzetta, and V. Tarokh, "Multiple-antenna channel hardening and its implications for rate feedback and scheduling," IEEE Trans. Inf. Theory, vol. 50, no. 9, pp. 1893-1909, Sep. 2004.

[143] S. A. Ayoughi, W. Yu, S. R. Khosravirad, and H. Viswanathan, "Interference mitigation for ultrareliable low-latency wireless communication," IEEE J. Sel. Areas Commun., pp. 1-1, 2019.

[144] S. R. Khosravirad, H. Viswanathan, and W. Yu, "Exploiting diversity for ultra-reliable and low-latency wireless control," IEEE Trans. Wireless Commun., vol. 20, no. 1, pp. 316-331, 2021.

[145] E. Arikan, "Channel polarization: A method for constructing capacityachieving codes for symmetric binary-input memoryless channels," IEEE Trans. Inf. Theory, vol. 55, no. 7, pp. 3051-3073, July 2009.

[146] R. Gallager, "Low-density parity-check codes," IRE Trans. Inf. Theory, vol. 8, no. 1, pp. 21-28, 1962.

[147] D. J. MacKay and R. M. Neal, "Near Shannon limit performance of low density parity check codes," Electronics letters, vol. 33, no. 6, pp. 457-458, 1997.
[148] T. J. Richardson and R. L. Urbanke, "The capacity of low-density parity-check codes under message-passing decoding," IEEE Trans. Inf. Theory, vol. 47, no. 2, pp. 599-618, 2001.

[149] M. Shirvanimoghaddam, M. S. Mohammadi, R. Abbas, A. Minja, C. Yue, B. Matuz, G. Han, Z. Lin, W. Liu, Y. Li et al., "Short blocklength codes for ultra-reliable low latency communications," IEEE Commun. Mag., vol. 57, no. 2, pp. 130-137, 2018.

[150] F. Abbasi, H. Mahdavifar, and E. Viterbo, "Polar coded repetition for low-capacity channels," arXiv preprint arXiv:2010.16144, 2020.

[151] M. Shirvanimoghaddam and S. Johnson, "Raptor codes in the low SNR regime," IEEE Trans. Commun., vol. 64, no. 11, pp. 4449-4460, 2016.

[152] H. Chen, R. Abbas, P. Cheng, M. Shirvanimoghaddam, W. Hardjawana, W. Bao, Y. Li, and B. Vucetic, "Ultra-reliable low latency cellular networks: Use cases, challenges and approaches," IEEE Commun. Mag., vol. 56, no. 12, pp. 119-125, 2018.

[153] A. Shokrollahi, "Raptor codes," IEEE Trans. Inf. Theory, vol. 52, no. 6, pp. 2551-2567, 2006.

[154] A. Gudipati and S. Katti, "Strider: Automatic rate adaptation and collision handling," SIGCOMM-Comput. Commun. Review, vol. 41, no. 4, pp. 158-169, 2011.

[155] H. Cui, C. Luo, K. Tan, F. Wu, and C. W. Chen, "Seamless rate adaptation for wireless networking," in Proc. ACM Int. Conf. Modeling, Analysis Simulation Wireless Mobile Syst., 2011, pp. 437-446.

[156] M. Shirvanimoghaddam, Y. Li, and B. Vucetic, "Near-capacity adaptive analog fountain codes for wireless channels," IEEE Commun. Lett., vol. 17, no. 12, pp. 2241-2244, 2013.

[157] J. Perry, P. A. Iannucci, K. E. Fleming, H. Balakrishnan, and D. Shah, "Spinal codes," ACM SIGCOM Comp. Commun. Review, vol. 42, no. 4, pp. 49-60, 2012.

[158] R. Abbas, M. Shirvanimoghaddam, T. Huang, Y. Li, and B. Vucetic, "Novel design for short analog fountain codes," IEEE Commun. Lett., vol. 23, no. 8, pp. 1306-1309, 2019.

[159] Z. Huang, J. Jiao, K. Zhang, S. Wu, and Q. Zhang, "A novel weight coefficient PEG algorithm for ultra-reliable short length analog fountain codes," IEEE Commun. Lett., vol. 8, no. 1, pp. 125-128, 2019.

[160] K. Zhang, J. Jiao, Z. Huang, S. Wu, and Q. Zhang, "Finite block-length analog fountain codes for ultra-reliable low latency communications," IEEE Trans. Commun., vol. 68, no. 3, pp. 1391-1404, 2020.

[161] X. Xu, S. Wu, D. Dong, J. Jiao, and Q. Zhang, "High performance short Polar codes: A concatenation scheme using Spinal codes as the outer code," IEEE Access, vol. 6, pp. 70644-70 654, 2018.

[162] R. Abbas, M. Shirvanimoghaddam, T. Huang, Y. Li, and B. Vucetic, "Performance analysis of short analog Fountain codes," in Proc. IEEE Global. Commun. Conf., 2019, pp. 1-6.

[163] Y. Polyanskiy, H. V. Poor, and S. Verdú, "Channel coding rate in the finite blocklength regime," IEEE Trans. Inf. Theory, vol. 56, no. 5, pp. 2307-2359, 2010.

[164] M. P. Fossorier and S. Lin, "Soft-decision decoding of linear block codes based on ordered statistics," IEEE Trans. Inf. Theory, vol. 41, no. 5, pp. 1379-1396, 1995.

[165] M. Shirvanimoghaddam, Y. Li, and B. Vucetic, "Near-capacity adaptive analog fountain codes for wireless channels," IEEE Commun. Lett., vol. 17, no. 12, pp. 2241-2244, Dec. 2013

[166] T. Marshall Jr., "Coding of real-number sequences for error correction: A digital signal processing problem," IEEE J. Sel. Areas Commun., vol. 2, no. 2, pp. 381-392, March 1984.

[167] J. K. Wolf, "Redundancy, the discrete Fourier transform, and impulse noise cancellation," IEEE Trans. Commun., vol. 31, no. 3, pp. 458-461, March 1983

[168] R. E. Blahut, Algebraic Codes for Data Transmission. New York: Cambridge University Press, 2003.

[169] G. Takos and C. N. Hadjicostis, "Determination of the number of errors in DFT codes subject to low-level quantization noise," IEEE Trans. Signal Process., vol. 56, no. 3, pp. 1043-1054, 2008.

[170] G. Rath and C. Guillemot, "Subspace algorithms for error localization with quantized DFT codes," IEEE Trans. Commun., vol. 52, no. 12, pp. 2115-2124, December 2004.

[171] M. Vaezi and F. Labeau, "Generalized and extended subspace algorithms for error correction with quantized DFT codes," IEEE Trans. Commun., vol. 62, no. 2, pp. 410-422, 2014.

[172] M. Vaezi, Distributed lossy source coding using BCH-DFT codes. Ph.D. dissertation, McGill University, 2014.

[173] A. Gabay, M. Kieffer, and P. Duhamel, "Joint source-channel coding using real $\mathrm{BCH}$ codes for robust image transmission," IEEE Trans. Signal Process., vol. 16, no. 6, pp. 1568-1583, June 2007. 
[174] G. Rath and C. Guillemot, "Subspace-based error and erasure correction with DFT codes for wireless channels," IEEE Trans. Signal Process., vol. 52, no. 11, pp. 3241-3252, November 2004.

[175] V. K. Goyal, J. Kovačević, and J. A. Kelner, "Quantized frame expansions with erasures," Appl. Comput. Harmon Anal., vol. 10, no. 3 , pp. 203-233, 2001.

[176] M. Vaezi and F. Labeau, "Systematic DFT frames: Principle, eigenvalues structure, and applications," IEEE Trans. Signal Process., vol. 61, no. 15 , pp. 3774-3885, August 2013.

[177] M. P. C. Fossorier and S. Lin, "Soft decision decoding of linear block codes based on ordered statistics," in Proc. IEEE Int. Symp. Inf. Theory (ISIT), Jun 1994, pp. 395-.

[178] C. Yue, M. Shirvanimoghaddam, B. Vucetic, and Y. Li, "A revisit to ordered statistics decoding: Distance distribution and decoding rules," IEEE Trans. Inf. Theory, pp. 1-1, 2021.

[179] C. Choi and J. Jeong, "Fast soft decision decoding of linear block codes using partial syndrome search," in Proc. IEEE Int. Symp. Inf. Theory (ISIT), 2020, pp. 384-388.

[180] C. Yue, M. Shirvanimoghaddam, Y. Li, and B. Vucetic, "Segmentationdiscarding ordered-statistic decoding for linear block codes," in Proc. IEEE Global Commun. Conf. (GLOBECOM), 2019, pp. 1-6.

[181] C. Yue, M. Shirvanimoghaddam, G. Park, O.-S. Park, B. Vucetic, and Y. Li, "Probability-based ordered-statistics decoding for short block codes," IEEE Commun. Lett., pp. 1-1, 2021.

[182] M. P. Fossorier, M. Mihaljevic, and H. Imai, "Reduced complexity iterative decoding of low-density parity check codes based on belief propagation," IEEE Trans. Commun., vol. 47, no. 5, pp. 673-680, 1999.

[183] J. Chen, A. Dholakia, E. Eleftheriou, M. P. Fossorier, and X.-Y. $\mathrm{Hu}$, "Reduced-complexity decoding of LDPC codes," IEEE Trans. Commun., vol. 53, no. 8, pp. 1288-1299, 2005.

[184] X. Wu, M. Jiang, and C. Zhao, "Decoding optimization for 5G LDPC codes by machine learning," IEEE Access, vol. 6, pp. 50 179-50 186, 2018.

[185] H. Cui, F. Ghaffari, K. Le, D. Declercq, J. Lin, and Z. Wang, "Design of high-performance and area-efficient decoder for 5G LDPC codes," IEEE Trans. Circuits Syst. I, 2020.

[186] K. Sun and M. Jiang, "A hybrid decoding algorithm for low-rate LDPC codes in 5G," in Proc. IEEE Int. Conf. Wireless Commun. Signal Process. (WCSP), 2018, pp. 1-5.

[187] K. Le Trung, F. Ghaffari, and D. Declercq, "An adaptation of minsum decoder for 5G low-density parity-check codes," in Proc. IEEE Int Symp Circuits Syst. (ISCAS), 2019, pp. 1-5.

[188] K. Chen, K. Niu, and J. Lin, "List successive cancellation decoding of Polar codes," Electronics letters, vol. 48, no. 9, pp. 500-501, 2012.

[189] K. Niu and K. Chen, "Stack decoding of polar codes," Electronics letters, vol. 48, no. 12, pp. 695-697, 2012.

[190] B. Li, H. Shen, and D. Tse, "An adaptive successive cancellation list decoder for Polar codes with cyclic redundancy check," IEEE Commun. Lett., vol. 16, no. 12, pp. 2044-2047, 2012.

[191] K. Niu and K. Chen, "CRC-aided decoding of Polar codes," IEEE Commun. Lett., vol. 16, no. 10, pp. 1668-1671, 2012.

[192] N. H. Mahmood, S. Böcker, A. Munari, F. Clazzer, I. Moerman, K. Mikhaylov, O. Lopez, O.-S. Park, E. Mercier, H. Bartz et al., "Critical and Massive Machine Type Communication Towards 6G," $6 G$ Channel, 2020

[193] M. Vaezi, Z. Ding, and H. V. Poor, Multiple Access Techniques for 5G Wireless Networks and Beyond. Springer, 2019.

[194] Cisco, "The zettabyte era: Trends and analysis," [Online; accessed September 2019], 2017, https://www.cisco.com/c/en/us/ solutions/collateral/service-provider/visual-networking-index-vni/ vni-hyperconnectivity-wp.pdf.

[195] 3GPP TR 38.812, "Study on non-orthogonal multiple access (NOMA) for NR," [Online]. Available: https://portal.3gpp.org/desktopmodules/ Specifications/SpecificationDetails.aspx?specificationId=3236.

[196] F. Kara and H. Kaya, "BER performances of downlink and uplink NOMA in the presence of SIC errors over fading channels," IET Commun., vol. 12, no. 15, pp. 1834-1844, 2018.

[197] W. Shin, H. Yang, M. Vaezi, J. Lee, and H. V. Poor, "Relay-aided NOMA in uplink cellular networks," IEEE Signal Process. Lett., vol. 24, no. 12, pp. 1842-1846, 2017.

[198] M. Zeng, W. Hao, O. A. Dobre, and H. V. Poor, "Energy-efficient power allocation in uplink mmWave massive MIMO with NOMA," IEEE Trans. Veh. Technol., vol. 68, no. 3, pp. 3000-3004, 2019.

[199] S. Schiessl, M. Skoglund, and J. Gross, "NOMA in the uplink: Delay analysis with imperfect CSI and finite-length coding," IEEE Trans. Wireless Commun., no. 6, pp. 3879-3893, 2020.
[200] Y. Yuan, "Industry Perspective: 5G non-orthogonal multiple access study," IEEE Commun. Mag., vol. 25, no. 5, pp. 4-6, October 2018.

[201] Y. Chen, A. Bayesteh, Y. Wu, B. Ren, S. Kang, S. Sun, Q. Xiong, C. Qian, B. Yu, Z. Ding et al., "Toward the standardization of nonorthogonal multiple access for next generation wireless networks," IEEE Commun. Mag., vol. 56, no. 3, pp. 19-27, 2018.

[202] L. Ping, L. Liu, K. Wu, and W. K. Leung, "Interleave division multipleaccess," IEEE Trans. Wireless Commun., vol. 5, no. 4, pp. 938-947, 2006.

[203] Hughes, "ACMA-OFDM for uplink NOMA, 3GPP TSG RAN WG1 92b,R1-1804171," April 2016, [Online]. Available: https://www.3gpp. org/DynaReport/TDocExMtg--R1-92b--18780.htm.

[204] Intel Corporation, "Multiple access schemes for new radio interface, 3GPP TSG-RAN WG1 84b,R1-162385," April 2016, [Online]. Available: https://www.3gpp.org/DynaReport/ TDocExMtg--R1-84b--31661.htm.

[205] S. Hu, B. Yu, C. Qian, Y. Xiao, Q. Xiong, C. Sun, and Y. Gao, "Nonorthogonal interleave-grid multiple access scheme for industrial Internet of things in 5G network," IEEE Trans. Ind. Informat., vol. 14, no. 12 , pp. 5436-5446, 2018.

[206] H. Nikopour, E. Yi, A. Bayesteh, K. Au, M. Hawryluck, H. Baligh, and J. Ma, "SCMA for downlink multiple access of 5G wireless networks," in Proc. IEEE Global Commun. Conf. (GLOBECOM), 2014, pp. 39403945.

[207] Nokia, Alcatel-Lucent, "Non-orthogonal multiple access for New Radio, 3GPP TSG-RAN WG1 85,R1-165019," May 2016, [Online]. Available: https://www.3gpp.org/DynaReport/ TDocExMtg--R1-85--31662.htm.

[208] LG Electronics, "Considerations on DL/UL multiple access for NR, 3GPP TSG-RAN WG1 84b,R1-162517," April 2016, [Online]. Available: https://www.3gpp.org/DynaReport/ TDocExMtg--R1-84b--31661.htm.

[209] Z. Yuan, G. Yu, W. Li, Y. Yuan, X. Wang, and J. Xu, "Multi-user shared access for Internet of things," in Proc. IEEE Veh. Technol. Conf. (VTCSpring), 2016, pp. 1-5.

[210] R. Hoshyar, F. P. Wathan, and R. Tafazolli, "Novel low-density signature for synchronous CDMA systems over AWGN channel," IEEE Trans. Signal Process., vol. 56, no. 4, pp. 1616-1626, 2008.

[211] R. Hoshyar, R. Razavi, and M. Al-Imari, "LDS-OFDM an efficient multiple access technique," in Proc. IEEE Veh. Technol. Conf. (VTCSpring), 2010, pp. 1-5.

[212] C. S. et al., "Pattern Division Multiple Access (PDMA). In: Vaezi M., Ding Z., Poor H. (eds) Multiple Access Techniques for 5G Wireless Networks and Beyond. Springer, Cham," 2019.

[213] ETRI, "Low code rate and signature based multiple access scheme for New Radio, 3GPP TSG RAN WG1 85,R1-164869," May 2016, [Online]. Available: https://www.3gpp.org/DynaReport/ TDocExMtg--R1-85--31662.htm.

[214] Qualcomm, "resource spread multiple access (RSMA), 3GPP TSG RAN WG1 85,R1-164688," May 2016, [Online]. Available: https: //www.3gpp.org/DynaReport/TDocExMtg--R1-85--31662.htm.

[215] R1-163510 , "Candidate NR multiple access schemes," Tech. Rep., April 2016, 3GPP TSG RAN WG1 Meeting 84, Korea.

[216] 3GPP TSG RAN WG1, "New uplink non-orthogonal multiple access schemes for NR," August 2016, [Online]. Available: https://www.3gpp. org/DynaReport/TDocExMtg--R1-86--31663.htm.

[217] S. Tang, L. Hao, and Z. Ma, "Low complexity joint MPA detection for downlink MIMO-SCMA," in Proc. IEEE Global Commun. Conf. (GLOBECOM), 2016, pp. 1-4.

[218] Z. Ma and J. Bao, Sparse Code Multiple Access (SCMA). Cham: Springer International Publishing, 2019, pp. 369-416.

[219] W.-C. Sun, Y.-C. Su, Y.-L. Ueng, and C.-H. Yang, "An LDPC-coded SCMA receiver with multi-user iterative detection and decoding," IEEE Trans. Circuits Syst., vol. 66, no. 9, pp. 3571-3584, 2019.

[220] J. Liu, G. Wu, S. Li, and O. Tirkkonen, "On fixed-point implementation of Log-MPA for SCMA signals," IEEE Wireless Commun. Lett., vol. 5, no. 3, pp. 324-327, 2016

[221] A. Ghaffari, M. Léonardon, A. Cassagne, C. Leroux, and Y. Savaria, "Toward high-performance implementation of 5G SCMA algorithms," IEEE Access, vol. 7, pp. 10402-10 414, 2019.

[222] T. M. Cover and J. A. Thomas, Elements of Information Theory. New York: John Wiley \& Sons, 2006.

[223] A. El Gamal and Y. H. Kim, Network Information Theory. Cambridge University Press, 2011.

[224] M. Vaezi and H. V. Poor, "NOMA: An information-theoretic perspective," in Multiple Access Techniques for 5G Wireless Networks and Beyond. Springer, 2019, pp. 167-193. 
[225] W. Shin, M. Vaezi, B. Lee, D. J. Love, J. Lee, and H. V. Poor, "Non-orthogonal multiple access in multi-cell networks: Theory, performance, and practical challenges," IEEE Commun. Mag., vol. 55, no. 10, pp. 176-183, 2017.

[226] 3GPP TD RP-150496, "Study on Downlink Multiuser Superposition Transmission," March 2015., [Online]. Available: https://portal.3gpp.org/desktopmodules/Specifications/ SpecificationDetails.aspx? specificationId $=2912$.

[227] Y. Yuan, Z. Yuan, G. Yu, C.-h. Hwang, P.-k. Liao, A. Li, and K. Takeda, "Non-orthogonal transmission technology in LTE evolution," IEEE Commun. Mag., vol. 54, no. 7, pp. 68-74, 2016.

[228] K. Y. Benjebbour A., Saito K., "Experimental Trials on NonOrthogonal Multiple Access," in Multiple Access Techniques for $5 G$ Wireless Networks and Beyond. Springer, 2019, pp. 587-607.

[229] S. Vanka, S. Srinivasa, Z. Gong, P. Vizi, K. Stamatiou, and M. Haenggi, "Superposition coding strategies: Design and experimental evaluation," IEEE Trans. Wireless Commun., vol. 11, no. 7, pp. 2628-2639, 2012.

[230] Y. Saito, Y. Kishiyama, A. Benjebbour, T. Nakamura, A. Li, and K. Higuchi, "Non-orthogonal multiple access (NOMA) for cellular future radio access," in Proc. IEEE Veh. Technol. Conf. (VTC-Spring), 2013, pp. 1-5.

[231] L. Dai, B. Wang, Y. Yuan, S. Han, C.-L. I, and Z. Wang, "Nonorthogonal multiple access for 5G: Solutions, challenges, opportunities, and future research trends," IEEE Commun. Mag., vol. 53, no. 9, pp. 74-81, 2015

[232] Y. Liu, Z. Qin, M. Elkashlan, Z. Ding, A. Nallanathan, and L. Hanzo, "Nonorthogonal multiple access for 5G and beyond," Proc. IEEE, vol. 105, no. 12, pp. 2347-2381, 2017.

[233] M. Vaezi, R. Schober, Z. Ding, and H. V. Poor, "Non-orthogonal multiple access: Common myths and critical questions," IEEE Wireless Commun., vol. 26, no. 5, pp. 174-180, 2019.

[234] M. Taherzadeh, H. Nikopour, A. Bayesteh, and H. Baligh, "SCMA codebook design," in Proc. IEEE Veh. Technol. Conf. (VTC-Fall), 2014, pp. $1-5$.

[235] A. Benjebbour, Y. Kishiyama, Y. Okumura, C.-H. Hwang, and I.-K $\mathrm{Fu}$, "Outdoor experimental trials of advanced downlink NOMA using smartphone-sized devices," in Proc. IEEE Veh. Technol. Conf. (VTCSpring), 2018, pp. 1-6.

[236] Q. Wu, W. Chen, D. W. K. Ng, and R. Schober, "Spectral and energyefficient wireless powered IoT networks: NOMA or TDMA?" IEEE Trans. Veh. Technol., vol. 67, no. 7, pp. 6663-6667, July 2018.

[237] Z. Ding, R. Schober, and H. V. Poor, "A general MIMO framework for NOMA downlink and uplink transmission based on signal alignment," IEEE Trans. Wireless Commun., vol. 15, no. 6, pp. 4438-4454, June 2016

[238] T. Lv, Y. Ma, J. Zeng, and P. T. Mathiopoulos, "Millimeter-wave NOMA transmission in cellular M2M communications for Internet of Things," IEEE Internet Things J., vol. 5, no. 3, pp. 1989-2000, 2018.

[239] M. A. Almasi, R. Amiri, M. Vaezi, and H. Mehrpouyan, "Lens-based millimeter wave reconfigurable antenna NOMA," in Proc. IEEE Int. Commun. Conf. Workshops (ICC Workshops), 2019, pp. 1-5.

[240] R. Jia, X. Chen, C. Zhong, D. W. K. Ng, H. Lin, and Z. Zhang, "Design of non-orthogonal beamspace multiple access for cellular Internet-ofThings," IEEE J. Sel. Topics Signal Process, vol. 13, no. 3, pp. 538552, 2019.

[241] M. Liu, T. Song, and G. Gui, "Deep cognitive perspective: Resource allocation for NOMA-based heterogeneous IoT with imperfect SIC," IEEE Internet Things J., vol. 6, no. 2, pp. 2885-2894, 2018

[242] K. N. Doan, M. Vaezi, W. Shin, H. V. Poor, H. Shin, and T. Q. Quek, "Power allocation in cache-aided NOMA systems: Optimization and deep reinforcement learning approaches," IEEE Trans. Commun., vol. 68 , no. 1 , pp. $630-644,2020$.

[243] G. Gui, H. Huang, Y. Song, and H. Sari, "Deep learning for an effective nonorthogonal multiple access scheme," IEEE Trans. Veh. Technol., vol. 67, no. 9, pp. 8440-8450, 2018.

[244] X. Zhang and M. Vaezi, "Deep learning based precoding for the MIMO Gaussian wiretap channel," in Proc. IEEE Global Commun. Conf. Workshops (GC Workshops), 2019, pp. 1-6.

[245] B. Clerckx, Y. Mao, R. Schober, E. Jorswieck, D. J. Love, J. Yuan, L. Hanzo, G. Y. Li, E. G. Larsson, and G. Caire, "Is NOMA efficient in multi-antenna networks? A critical look at next generation multiple access techniques," arXiv preprint arXiv:2101.04802, 2021.

[246] H. Weingarten, Y. Steinberg, and S. S. Shamai, "The capacity region of the Gaussian multiple-input multiple-output broadcast channel," IEEE Trans. Inf. Theory, vol. 52, no. 9, pp. 3936-3964, 2006.

[247] Y. Qi and M. Vaezi, "Secure transmission in MIMO-NOMA networks," IEEE Commun. Lett., vol. 24, no. 12, pp. 2696-2700, 2020.
[248] M. Vaezi and Y. Zhang, "Radio access network evolution," in Cloud Mobile Networks. Springer, 2017, pp. 67-86.

[249] A. Laya, L. Alonso, and J. Alonso-Zarate, "Is the random access channel of LTE and LTE-A suitable for M2M communications? A survey of alternatives," IEEE Commun. Surveys Tuts., vol. 16, no. 1 pp. 4-16, 2013.

[250] L. Liu, E. G. Larsson, W. Yu, P. Popovski, C. Stefanovic, and E. De Carvalho, "Sparse signal processing for grant-free massive connectivity: A future paradigm for random access protocols in the internet of things," IEEE Signal Process. Mag., vol. 35, no. 5, pp. $88-99,2018$.

[251] D. L. Donoho, A. Maleki, and A. Montanari, "Message-passing algorithms for compressed sensing," Proc. National Academy Sci., vol. 106 no. 45, pp. 18914-18919, 2009.

[252] L. Liu and W. Yu, "Massive connectivity with massive MIMO Part II: Achievable rate characterization," IEEE Trans. Signal Process. vol. 66, no. 11, pp. 2947-2959, 2018.

[253] M. Ke, Z. Gao, Y. Wu, X. Gao, and R. Schober, "Compressive sensingbased adaptive active user detection and channel estimation: Massive access meets massive MIMO," IEEE Trans. Signal Process., vol. 68, pp. 764-779, 2020.

[254] S. Li, W. Zhang, Y. Cui, H. V. Cheng, and W. Yu, "Joint design of measurement matrix and sparse support recovery method via deep autoencoder," IEEE Signal Process. Lett., vol. 26, no. 12, pp. 1778-1782, 2019.

[255] Y. Cui, S. Li, and W. Zhang, "Jointly sparse signal recovery and support recovery via deep learning with applications in MIMO-based grant-free random access," IEEE J. Sel. Areas Commun., 2020.

[256] J. Ding and J. Choi, "Comparison of preamble structures for grant-free random access in massive MIMO systems," IEEE Wireless Commun. Lett., vol. 9, no. 2, pp. 166-170, 2019.

[257] Y. Polyanskiy, "A perspective on massive random-access," in Proc. IEEE Int. Symp. Inf. Theory (ISIT), 2017, pp. 2523-2527.

[258] O. Ordentlich and Y. Polyanskiy, "Low complexity schemes for the random access Gaussian channel," in Proc. IEEE Int. Symp. Inf. Theory (ISIT), 2017, pp. 2528-2532.

[259] A. Fengler, G. Caire, P. Jung, and S. Haghighatshoar, "Massive MIMO unsourced random access," arXiv preprint arXiv:1901.00828, 2019.

[260] R. Calderbank and A. Thompson, "CHIRRUP: a practical algorithm for unsourced multiple access," Information and Inference: A Journal of the IMA, vol. 9, no. 4, pp. 875-897, 2020.

[261] G. Pathak, J. Gutierrez, and S. U. Rehman, "Security in low powered wide area networks: Opportunities for software defined network-supported solutions," Electron., vol. 9, no. 8, 2020. [Online]. Available: https://www.mdpi.com/2079-9292/9/8/1195

[262] P. A. Networks, "IoT threat report 2020," May 2020. [Online]. Available: https://unit42.paloaltonetworks.com/iot-threat-report-2020/

[263] C. Kolias, G. Kambourakis, A. Stavrou, and J. Voas, "DDoS in the IoT: Mirai and other botnets," Comput., vol. 50, no. 7, pp. 80-84, 2017.

[264] M. Wazid, A. K. Das, V. Odelu, N. Kumar, M. Conti, and M. Jo, "Design of secure user authenticated key management protocol for generic IoT networks," IEEE Int. of Things, vol. 5, no. 1, pp. 269 282, 2018.

[265] X. Yang, "LoRaWAN: Vulnerability analysis and practical expoitation," Master's Thesis, TU Delft Electrical Engineering, Mathematics and Computer Science, 2017.

[266] E. Aras, G. S. Ramachandran, P. Lawrence, and D. Hughes, "Exploring the security vulnerabilities of LoRa," in Proc. IEEE Int. Conf. on Cybern (CYBCONF), 2017, pp. 1-6.

[267] G. Ikrissi and T. Mazri, "A study of smart campus environment and its security attacks," ISPRS - Int. Arch. of the Photogramm., Remote Sens. and Spat. Inf. Sci., vol. XLIV-4/W3-2020, pp. 255-261, 112020.

[268] F. L. Coman, K. M. Malarski, M. N. Petersen, and S. Ruepp, "Security issues in Internet of things: Vulnerability analysis of LoRaWAN, Sigfox and NB-IoT," in Global IoT Summit (GIoTS), 2019, pp. 1-6.

[269] S. Chacko and M. D. Job, "Security mechanisms and vulnerabilities in LPWAN," IOP Conf. Series: Mater. Sci. and Eng., vol. 396 , p. 012027, aug 2018. [Online]. Available: https://doi.org/10.1088/ 1757-899x/396/1/012027

[270] Y. Gao, Y. Su, W. Yang, S. Chen, S. Nepal, and D. C. Ranasinghe, "Building secure SRAM PUF key generators on resource constrained devices," in Proc. IEEE Int. Conf. on Perv. Comput. and Commun. Wksp. (PerCom Workshops), 2019, pp. 912-917.

[271] E. Aras, N. J. Small, G. S. Ramachandran, S. Delbruel, W. Joosen, and D. Hughes, "Selective jamming of LoRaWAN using commodity hardware," CoRR, vol. abs/1712.02141, 2017. [Online]. Available: http://arxiv.org/abs/1712.02141 
[272] X. Yang, E. Karampatzakis, C. Doerr, and F. Kuipers, "Security vulnerabilities in LoRaWAN," in Proc. IEEE/ACM Int. Conf. on IoT Design and Impl. (IoTDI), 2018, pp. 129-140.

[273] G. Bernardinetti, F. Mancini, and G. Bianchi, "Disconnection attacks against LoRaWAN 1.0.X ABP devices," in Med. Commun. and Comput. Netw. Conf. (MedComNet), 2020, pp. 1-8.

[274] B. Xu, W. Wang, Q. Hao, Z. Zhang, P. Du, T. Xia, H. Li, and X. Wang, "A security design for the detecting of buffer overflow attacks in IoT device," IEEE Access, vol. PP, pp. 1-1, 112018.

[275] S. Shen, K. Zhang, Y. Zhou, and S. Ci, "Security in edgeassisted Internet of things: challenges and solutions," Sci. China Inf. Sci., vol. 63, no. 12, p. 220302, 2020. [Online]. Available: https://doi.org/10.1007/s11432-019-2906-y

[276] H. A. Abdul-Ghani, D. Konstantas, and M. Mahyoub, "A comprehensive IoT attacks survey based on a building-blocked reference model," Int. J. of Adv. Comput. Sci. and Appl., vol. 9, 2018.

[277] M. Davis, The Undecidable: Basic Papers on Undecidable Propositions, Unsolvable Problems, and Computable Functions. Dover Publication, 1965.

[278] P. Rost, C. Mannweiler, D. S. Michalopoulos, C. Sartori, V. Sciancalepore, N. Sastry, O. Holland, S. Tayade, B. Han, D. Bega, D. Aziz, and H. Bakker, "Network slicing to enable scalability and flexibility in $5 \mathrm{G}$ mobile networks," IEEE Commun. Mag., vol. 55, no. 5, pp. 72-79, 2017

[279] L. Wallgren, S. Raza, and T. Voigt, "Routing attacks and countermeasures in the RPL-based Internet of things," Int. J. of Distrib. Sens. Netw., vol. 9, no. 8, p. 794326, 2013. [Online]. Available: https://doi.org/10.1155/2013/794326

[280] D. Rupprecht, K. Kohls, T. Holz, and C. Pöpper, "Breaking LTE on layer two," in Proc. IEEE Symp. on Security and Privacy (SP), 2019, pp. 1121-1136.

[281] D. Rupprecht, K. Kohls, T. Holz, and C. Pöpper, "IMP4GT: Impersonation attacks in 4G networks," in Proc. ISOC Netw. and Distrib. Syst. Security Symp. (NDSS). ISOC, Feb. 2020

[282] D. E. Kouicem, A. Bouabdallah, and H. Lakhlef, "Internet of things security: A top-down survey," Comp. Netw., vol. 141, pp. 199-221, 2018. [Online]. Available: https://www.sciencedirect.com/ science/article/pii/S1389128618301208

[283] P. Hu, "A system architecture for software-defined industrial Interne of things," in IEEE Int. Conf. on Ubiquitous Wireless Broadband (ICUWB), 2015, pp. 1-5.

[284] O. Flauzac, C. González, A. Hachani, and F. Nolot, "SDN based architecture for IoT and improvement of the security," in Proc. IEEE Int. Conf. on Adv. Inf. Netw. and Appl. Wksp., 2015, pp. 688-693.

[285] A. Molina Zarca, J. B. Bernabe, R. Trapero, D. Rivera, J. Villalobos, A. Skarmeta, S. Bianchi, A. Zafeiropoulos, and P. Gouvas, "Security management architecture for NFV/SDN-aware IoT systems," IEEE Int. of Things, vol. 6, no. 5, pp. 8005-8020, 2019.

[286] A. Yazdinejad, R. M. Parizi, A. Dehghantanha, Q. Zhang, and K.K. R. Choo, "An energy-efficient SDN controller architecture for IoT networks with blockchain-based security," IEEE Trans. Services Comput., vol. 13, no. 4, pp. 625-638, 2020.

[287] B. Al-Hayajneh and McAndrew, "Improving Internet of things (IoT) security with software-defined networking (SDN)," Comp., vol. 9, p. 8, 022020.

[288] N. Ravi and S. M. Shalinie, "Learning-driven detection and mitigation of DDoS attack in IoT via SDN-cloud architecture," IEEE Int. of Things, vol. 7, no. 4, pp. 3559-3570, 2020.

[289] T. G. Nguyen, T. V. Phan, B. T. Nguyen, C. So-In, Z. A. Baig, and S. Sanguanpong, "Search: A collaborative and intelligent NIDS architecture for SDN-based cloud IoT networks," IEEE Access, vol. 7, pp. $107678-107694,2019$.

[290] M. Casado, M. J. Freedman, J. Pettit, J. Luo, N. McKeown, and S. Shenker, "Ethane: Taking control of the enterprise," SIGCOMM Comput. Commun. Rev., vol. 37, no. 4, p. 1-12, Aug. 2007. [Online]. Available: https://doi.org/10.1145/1282427.1282382

[291] N. McKeown, T. Anderson, H. Balakrishnan, G. Parulkar, L. Peterson, J. Rexford, S. Shenker, and J. Turner, "Openflow: Enabling innovation in campus networks," SIGCOMM Comput. Commun. Rev., vol. 38, no. 2, p. 69-74, Mar. 2008. [Online]. Available: https://doi.org/10.1145/1355734.1355746

[292] P. Bull, R. Austin, E. Popov, M. Sharma, and R. Watson, "Flow based security for IoT devices using an SDN gateway," in Proc. IEEE Int. Conf. on Future Int. of Things and Cloud (FiCloud), 2016, pp. 157163.

[293] R. Vilalta, R. Ciungu, A. Mayoral, R. Casellas, R. Martinez, D. Pubill, J. Serra, R. Munoz, and C. Verikoukis, "Improving security in Internet of things with software defined networking," in Proc. IEEE Global Commun. Conf. (GLOBECOM), 2016, pp. 1-6.

[294] B. Arshdeep and M. Vijay, "Blockchain platform for industrial Internet of things," J. of Softw. Eng. and Appl., vol. 09, pp. 533-546, 012016.

[295] M. A. Khan and K. Salah, "IoT security: Review, blockchain solutions, and open challenges," Future Gener. Comput. Syst., vol. 82, pp. 395-411, 2018. [Online]. Available: https://www.sciencedirect.com/ science/article/pii/S0167739X17315765

[296] A. Chaer, K. Salah, C. Lima, P. P. Ray, and T. Sheltami, "Blockchain for 5G: Opportunities and challenges," in Proc. IEEE Global Commun. Conf. (GLOBECOM), 2019, pp. 1-6.

[297] B. Xu, N. Wang, T. Chen, and M. Li, "Empirical evaluation of rectified activations in convolutional network," arXiv:1505.00853, 2015.

[298] Y. LeCun, Y. Bengio, and G. Hinton, "Deep learning," nature, vol. 521, no. 7553 , pp. 436-444, 2015.

[299] C. Zhang, P. Patras, and H. Haddadi, "Deep learning in mobile and wireless networking: A survey," IEEE Commun. Surveys Tuts., vol. 21, no. 3, pp. 2224-2287, 2019.

[300] M. A. Alsheikh, D. Niyato, S. Lin, H.-P. Tan, and Z. Han, "Mobile big data analytics using deep learning and apache spark," IEEE Netw., vol. 30, no. 3, pp. 22-29, 2016.

[301] P. Georgiev, S. Bhattacharya, N. D. Lane, and C. Mascolo, "Lowresource multi-task audio sensing for mobile and embedded devices via shared deep neural network representations," Proc. ACM Interact. Mob. Wearable Ubiquitous Technol., vol. 1, no. 3, pp. 1-19, 2017.

[302] X. Zhang and M. Vaezi, "Multi-objective DNN-based precoder for MIMO communications," IEEE Trans. Commun., 2021.

[303] T. J. O'Shea, T. Erpek, and T. C. Clancy, "Deep learning based MIMO communications," arXiv preprint arXiv:1707.07980, 2017

[304] Z. Zhang and A. Z. Kouzani, "Implementation of DNNs on IoT devices," Neural. Comput. Appl., vol. 32, no. 5, pp. 1327-1356, 2020.

[305] R. Hadidi, J. Cao, M. S. Ryoo, and H. Kim, "Toward collaborative inferencing of deep neural networks on Internet-of-Things devices," IEEE Trans. Cogn. Commun. Netw., vol. 7, no. 6, pp. 4950-4960, 2020.

[306] L. Deng, G. Li, S. Han, L. Shi, and Y. Xie, "Model compression and hardware acceleration for neural networks: A comprehensive survey," Proc. IEEE, vol. 108, no. 4, pp. 485-532, 2020.

[307] Z. Xiong, A. D. Liveris, and S. Cheng, "Distributed source coding for sensor networks," IEEE Signal Process. Mag., vol. 21, no. 5, pp. $80-94,2004$

[308] S. S. Pradhan and K. Ramchandran, "Distributed source coding using syndromes (DISCUS): Design and construction," IEEE Trans. Inf. Theory, vol. 49, no. 3, pp. 626-643, 2003.

[309] M. Vaezi and F. Labeau, "Distributed source-channel coding based on real-field BCH codes," IEEE Trans. Signal Process., vol. 62, no. 5, pp. 1171-1184, 2014.

[310] V. Chandola, A. Banerjee, and V. Kumar, "Anomaly detection: A survey," ACM comput. surveys (CSUR), vol. 41, no. 3, pp. 1-58, 2009.

[311] R. Chalapathy and S. Chawla, "Deep learning for anomaly detection: A survey," arXiv preprint arXiv:1901.03407, 2019.

[312] B. B. Zarpelão, R. S. Miani, C. T. Kawakani, and S. C. de Alvarenga, "A survey of intrusion detection in Internet of things," J. Netw. Comput Appl., vol. 84, pp. 25-37, 2017.

[313] G. Aceto, D. Ciuonzo, A. Montieri, and A. Pescapé, "Mobile encrypted traffic classification using deep learning: Experimental evaluation, lessons learned, and challenges," IEEE Trans. Netw. Service Manage., vol. 16, no. 2, pp. 445-458, 2019.

[314] T. Chen, X. Liu, B. Xia, W. Wang, and Y. Lai, "Unsupervised anomaly detection of industrial robots using sliding-window convolutional variational autoencoder," IEEE Access, vol. 8, pp. $47072-47$ 081, 2020.

[315] D. Azzalini, L. Bonali, and F. Amigoni, "A minimally supervised approach based on variational autoencoders for anomaly detection in autonomous robots," IEEE Robot. Autom. Lett., 2021.

[316] S. Nazir, S. Patel, and D. Patel, "Autoencoder based anomaly detection for SCADA networks," Int. J. Artif. Intell. Mach. Learn. (IJAIML), 2020

[317] C. Yin, S. Zhang, J. Wang, and N. N. Xiong, "Anomaly detection based on convolutional recurrent autoencoder for IoT time series," IEEE Trans. Syst. Man Cybern. Syst., 2020.

[318] M. Savic, M. Lukic, D. Danilovic, Z. Bodroski, D. Bajovic, I. Mezei, D. Vukobratovic, S. Skrbic, and D. Jakovetic, "Deep learning anomaly detection for cellular IoT with applications in smart logistics," arXiv preprint arXiv:2102.08936, 2021

[319] A.-H. Muna, N. Moustafa, and E. Sitnikova, "Identification of malicious activities in industrial Internet of things based on deep learning models," J. Inf. Secur. Appl., vol. 41, pp. 1-11, 2018. 
[320] Y. Meidan, M. Bohadana, Y. Mathov, Y. Mirsky, A. Shabtai, D. Breitenbacher, and Y. Elovici, "N-baiot-network-based detection of IoT botnet attacks using deep autoencoders," IEEE Pervasive Comput., vol. 17 , no. 3, pp. 12-22, 2018.

[321] S. J. Lee, P. D. Yoo, A. T. Asyhari, Y. Jhi, L. Chermak, C. Y. Yeun, and K. Taha, "IMPACT: Impersonation attack detection via edge computing using deep autoencoder and feature abstraction," IEEE Access, vol. 8, pp. 65 520-65 529, 2020.

[322] C. Kolias, G. Kambourakis, A. Stavrou, and S. Gritzalis, "Intrusion detection in 802.11 networks: empirical evaluation of threats and a public dataset," IEEE Commun. Surveys Tuts., vol. 18, no. 1, pp. 184208, 2015 .

[323] R.-H. Hwang, M.-C. Peng, and C.-W. Huang, "Detecting IoT malicious traffic based on autoencoder and convolutional neural network," in Proc. IEEE Global Commun. Conf. Workshops (GC Workshops), 2019, pp. 1-6.

[324] M. Al-Qatf, Y. Lasheng, M. Al-Habib, and K. Al-Sabahi, "Deep learning approach combining sparse autoencoder with SVM for network intrusion detection," IEEE Access, vol. 6, pp. 52 843-52 856, 2018.

[325] Y. Li, "Deep reinforcement learning: An overview," arXiv preprint arXiv:1701.07274, 2017.

[326] S. Gu, E. Holly, T. Lillicrap, and S. Levine, "Deep reinforcement learning for robotic manipulation with asynchronous off-policy updates," in Proc. IEEE int. conf. Robot. Autom. (ICRA), 2017, pp. 3389-3396.

[327] S. Chinchali, P. Hu, T. Chu, M. Sharma, M. Bansal, R. Misra, M. Pavone, and S. Katti, "Cellular network traffic scheduling with deep reinforcement learning," in Proc. AAAI Conf. Artif. Intell., vol. 32, no. $1,2018$.

[328] H. Li, K. Ota, and M. Dong, "Deep reinforcement scheduling for mobile crowdsensing in fog computing," ACM Trans. Internet Technol., vol. 19, no. 2, pp. 1-18, 2019.

[329] M. Liu, F. R. Yu, Y. Teng, V. C. Leung, and M. Song, "Performance optimization for blockchain-enabled industrial Internet of Things (IIoT) systems: A deep reinforcement learning approach," IEEE Trans. Ind. Informat., vol. 15, no. 6, pp. 3559-3570, 2019.

[330] H. Mao, M. Alizadeh, I. Menache, and S. Kandula, "Resource management with deep reinforcement learning," in Proc. ACM workshop hot topics netw., 2016, pp. 50-56.

[331] Y. Li, F. Qi, Z. Wang, X. Yu, and S. Shao, "Distributed edge computing offloading algorithm based on deep reinforcement learning," IEEE Access, vol. 8, pp. 85 204-85 215, 2020.

[332] F. Bu and X. Wang, "A smart agriculture IoT system based on deep reinforcement learning," Future Gener. Comput. Syst., vol. 99, pp. 500507, 2019.

[333] A. Azari, F. Ghavimi, M. Ozger, R. Jantti, and C. Cavdar, "Machine learning assisted handover and resource management for cellular connected drones," in Proc. IEEE Veh. Technol. Conf. (VTC-Spring), 2020, pp. 1-7.

[334] L. Clavier et al., "Experimental evidence for heavy tailed interference in the IoT," IEEE Commun. Lett., 2020.

[335] S. K. Sharma and X. Wang, "Collaborative distributed Q-learning for RACH congestion minimization in cellular IoT networks," IEEE Commun. Lett., vol. 23, no. 4, pp. 600-603, 2019.

[336] A. Azari, "Energy-efficient scheduling and grouping for machine-type communications over cellular networks," Ad Hoc Netw. J., vol. 43, pp. $16-29,2016$

[337] A. Azari and C. Cavdar, "Self-organized low-power IoT networks: A distributed learning approach," Proc. IEEE Global Commun. Conf. (GLOBECOM), 2018.

[338] D.-T. Ta, K. Khawam, S. Lahoud, C. Adjih, and S. Martin, "LoRaMAB: Toward an intelligent resource allocation approach for LoRAWAN," in Proc. IEEE Global Commun. Conf. (GLOBECOM), 2019, pp. $1-6$.

[339] R. M. Sandoval, A.-J. Garcia-Sanchez, and J. Garcia-Haro, "Optimizing and updating LoRa communication parameters: A machine learning approach," IEEE Trans. Netw. Service Manag, vol. 16, no. 3, pp. 884895, 2019.

[340] B. McMahan, E. Moore, D. Ramage, S. Hampson, and B. A. y Arcas, "Communication-efficient learning of deep networks from decentralized data," in Proc. Artif. Intell. Statist., 2017, pp. 1273-1282.

[341] M. Vaezi and Y. Zhang, Cloud Mobile Networks From RAN to EPC. Springer, 2017.

[342] A. Checko, H. L. Christiansen, Y. Yan, L. Scolari, G. Kardaras, M. S. Berger, and L. Dittmann, "Cloud RAN for mobile networks-A technology overview," IEEE Commun. Surveys Tuts., vol. 17, no. 1, pp. 405-426, 2014.
[343] B. Liu, L. Wang, M. Liu, and C.-Z. Xu, "Federated imitation learning: A novel framework for cloud robotic systems with heterogeneous sensor data," IEEE Robot. Autom. Lett., vol. 5, no. 2, pp. 3509-3516, 2020.

[344] B. Yin, H. Yin, Y. Wu, and Z. Jiang, "FDC: A secure federated deep learning mechanism for data collaborations in the Internet of Things," IEEE Trans. Cogn. Commun. Netw., vol. 7, no. 7, pp. 6348-6359, 2020.

[345] L. U. Khan, W. Saad, Z. Han, E. Hossain, and C. S. Hong, "Federated learning for internet of things: Recent advances, taxonomy, and open challenges," arXiv preprint arXiv:2009.13012, 2020.

[346] Z. Du, C. Wu, T. Yoshinaga, K.-L. A. Yau, Y. Ji, and J. Li, "Federated learning for vehicular Internet of things: Recent advances and open issues," IEEE Open J. Comput. Soc, vol. 1, pp. 45-61, 2020.

[347] D. C. Nguyen, M. Ding, P. N. Pathirana, A. Seneviratne, J. Li, and H. V. Poor, "Federated learning for Internet of Things: A comprehensive survey," arXiv preprint arXiv:2104.07914, 2021.

[348] E. Denton, W. Zaremba, J. Bruna, Y. LeCun, and R. Fergus, "Exploiting linear structure within convolutional networks for efficient evaluation," arXiv preprint arXiv:1404.0736, 2014.

[349] N. D. Lane, S. Bhattacharya, P. Georgiev, C. Forlivesi, and F. Kawsar, "An early resource characterization of deep learning on wearables, smartphones and Internet-of-things devices," in Proc. Iint. workshop Internet things Appl., 2015, pp. 7-12.

[350] M. Jaderberg, A. Vedaldi, and A. Zisserman, "Speeding up convolutional neural networks with low rank expansions," arXiv preprint arXiv:1405.3866, 2014

[351] S. Han, J. Pool, J. Tran, and W. J. Dally, "Learning both weights and connections for efficient neural networks," arXiv preprint arXiv:1506.02626, 2015.

[352] Y. Guo, A. Yao, and Y. Chen, "Dynamic network surgery for efficient DNNs," arXiv preprint arXiv:1608.04493, 2016.

[353] Y. LeCun, J. S. Denker, S. A. Solla, R. E. Howard, and L. D. Jackel, "Optimal brain damage," in Proc. Neural Inform. Process. Syst. (NIPS), vol. 2, 1989, pp. 598-605.

[354] V. Vanhoucke, A. Senior, and M. Z. Mao, "Improving the speed of neural networks on CPUs," in Deep Learning and Unsupervised Feature Learning Workshop (NIPS Conference), 2011.

[355] Y. Gong, L. Liu, M. Yang, and L. Bourdev, "Compressing deep convolutional networks using vector quantization," arXiv preprint arXiv:1412.6115, 2014.

[356] J. Wu, C. Leng, Y. Wang, Q. Hu, and J. Cheng, "Quantized convolutional neural networks for mobile devices," in Proc. IEEE Conf. Comput. Vis. Pattern Recognit. (CVPR), 2016, pp. 4820-4828.

[357] M. Courbariaux, Y. Bengio, and J.-P. David, "Binaryconnect: Training deep neural networks with binary weights during propagations," arXiv preprint arXiv:1511.00363, 2015.

[358] W. Tang, G. Hua, and L. Wang, "How to train a compact binary neural network with high accuracy?" in Proc. AAAI Conf. Artif. Intell., vol. 31, no. $1,2017$.

[359] S. Han, H. Mao, and W. J. Dally, "Deep compression: Compressing deep neural networks with pruning, trained quantization and huffman coding," arXiv preprint arXiv:1510.00149, 2015.

[360] M. Sandler, A. Howard, M. Zhu, A. Zhmoginov, and L.-C. Chen, "Mobilenetv2: Inverted residuals and linear bottlenecks," in Proc. IEEE Conf. Comput. Vis. Pattern Recognit. (CVPR), 2018, pp. 4510-4520.

[361] F. N. Iandola, S. Han, M. W. Moskewicz, K. Ashraf, W. J. Dally, and K. Keutzer, "SqueezeNet: AlexNet-level accuracy with 50x fewer parameters andi 0.5 MB model size," arXiv preprint arXiv:1602.07360, 2016.

[362] J. Lin, W.-M. Chen, Y. Lin, J. Cohn, C. Gan, and S. Han, "Mcunet: Tiny deep learning on IoT devices," arXiv preprint arXiv:2007.10319, 2020.

[363] G. T. 22.822, "Study on using satellite access in 5G" 2018. [Online]. Available: http://www.3gpp.org/ftp//Specs/archive/22_series/ 22.822/22822-g00.zip

[364] O. Liberg, S. E. Löwenmark, S. Euler, B. Hofström, T. Khan, X. Lin, and J. Sedin, "Narrowband Internet of Things for non-terrestrial networks," IEEE Commun. Stand. Mag., vol. 4, no. 4, pp. 49-55, 2020.

[365] G. Geraci, A. Garcia-Rodriguez, M. M. Azari, A. Lozano, M. Mezzavilla, S. Chatzinotas, Y. Chen, S. Rangan, and M. Di Renzo, "What will the future of UAV cellular communications be? a flight from $5 \mathrm{G}$ to 6G," arXiv preprint arXiv:2105.04842, 2021.

[366] E. Vinogradov, H. Sallouha, S. De Bast, M. M. Azari, and S. Pollin, "Tutorial on UAV: A blue sky view on wireless communication," arXiv preprint arXiv:1901.02306, 2019. 
[367] M. M. Azari, F. Rosas, K.-C. Chen, and S. Pollin, "Ultra reliable UAV communication using altitude and cooperation diversity," IEEE Trans. Commun., vol. 66, no. 1, pp. 330-344, 2017.

[368] C. Zhan and H. Lai, "Energy minimization in Internet-of-Things system based on rotary-wing UAV," IEEE Wireless Commun. Lett., vol. 8, no. 5, pp. 1341-1344, 2019

[369] S. Fu, Y. Tang, Y. Wu, N. Zhang, H. Gu, C. Chen, and M. Liu, "Energy-efficient UAV enabled data collection via wireless charging: A reinforcement learning approach," IEEE Internet Things J., 2021.

[370] J. Li, H. Zhao, H. Wang, F. Gu, J. Wei, H. Yin, and B. Ren, "Joint optimization on trajectory, altitude, velocity, and link scheduling for minimum mission time in UAV-aided data collection," IEEE Internet Things J., vol. 7, no. 2, pp. 1464-1475, 2020.

[371] S. Fu, Y. Tang, N. Zhang, L. Zhao, S. Wu, and X. Jian, "Joint unmanned aerial vehicle (UAV) deployment and power control for Internet of Things networks," IEEE Trans. Veh. Technol., vol. 69, no. 4, pp. 43674378, 2020.

[372] O. A. Saraereh, A. Alsaraira, I. Khan, and P. Uthansakul, "Performance evaluation of UAV-enabled LoRa networks for disaster management applications," Sensors, vol. 20, no. 8, p. 2396, 2020.

[373] H. Bayerlein, M. Theile, M. Caccamo, and D. Gesbert, "UAV path planning for wireless data harvesting: A deep reinforcement learning approach," arXiv preprint arXiv:2007.00544, 2020.

[374] Y. Cao, L. Zhang, and Y.-C. Liang, "Deep reinforcement learning for channel and power allocation in UAV-enabled IoT systems," in Proc. IEEE Global Commun. Conf. (GLOBECOM), 2019, pp. 1-6.

[375] W. Alshrafi, U. Engel, and T. Bertuch, "Compact controlled reception pattern antenna for interference mitigation tasks of global navigation satellite system receivers," IET Microwaves, Antennas \& Propagation, vol. 9, no. 6, pp. 593-601, 2014.

[376] G. Han et al., "A survey on mobile anchor node assisted localization in wireless sensor networks," IEEE Commun. Surveys Tuts., vol. 18, no. 3, pp. 2220-2243, 2016

[377] S. Gezici, Z. Tian, G. B. Giannakis, H. Kobayashi, A. F. Molisch H. V. Poor, and Z. Sahinoglu, "Localization via ultra-wideband radios: a look at positioning aspects for future sensor networks," IEEE Signal Process. Mag., vol. 22, no. 4, pp. 70-84, 2005.

[378] A. Zanella, "Best practice in RSS measurements and ranging," IEEE Commun. Surveys Tuts., vol. 18, no. 4, pp. 2662-2686, 2016.

[379] A. Al-Hourani, S. Kandeepan, and A. Jamalipour, "Modeling air-toground path loss for low altitude platforms in urban environments," in Proc. IEEE Global Commun. Conf. (GLOBECOM), 2014, pp. 28982904.

[380] M. M. Azari, H. Sallouha, A. Chiumento, S. Rajendran, E. Vinogradov, and S. Pollin, "Key technologies and system trade-offs for detection and localization of amateur drones," IEEE Commun. Mag., vol. 56, no. 1, pp. 51-57, 2018.

[381] H. Sallouha, M. M. Azari, A. Chiumento, and S. Pollin, "Aerial anchors positioning for reliable RSS-based outdoor localization in urban environments," IEEE Wireless Commun. Lett., 2018.

[382] H. Sallouha, M. M. Azari, and S. Pollin, "Energy-constrained UAV trajectory design for ground node localization," in Proc. IEEE Global Commun. Conf. (GLOBECOM), 2018, pp. 1-7.

[383] J. Park, H. Lee, S. Eom, and I. Lee, "UAV-aided wireless powered communication networks: Trajectory optimization and resource allocation for minimum throughput maximization," IEEE Access, vol. 7, pp. 134 978-134991, 2019.

[384] Q. Wu, Y. Zeng, and R. Zhang, "Joint trajectory and communication design for multi-UAV enabled wireless networks," IEEE Trans. Wireless Commun., vol. 17, no. 3, pp. 2109-2121, 2018.

[385] W. Feng, J. Tang, Y. Yu, J. Song, N. Zhao, G. Chen, K.-K. Wong, and J. Chambers, "UAV-enabled SWIPT in IoT networks for emergency communications," IEEE Wireless Commun., vol. 27, no. 5, pp. 140$147,2020$.

[386] C. Jeong and S. H. Chae, "Simultaneous wireless information and power transfer for multi-user UAV-enabled IoT networks," IEEE Internet Things J., 2020.

[387] F. Huang, J. Chen, H. Wang, G. Ding, Y. Gong, and Y. Yang, "MultipleUAV-assisted SWIPT in Internet of Things: User association and power allocation," IEEE Access, vol. 7, pp. 124 244-124 255, 2019.

[388] J.-M. Kang and C.-J. Chun, "Joint trajectory design, Tx power allocation, and Rx power splitting for UAV-enabled multicasting SWIPT systems," IEEE Systems Journal, vol. 14, no. 3, pp. 3740-3743, 2020.

[389] M. M. Azari, F. Rosas, and S. Pollin, "Cellular connectivity for UAVs: Network modeling, performance analysis, and design guidelines," IEEE Trans. Wireless Commun., vol. 18, no. 7, pp. 3366-3381, 2019.
[390] "3rd generation partnership project; technical specification group radio access network; study on enhanced lte support for aerial vehicles (release 15)," Tech. Rep., Jan. 2018. [Online]. Available: www.3gpp.org/dynareport/36777.htm

[391] M. M. Azari, F. Rosas, A. Chiumento, and S. Pollin, "Coexistence of terrestrial and aerial users in cellular networks," in Proc. IEEE Global Commun. Conf. Workshops (GC Workshops), 2017, pp. 1-6.

[392] M. M. Azari, G. Geraci, A. Garcia-Rodriguez, and S. Pollin, "UAVto-UAV communications in cellular networks," IEEE Trans. Wireless Commun., vol. 19, no. 9, pp. 6130-6144, 2020.

[393] B. Soret, I. Leyva-Mayorga, S. Cioni, and P. Popovski, "5G satellite networks for IoT: offloading and backhauling," arXiv preprint arXiv:2011.05202, 2020.

[394] Y. Shi, Y. Xia, and Y. Ga, "Joint gateway selection and resource allocation for cross-tier communication in space-air-ground integrated IoT networks," IEEE Access, 2020.

[395] H. Dai, H. Bian, C. Li, and B. Wang, "UAV-aided wireless communication design with energy constraint in space-air-ground integrated green IoT networks," IEEE Access, vol. 8, pp. 86 251-86 261, 2020.

[396] B. Zhao, J. Liu, Z. Wei, and I. You, "A deep reinforcement learning based approach for energy-efficient channel allocation in satellite Internet of things," IEEE Access, vol. 8, pp. 62 197-62 206, 2020

[397] G. Cui, X. Li, L. Xu, and W. Wang, "Latency and energy optimization for mec enhanced SAT-IoT networks," IEEE Access, vol. 8, pp. 5591555 926, 2020.

[398] A. Azari et al., "Grant-free radio access for short-packet communications over 5G networks," in Proc. IEEE Global Commun. Conf. (GLOBECOM), 2017.

[399] H. Tabassum et al., "Non-orthogonal multiple access (NOMA) in cellular uplink and downlink: Challenges and enabling techniques," arXiv preprint arXiv:1608.05783, 2016.

[400] Z. Ding et al., "A survey on non-orthogonal multiple access for 5G networks: Research challenges and future trends," IEEE J. Sel. Areas Commun., vol. 35, no. 10, pp. 2181-2195, July 2017.

[401] O. L. A. López, H. Alves, R. D. Souza, S. Montejo-Sánchez, E. M. G. Fernández, and M. Latva-aho, "Massive wireless energy transfer: Enabling sustainable IoT towards 6G era," arXiv preprint arXiv:1912.05322, 2019.

[402] J. Ding, D. Qu, H. Jiang, and T. Jiang, "Success probability of grantfree random access with massive MIMO," IEEE Internet Things $J$, vol. 6, no. 1, pp. 506-516, 2019.

[403] X. Jiang et al., "Low-latency networking: Where latency lurks and how to tame it," Proceedings of the IEEE, vol. 107, no. 2, pp. 280-306, Feb 2019.

[404] R. Abbas et al., "A novel analytical framework for massive Grant-Free NOMA," IEEE Trans. Commun., vol. 67, no. 3, pp. 2436-2449, 2019.

[405] N. Ye et al., "Deep learning aided grant-free NOMA towards reliable low-latency access in tactile Internet of things," IEEE Trans. Ind. Informat., 2019.

[406] R1-1808304, "Discussion on the reliability enhancement for grant-free transmission," Tech. Rep., Aug. 2018, 3GPP TSG-RAN1 Meeting 94, Gothenburg, Sweden.

[407] H. Jiang et al., "Distributed layered grant-free non-orthogonal multiple access for massive MTC," in Proc. IEEE Int. Symp. Pers. Indoor Mobile Radio-commun. (PIMRC), 2018.

[408] A. Azari, M. Ozger, and C. Cavdar, "Risk-aware resource allocation for URLLC: Challenges and strategies with machine learning," IEEE Commun. Mag, vol. 57, no. 3, pp. 42-48, March 2019.

[409] Z. Li et al., "2D time-frequency interference modeling using stochastic geometry for performance evaluation in low-power wide-area networks," arXiv preprint arXiv:1606.04791, May 2016.

[410] Y. Zhang, K. Peng, Z. Chen, and J. Song, "SIC vs. JD: Uplink NOMA techniques for M2M random access," in Proc. IEEE Int. Commun. Conf. (ICC), May 2017.

[411] M. Masoudi, A. Azari, E. A. Yavuz, and C. Cavdar, "Grant-free radio access IoT networks: Scalability analysis in coexistence scenarios," in Proc. IEEE Int. Commun. Conf. (ICC), 2018, pp. 1-7.

[412] R. Abbas, M. Shirvanimoghaddam, Y. Li, and B. Vucetic, "Grant-free massive NOMA: Outage probability and throughput," arXiv preprint arXiv:1707.07401, 2017

[413] D. D. Wcntzloff, A. Alghaihab, and J. Im, "Ultra-low power receivers for IoT applications: A review," in Proc. IEEE Custom Integr. Circuits Conf., 2020, pp. 1-8.

[414] H. Khodr, N. Kouzayha, M. Abdallah, J. Costantine, and Z. Dawy, "Energy efficient IoT sensor with RF wake-up and addressing capability," IEEE sensors lett., vol. 1, no. 6, pp. 1-4, 2017. 
[415] T. Sanislav, G. D. Mois, S. Zeadally, and S. C. Folea, "Energy harvesting techniques for Internet of things (IoT)," IEEE Access, vol. 9, pp. 39 530-39549, 2021.

[416] A. Haridas, V. S. Rao, R. V. Prasad, and C. Sarkar, "Opportunities and challenges in using energy-harvesting for NB-IoT," ACM Sigbed Review, vol. 15, no. 5, pp. 7-13, 2018.

[417] Y. Liu, Y. Deng, M. Elkashlan, A. Nallanathan, J. Yuan, and R. K. Mallik, "RACH in self-powered NB-IoT networks: Energy availability and performance evaluation," arXiv preprint arXiv:2011.11723, 2020.

[418] M. Eshaghi and R. Rashidzadeh, "An energy harvesting solution for IoT devices in 5G networks," in Proc. IEEE Canadian Conf. Electr. Comput. Eng. (CCECE). IEEE, 2020, pp. 1-4.

[419] N. Kaina, M. Dupré, G. Lerosey, and M. Fink, "Shaping complex microwave fields in reverberating media with binary tunable metasurfaces," Scientific reports, vol. 4, no. 1, pp. 1-8, 2014.

[420] Z. Zhao, M. Peng, Z. Ding, W. Wang, and H.-H. Chen, "Denoiseand-forward network coding for two-way relay MIMO systems," IEEE Trans. Veh. Technol., vol. 63, no. 2, pp. 775-788, 2013.

[421] G. Liu, F. R. Yu, H. Ji, V. C. Leung, and X. Li, "In-band fullduplex relaying: A survey, research issues and challenges," IEEE Communications Surveys \& Tutorials, vol. 17, no. 2, pp. 500-524, 2015.

[422] G. Bauch and J. S. Malik, "Cyclic delay diversity with bit-interleaved coded modulation in orthogonal frequency division multiple access," IEEE Trans. Wireless Comm., vol. 5, no. 8, pp. 2092-2100, 2006.

[423] F. Fang, H. Zhang, J. Cheng, S. Roy, and V. C. Leung, "Joint user scheduling and power allocation optimization for energy-efficient noma systems with imperfect csi," IEEE J. Sel. Areas Commun., vol. 35, no. 12, pp. 2874-2885, 2017.

[424] D.-T. Do, M. Vaezi, and T.-L. Nguyen, "Wireless powered cooperative relaying using NOMA with imperfect CSI," in Proc. IEEE Global Commun. Conf. Workshops (GC Workshops). IEEE, 2018, pp. 1-6.

[425] S. Arzykulov, T. A. Tsiftsis, G. Nauryzbayev, and M. Abdallah, "Outage performance of cooperative underlay CR-NOMA with imperfect CSI," IEEE Commun. Lett., vol. 23, no. 1, pp. 176-179, 2018.

[426] Y. Qi, X. Zhang, and M. Vaezi, "Over-the-air implementation of NOMA: New experiments, existing literature and future directions," submitted for publication, 2021.

[427] S. Shao, P. Hailes, T.-Y. Wang, J.-Y. Wu, R. G. Maunder, B. M. AlHashimi, and L. Hanzo, "Survey of turbo, LDPC, and polar decoder ASIC implementations," IEEE Communications Surveys \& Tutorials, vol. 21, no. 3, pp. 2309-2333, 2019.

[428] B. Zhou and W. Saad, "Joint status sampling and updating for minimizing age of information in the Internet of Things," IEEE Trans. Commun., vol. 67, no. 11, pp. 7468-7482, 2019.

[429] B. Wang, S. Feng, and J. Yang, "When to preempt? age of information minimization under link capacity constraint," IEEE J. Commun. Netw., vol. 21, no. 3, pp. 220-232, 2019.

[430] B. Zhou, W. Saad, M. Bennis, and P. Popovski, "Risk-aware optimization of age of information in the Internet of Things," in Proc. IEEE Int. Commun. Conf. (ICC), 2020, pp. 1-6.

[431] F. Farha, H. Ning, K. Ali, L. Chen, and C. Nugent, "SRAM-PUF-based entities authentication scheme for resource-constrained IoT devices," IEEE Int. of Things, vol. 8, no. 7, pp. 5904-5913, 2021.

[432] J. Yoo, D. Kim, H. Park, M. Shim, C. Lee, and C. Kim, "Physically unclonable function using ring oscillator collapse in $0.5 \mathrm{~V}$ nearthreshold voltage for low-power Internet of Things," in Proc. IEEE Int. Conf. on Consum. Electron. Asia (ICCE-Asia), 2018, pp. 206-212.

\section{Appendix A: List of AbBREviation}

A complete list of abbreviation can be found in the table below.

\section{GLOSSARY}

$1 \mathrm{G}$ first-generation (cellular networks)

$2 \mathrm{G}$ second-generation

3D three-dimensional

3G third-generation

3GPP 3rd generation partnership project

$4 \mathrm{G}$ fourth-generation
$5 \mathrm{G} \quad$ fifth-generation

$6 \mathrm{G}$ sixth-generation

ACMA asynchronous coded multiple access

ADC analog-to-digital converter

AE autoencoder

AFC analog fountain code

AI artificial intelligence

AKA authentication and key agreement

ANN artificial neural network

AoI age of information

AP access point

B5G beyond $5 \mathrm{G}$

$\mathrm{BCH}$ Bose-Chaudhuri-Hocquenghem

BLE Bluetooth low-energy

BLER block error rate

BP belief propagation

BS base station

BW bandwidth

CAGR compound annual growth rate

CDD cyclic delay diversity

CDMA code division multiple access

CNN convolutional neural network

CQI channel quality indicator

CRC cyclic redundancy check

CSI channel state information

CSS chirp spread spectrum

D2D device-to-device

DDoS distributed denial-of-service

DFT discrete Fourier transform

DL deep learning

DNN deep neural network

DNS domain name system

DPC dirty paper coding

DRL deep reinforcement learning

E2E end-to-end

eMBB enhanced mobile broadband

FL federated learning

GAN generative adversarial network

GFA grant-free access

gNB gNodeB

GPS global positioning system

HARQ hybrid automatic repeat request

IDMA interleave division multiple access

IDS intrusion detection system

IGMA interleave-grid multiple access

IIoT industrial IoT

IMT international mobile telecommunications 


\begin{tabular}{|c|c|c|c|}
\hline IoT & Internet of things & PER & packet error rate \\
\hline ISD & inter-site distance & PHY & physical layer \\
\hline ISM & industrial safety medical & PUCCH & physical uplink control channel \\
\hline \multirow[t]{2}{*}{ ITU } & International Telecommunications & PUF & physically unclonable function \\
\hline & Union & PUSCH & physical uplink shared channel \\
\hline KPI & key performance indicator & QoS & quality of service \\
\hline LCRS & low code rate spreading & $\mathrm{RACH}$ & random access channel \\
\hline LDPC & low-density parity-check & $\mathrm{RB}$ & resource block \\
\hline LDS & low-density signature & RBM & restricted Boltzmann machine \\
\hline LEO & low earth orbit & RDMA & repetition division multiple access \\
\hline LLR & log-likelihood ratio & ReLU & rectified linear units \\
\hline LoRa & long range & RF & radio frequency \\
\hline LoRaWAN & LoRa wide area network & RFID & radio-frequency identification \\
\hline LOS & line-of-sight & RL & reinforcement learning \\
\hline LPWA & low-power wide-area & RNN & recurrent neural network \\
\hline LTE & Long Term Evolution & RSMA & resource spread multiple access \\
\hline LTE-A & LTE-Advanced & RSS & received signal strength \\
\hline \multirow[t]{2}{*}{ LTE-M } & LTE-MTC & & \\
\hline & & $\mathrm{SC}$ & successive cancellation \\
\hline MAC & media access control & SC-FDMA & single-carrier FDMA \\
\hline MIMO & multiple-input multiple-output & SC-SIC & superposition coding-successive in- \\
\hline MISO & multiple-input single-output & & terference cancellation \\
\hline ML & machine learning & SCMA & sparse code multiple access \\
\hline \multirow[t]{2}{*}{ mMTC } & massive machine type of communi- & SDN & software defined networking \\
\hline & cation & SF & spreading factor \\
\hline mmWave & millimeter-wave & $\mathrm{SIC}$ & successive interference cancellation \\
\hline MPA & message passing algorithm & SISO & single-input and single-output \\
\hline \multirow[t]{2}{*}{ MUSA } & multi-user shared access & SNR & signal-to-noise ratio \\
\hline & & SQL & structured query language \\
\hline NB-IoT & narrowband IoT & SRAM & static random-access memory \\
\hline NOCA & non-orthogonal coded access & SVD & singular value decomposition \\
\hline NOMA & non-orthogonal multiple access & SVM & support vector machine \\
\hline \multirow[t]{2}{*}{$\mathrm{NPBCH}$} & narrowband physical broadcast chan- & & \\
\hline & nel & TBCC & tail-biting convolutional code \\
\hline \multirow[t]{2}{*}{$\mathrm{NPDCCH}$} & narrowband physical downlink con- & $\mathrm{THz}$ & terahertz \\
\hline & trol channel & TLS & transport layer security \\
\hline \multirow[t]{2}{*}{$\mathrm{NPDSCH}$} & narrowband physical downlink & TRP & transmission reception point \\
\hline & shared channel & TTI & transmission time interval \\
\hline \multirow[t]{2}{*}{ NPRACH } & narrowband physical random access & & \\
\hline & channel & UAV & unmanned aerial vehicle \\
\hline \multirow[t]{2}{*}{ NPSS } & narrowband primary synchronization & UE & user equipment \\
\hline & signal & UHD & ultra-high definition \\
\hline NPUSCH & $\begin{array}{l}\text { narrowband physical uplink shared } \\
\text { channel }\end{array}$ & URLLC & $\begin{array}{l}\text { ultra-reliable low-latency communi- } \\
\text { cations }\end{array}$ \\
\hline NR & new radio & UWB & ultra wideband \\
\hline NRS & narrowband reference signal & & \\
\hline \multirow[t]{2}{*}{ NTN } & non-terrestrial network & WPT & wireless power transfer \\
\hline & & WSN & wireless sensor network \\
\hline OFDM & $\begin{array}{l}\text { orthogonal frequency-division multi- } \\
\text { plexing }\end{array}$ & & \\
\hline OSD & ordered statistics decoder & & \\
\hline $\mathrm{PDCCH}$ & physical downlink control channel & & \\
\hline PDMA & pattern division multiple access & & \\
\hline
\end{tabular}

